NASA Technical Memorandum 100911

AIAA-88-3567

\title{
A Diagonally Inverted LU Implicit Multigrid Scheme
}

Jeffrey W. Yokota

Sverdrup Technology, Inc.

NASA Lewis Research Center Group

Cleveland, Ohio

David A. Caughey

Cornell University

Ithaca, New York

and

Rodrick V. Chima

National Aeronautics and Space Administration

Lewis Research Center

Cleveland, Ohio

Prepared for the

First National Fluid Dynamics Congress

cosponsored by the AIAA, ASME, ASCE, SIAM, and APS

Cincinnati, Ohio, July 24-28, 1988

\section{N/SA}




\title{
A Diagonally Inverted LU Implicit Multigrid Scheme
}

\author{
Jeffrey W. Yokota \\ Sverdrup Technology, Inc. \\ NASA Lewis Research Center Group \\ Cleveland, Ohio \\ David A. Caughey \\ Sibley School of Mechanical And Aerospace Engineering \\ Cornell University \\ Ithaca, New York \\ Rodrick V. Chima \\ NASA Lewis Research Center \\ Cleveland, Ohio
}

\begin{abstract}
A new Diagonally Inverted LU Implicit scheme is developed within the framework of the multigrid method for the three-dimensional unsteady Euler equations. The matrix systems that are to be inverted in the LU scheme are treated by local diagonalizing transformations that decouple them into systems of scalar equations. Unlike the Diagonalized ADI method, the time accuracy of the LU scheme is not reduced since the diagonalizing procedure does not destroy time conservation. Even more importantly, this diagonalization significantly reduces the computational effort required to solve the $L U$ approximation and therefore transforms it into a more efficient method of numerically solving the three-dimensional Euler equations.
\end{abstract}

\section{Introduction}

Increasingly, attention is being focused upon implicit schemes as the need to develop more efficient numerical schemes becomes apparent. An implicit approximation is an attractive means of increasing the efficiency of the timemarching technique since an implicit scheme allows one to take much larger time steps than are normally allowed in most explicit methods. The drawback of most implicit schemes is that they are computationally more expensive, per time step, than explicit methods and thus interest is focused upon decreasing the computational effort required to solve the implicit approximation. Examples of schemes that address this issue are Chaussee and Pulliam's Diagonalized $A D I^{1}$, Obayashi and Fujii's LU-ADI ${ }^{2}$ (based on the unsteady Euler and Navier-Stokes equations) and Yoon and Jameson's LU-SSOR ${ }^{3}$ (for the steady equations).

The three-dimensional unsteady Euler equations can be discretized into an implicit approximation that, when written in delta-form, produces a large block banded matrix system that is impractical to solve without first being approximately factored. In the present work, the LU Implicit Multigrid algorithm developed by Yokota and Caughey ${ }^{4}$ for the three-dimensional unsteady Euler equations has been made more efficient through a local diagonalizing procedure that reduces the computational effort required to solve the LU approximation. This new Diagonally Inverted $L U$ scheme is related to the $L U$ factorization first proposed by Jameson and Turke ${ }^{5}$ and applied to the two-dimensional Euler equations by Buratynski and Caughey ${ }^{6}$. The LU factorization produces two block triangular operators (one upper and one lower) which, through back substitution, can be solved by effectively explicit sweeps that require matrix inversions at every mesh cell in the domain. The efficiency of the LU scheme can be significantly increased by reducing the computational costs associated with these matrix inversions. This reduction can be achieved by a local diagonalizing transformation that decouples the matrix systems into scalar equations. Time conservation and stability are not altered by the decoupling since the LU scheme's differencing operators are not affected by the diagonalizing transformations. This result is in contrast to the Diagonalized ADI method, which is produced at the expense of time accuracy. Thus the solution of the Diagonally Inverted $L U$ scheme requires less computational effort than the original LU scheme and remains both unconditionally stable and time accurate. The Euler equations are numerically transformed to a generalized coordinate system and then discretized into a finite volume approximation that is supplemented with an adaptive blend of second and fourth difference artificial dissipation. Numerical results show that the Diagonally Inverted LU scheme requires less than $1 / 4$ the CPU time the original LU scheme would otherwise need. In addition, the diagonally Inverted LU scheme is developed within the framework of the multigrid method to accelerate the convergence rate for steady state calculations. Results for turbomachinery flows are presented to illustrate the $\mathrm{Di}$ agonally Inverted LU scheme's ability to calculate Euler flows and the convergence acceleration produced by the multigrid method.

\section{Analysis}

The unsteady three-dimensional Euler equations are written in divergence form and then transformed from the Cartesian coordinate system $(x, y, z)$ to the generalized system $(\xi, \eta, \varsigma)$. The resulting equations can be written:

$$
\frac{\partial \vec{W}}{\partial t}+\frac{\partial \vec{F}}{\partial \xi}+\frac{\partial \vec{G}}{\partial \eta}+\frac{\partial \vec{H}}{\partial \zeta}=0
$$

with

$$
\begin{gathered}
\vec{W}=\left(\begin{array}{c}
\rho D \\
\rho D u \\
\rho D v \\
\rho D w \\
\rho E
\end{array}\right) \quad \vec{F}=\left(\begin{array}{c}
\rho D U \\
\rho D U u+P\left(y_{\eta} z_{\varsigma}-y_{\varsigma} z_{\eta}\right) \\
\rho D U v+P\left(x_{\varsigma} z_{\eta}-x_{\eta} z_{\zeta}\right) \\
\rho D U w+P\left(x_{\eta} y_{\varsigma}-x_{\varsigma} y_{\eta}\right) \\
D U(E+P)
\end{array}\right) \\
\vec{G}=\left(\begin{array}{c}
\rho D V u+P\left(y_{\varsigma} z_{\xi}-y_{\xi} z_{\xi}\right) \\
\rho D V v+P\left(x_{\xi} z_{\varsigma}-x_{\xi} z_{\xi}\right) \\
\rho D V w+P\left(x_{\varsigma} y_{\xi}-x_{\xi} y_{\varsigma}\right) \\
D V(E+P)
\end{array}\right)
\end{gathered}
$$




$$
\vec{H}=\left(\begin{array}{c}
\rho D W \\
\rho D W u+P\left(y_{\xi} z_{\eta}-y_{\eta} z_{\xi}\right) \\
\rho D W v+P\left(x_{\eta} z_{\xi}-x_{\xi} z_{\eta}\right) \\
\rho D W w+P\left(x_{\xi} y_{\eta}-x_{\eta} y_{\xi}\right) \\
D W(E+P)
\end{array}\right)
$$

where $\rho$ and $P$ are the fluid density and pressure, $(u, v, w)$ are the Cartesian velocity components and $E$ is the total energy per unit volume. The total energy and pressure of a calorically perfect gas are related through the equation of state:

$$
P=(\gamma-1)\left(E-\rho \frac{\vec{v} \cdot \vec{v}}{2}\right)
$$

The Jacobians of the coordinate transformation are as follows:

$$
\begin{gathered}
J=\left(\begin{array}{lll}
x_{\xi} & x_{\eta} & x_{\xi} \\
y_{\xi} & y_{\eta} & y_{\xi} \\
z_{\xi} & z_{\eta} & z_{\xi}
\end{array}\right) \\
J^{-1}=\left(\begin{array}{lll}
\xi_{x} & \xi_{y} & \xi_{z} \\
\eta_{x} & \eta_{y} & \eta_{z} \\
\zeta_{x} & \zeta_{y} & \zeta_{z}
\end{array}\right) \\
=\frac{1}{D}\left(\begin{array}{lll}
y_{\eta} z_{\zeta}-y_{\zeta} z_{\eta} & x_{\zeta} z_{\eta}-x_{\eta} z_{\zeta} & x_{\eta} y_{\zeta}-x_{\xi} y_{\eta} \\
y_{\zeta} z_{\xi}-y_{\xi} z_{\zeta} & x_{\xi} z_{\zeta}-x_{\xi} z_{\xi} & x_{\xi} y_{\xi}-x_{\xi} y_{\zeta} \\
y_{\xi} z_{\eta}-y_{\eta} z_{\xi} & x_{\eta} z_{\xi}-x_{\xi} z_{\eta} & x_{\xi} y_{\eta}-x_{\eta} y_{\xi}
\end{array}\right)
\end{gathered}
$$

where $D$ is the determinant of the matrix $J$ and the contravariant velocities are defined as:

$$
\left(\begin{array}{c}
U \\
V \\
W
\end{array}\right)=J^{-1}\left(\begin{array}{c}
u \\
v \\
w
\end{array}\right)
$$

The transformed Euler equations are discretized by a finite volume formulation that approximates the spatial differences as a net flux across the faces of each mesh cell. Global conservation and the admission of possible uniform flow solutions are insured by evaluating the flux vectors on the faces of the boundary-conforming mesh cells. This procedure requires that both the flow variables and the fluxembedded geometric quantities be defined on the faces of the mesh cells during the flux evaluations although it is the cell-averaged flow variables that are calculated during the time and spatial marching. The geometric quantities are evaluated directly on the cell faces while the flow variables are averaged over values found in adjacent cells. The unsteady equations can be discretized into an implicit approximation that when written in a linearized delta form, produces a numerical scheme whose steady state solutions are independent of the time step size used in the time marching. The delta form, produced by linearizing the changes in the flux vectors through a Taylor series expansion about a time level $n$, can be written as follows:

$$
\begin{gathered}
{\left[I+\mu \Delta t\left(\delta_{\xi} A+\delta_{\eta} B+\delta_{\xi} C\right)\right] \Delta \vec{W}_{i j k}^{n}=} \\
-\Delta t\left(\bar{\delta}_{\xi} \vec{F}+\bar{\delta}_{\eta} \vec{G}+\bar{\delta}_{\xi} \vec{H}\right)_{i j k}^{n}
\end{gathered}
$$

where

$$
\Delta \vec{W}^{n}=\vec{W}^{n+1}-\vec{W}^{n}
$$

and $\Delta t$ is the time step size; $0 \leq \mu \leq 1$ is a parameter governing the degree of implicitness; $\delta$ and $\delta$ are cell- and face-centered central differences; $I$ is the identity matrix; and $A, B$, and $C$ are the flux Jacobian matrices relative to the vectors $\vec{F}, \vec{G}$, and $\vec{H}$.

\section{Artificial Dissipation}

The finite volume formulation reduces to a central difference approximation on a uniform grid and therefore requires the addition of explicit artificial dissipation terms to suppress possible odd and even point oscillations and shock overshoots. Following the works of Jameson ${ }^{7}$ and Pulliam ${ }^{8}$, fourth difference terms are added throughout the flow field to prevent odd-even decoupling while second difference terms are used to stablize the flow calculation near shocks.

The conservatively added dissipative term is an adaptive nonlinear blend of second and fourth differences that acts to turn on the second and turn off the fourth difference terms near a shock. The dissipative terms added are of the form:

$$
\vec{T}_{i}=-\vec{\delta}_{\xi} \kappa^{(2)} D \bar{\delta}_{\xi} \frac{1}{D} \vec{W}_{i}+\widetilde{\delta}_{\xi} \kappa^{(4)} D \bar{\delta}_{\xi \xi \xi} \frac{1}{D} \vec{W}_{i}
$$

where a shock sensing term:

$$
\nu_{i}=\frac{\left|P_{i+1}-2 P_{i}+P_{i-1}\right|}{P_{i+1}+2 P_{i}+P_{i-1}}
$$

is used to define the nonlinear scaling factors:

$$
\begin{gathered}
\kappa_{i+\frac{1}{2}}^{(2)}=\frac{\chi^{(2)}}{\Delta t_{i+\frac{1}{2}}^{\prime}} \max \left(\nu_{i+2}, \nu_{i+1}, \nu_{i}, \nu_{i-1}\right) \\
\kappa_{i+\frac{1}{2}}^{(4)}=\max \left(0, \frac{\chi^{(4)}}{\Delta t_{i+\frac{1}{2}}^{\prime}}-\kappa_{i+\frac{1}{2}}^{(2)}\right)
\end{gathered}
$$

where $\chi^{(2)}$ and $\chi^{(4)}$ are scalar constants and $\Delta t^{\prime}$ is a onedimensional unit Courant number time step scaling factor.

\section{LU Factorization}

The block-banded implict delta operator is factored into two block triangular operators to circumvent the large amounts of CPU time and temporary storage needed to solve the unfactored implicit approximation. The LU factorization, which is based on one-sided, implicit, spatial differences, can be written as follows:

$$
\begin{gathered}
{\left[I+\mu \Delta t\left(\delta_{\xi}^{-} A_{1}+\delta_{\eta}^{-} B_{1}+\delta_{\zeta}^{-} C_{1}\right)\right]} \\
{\left[I+\mu \Delta t\left(\delta_{\xi}^{+} A_{2}+\delta_{\eta}^{+} B_{2}+\delta_{\zeta}^{+} C_{2}\right)\right] \Delta \vec{W}_{i j k}^{n}=} \\
-\Delta t\left(\delta_{\xi} \vec{F}+\vec{\delta}_{\eta} \vec{G}+\delta_{\zeta} \vec{H}+\vec{T}\right)_{i j k}^{n}
\end{gathered}
$$

where $\delta^{+}$and $\delta^{-}$are cell-centered forward and backward first differences and the flux Jacobian matrices are split and reconstructed as:

$$
A_{1}=\frac{(A+\beta|A| I)}{2} \quad A_{2}=\frac{(A-\beta|A| I)}{2}
$$


where

$$
|A|=\max \left(\left|\lambda_{A}\right|\right)
$$

is the maximum absolute-valued eigenvalue of the Jacobian matrix $A, \beta \approx 1$ is a scalar constant governing the amount of implicit dissipation produced by the matix reconstructions and $I$ is the identity matrix. This splitting is chosen to. insure a nonsingular diagonal inversion and is similar to the Jameson and Turke ${ }^{5}$ splitting that produces diagonally dominant implicit factors for each matrix inversion in every mesh cell ${ }^{4}$. The implicit system of equations is solved in the following two steps:

1) Lower Sweep

$$
\begin{gathered}
{\left[I+\mu \Delta t\left(\delta_{\xi}^{-} A_{1}+\delta_{\eta}^{-} B_{1}+\delta_{\zeta}^{-} C_{1}\right)\right] \Delta \vec{Y}_{i j k}^{n}=} \\
-\Delta t\left(\delta_{\xi} \vec{F}+\bar{\delta}_{\eta} \vec{G}+\bar{\delta}_{s} \vec{H}+\vec{T}\right)_{i j k}^{n}
\end{gathered}
$$

The right hand side of this system, the Residual, is evaluated using the finite volume formulation of the numerical approximation and then inverted by an effectively explicit sweep through the domain in the positive $\xi-, \eta-, \zeta-$ directions. The resulting intermediate variables $\Delta \vec{Y}^{n}$ are then used to start the upper sweep.

\section{2) Upper Sweep}

$$
\left[I+\mu \Delta t\left(\delta_{\xi}^{+} A_{2}+\delta_{\eta}^{+} B_{2}+\delta_{\varsigma}^{+} C_{2}\right)\right] \Delta \vec{W}_{i j k}^{n}=\Delta \vec{Y}_{i j k}^{n}
$$

An effectively explicit sweep through the domain is also required to solve this system, but in directions opposite to those taken in the lower sweep. The resulting flow field corrections are then used to update the flow field.

$$
\vec{W}_{i j k}^{n+1}=\Delta \vec{W}_{i j k}^{n}+\vec{W}_{i j k}^{n}
$$

The LU factorization requires the solution of two block triangular operators each of which, through back substitution, can be reduced to simple $5 \times 5$ matrix systems at every mesh cell. These reduced systems can be written:

\section{1) Lower Sweep}

$$
\begin{gathered}
{\left[I+\mu \Delta t\left(A_{1}+B_{1}+C_{1}\right)\right] \Delta \vec{Y}_{i j k}^{n}=} \\
-\Delta t\left(\bar{\delta}_{\xi} \vec{F}+\bar{\delta}_{\eta} \vec{G}+\bar{\delta}_{\zeta} \vec{H}+\vec{T}\right)_{i j k}^{n} \\
+\mu \Delta t\left(A_{1} \Delta \vec{Y}_{i-1, j, k}^{n}+B_{1} \Delta \vec{Y}_{i, j-1, k}^{n}+C_{1} \Delta \vec{Y}_{i, j, k-1}^{n}\right)
\end{gathered}
$$

\section{2) Upper Sweep}

$$
\begin{gathered}
{\left[I-\mu \Delta t\left(A_{2}+B_{2}+C_{2}\right)\right] \Delta \vec{W}_{i j k}^{n}=\Delta \vec{Y}_{i j k}^{n}} \\
-\mu \Delta t\left(A_{2} \Delta \vec{W}_{i+1, j, k}^{n}+B_{2} \Delta \vec{W}_{i, j+1, k}^{n}+C_{2} \Delta \vec{W}_{i, j, k+1}^{n}\right)
\end{gathered}
$$

and are typically inverted by Gaussian elimination.

\section{Diagonal Inversion}

The solution of the LU factorization can be made more efficient by diagonally inverting its $5 \times 5$ matrix systems. The diagonal inversion is based on the diagonalizing procedure used to classify the Euler equations formally as a hyperbolic system of partial differential equations.

The Euler equations are classified as an hyperbolic system of partial differential equations since a similarity transformation exists such that:

$$
Q^{-1}\left(k_{1} A+k_{2} B+k_{3} C\right) Q=\Lambda
$$

where $\Lambda$ is a diagonal matrix whose elements are real, and $k_{1}, k_{2}$, and $k_{3}$ are scalar constants. For the specific case of $k_{1}=k_{2}=k_{3}=1$, a corresponding diagonalizing transformation can be derived from the general similarity transformation developed for the Euler equations in Cartesian coordinates by Warming, Beam, and Hyett ${ }^{9}$. The matrix $Q$ has elements

$$
\begin{aligned}
& Q_{11}=\hat{l}_{1} \quad Q_{21}=u \hat{l}_{1} \\
& Q_{12}=\hat{l}_{2} \quad Q_{22}=u \hat{l}_{2}-\rho \hat{l}_{3} \\
& Q_{13}=\hat{l}_{3} \\
& Q_{14}=\frac{\rho}{\sqrt{2} c} \quad Q_{24}=\frac{\rho u}{\sqrt{2 c}}+\frac{\rho \hat{l}_{1}}{\sqrt{2}} \\
& Q_{15}=\frac{\rho}{\sqrt{2} c} \quad Q_{25}=\frac{\rho u}{\sqrt{2} c}-\frac{\rho \hat{l}_{1}}{\sqrt{2}} \\
& Q_{31}=v \hat{l}_{1}+\rho \hat{l}_{3} \quad Q_{41}=w \hat{l}_{1}-\rho \hat{l}_{2} \\
& Q_{32}=v \hat{l}_{2} \quad Q_{42}=w \hat{l}_{2}+\hat{l}_{1} \\
& Q_{33}=v \hat{l}_{3}-\rho \hat{l}_{1} \quad Q_{43}=w \hat{l}_{3} \\
& Q_{34}=\frac{\rho v}{\sqrt{2} c}+\frac{\rho \hat{l}_{2}}{\sqrt{2}} \quad Q_{44}=\frac{\rho w}{\sqrt{2} c}+\frac{\rho \hat{l}_{3}}{\sqrt{2}} \\
& Q_{35}=\frac{\rho v}{\sqrt{2} c}-\frac{\rho \hat{l}_{2}}{\sqrt{2}} \quad Q_{45}=\frac{\rho w}{\sqrt{2} c}-\frac{\rho \hat{l}_{3}}{\sqrt{2}} \\
& Q_{51}=\frac{q^{2}}{2} \hat{l}_{1}+\rho v \hat{l}_{3}-\rho w \hat{l}_{2} \\
& Q_{52}=\frac{q^{2}}{2} \hat{l}_{2}-\rho u \hat{l}_{3}+\rho w \hat{l}_{1} \\
& Q_{53}=\frac{q^{2}}{2} \hat{l}_{3}+\rho u \hat{l}_{2}-\rho v \hat{l}_{1} \\
& Q_{54}=\frac{\rho q^{2}}{2 \sqrt{2} c}+\frac{\rho u \hat{l}_{1}}{\sqrt{2}}+\frac{\rho v \hat{l}_{2}}{\sqrt{2}}+\frac{\rho w \hat{l}_{3}}{\sqrt{2}}+\frac{\rho c}{\sqrt{2}(\gamma-1)} \\
& Q_{56}=\frac{\rho q^{2}}{2 \sqrt{2} c}-\frac{\rho u \hat{l}_{1}}{\sqrt{2}}-\frac{\rho v \hat{l}_{2}}{\sqrt{2}}-\frac{\rho w \hat{l}_{3}}{\sqrt{2}}+\frac{\rho c}{\sqrt{2}(\gamma-1)}
\end{aligned}
$$

where

$$
\begin{aligned}
& q^{2}=u^{2}+v^{2}+w^{2} \\
& l_{1}=\xi_{x}+\eta_{x}+\zeta_{x} \\
& l_{2}=\xi_{y}+\eta_{y}+\zeta_{y} \\
& l_{3}=\xi_{x}+\eta_{x}+\zeta_{z}
\end{aligned}
$$




$$
\begin{gathered}
\hat{l}_{1}=\frac{l_{1}}{\sqrt{l_{1}^{2}+l_{2}^{2}+l_{3}^{2}}} \quad \hat{l}_{2}=\frac{l_{2}}{\sqrt{l_{1}^{2}+l_{2}^{2}+l_{3}^{2}}} \\
\hat{l}_{3}=\frac{l_{3}}{\sqrt{l_{1}^{2}+l_{2}^{2}+l_{3}^{2}}}
\end{gathered}
$$

and $c$ is the local speed of sound.

This diagonalizing transformation can be used to produce the diagonal matrix

$$
Q^{-1}(A+B+C) Q=\Lambda
$$

which has elements

$$
\begin{gathered}
\lambda_{11}=\lambda_{22}=\lambda_{33}=U+V+W \\
\lambda_{44}=U+V+W-c \sqrt{l_{1}^{2}+l_{2}^{2}+l_{3}^{2}} \\
\lambda_{55}=U+V+W+c \sqrt{l_{1}^{2}+l_{2}^{2}+l_{3}^{2}} \\
\lambda_{i j}=0 \quad \text { when } \quad i \neq j
\end{gathered}
$$

Applying this local similarity transformation to the lower and upper sweeps produces the following scalar systems:

1) Lower Sweep

$$
\begin{gathered}
{\left[I+\frac{\mu \Delta t}{2}(\Lambda+\beta(|A|+|B|+|C|) I)\right] Q^{-1} \Delta \vec{Y}_{i j k}^{n}=} \\
-\Delta t Q^{-1}\left(\left(\bar{\delta}_{\xi} \vec{F}+\bar{\delta}_{\eta} \vec{G}+\bar{\delta}_{s} \vec{H}+\vec{T}\right)_{i j k}^{n}\right. \\
\left.-\mu\left(A_{1} \Delta \vec{Y}_{i-1, j, k}^{n}+B_{1} \Delta \vec{Y}_{i, j-1, k}^{n}+C_{1} \Delta \vec{Y}_{i, j, k-1}^{n}\right)\right)
\end{gathered}
$$

\section{2) Upper Sweep}

$$
\begin{gathered}
{\left[I-\frac{\mu \Delta t}{2}(\Lambda-\beta(|A|+|B|+|C|) I)\right] Q^{-1} \Delta \vec{W}_{i j k}^{n}=} \\
Q^{-1}\left(\Delta \vec{Y}_{i j k}^{n}-\mu \Delta t\left(A_{2} \Delta \vec{W}_{i+1, j, k}^{n}\right.\right. \\
\left.\left.+B_{2} \Delta \vec{W}_{i, j+1, k}^{n}+C_{2} \Delta \vec{W}_{i, j, k+1}^{n}\right)\right)
\end{gathered}
$$

The lower and upper sweeps have now been transformed into uncoupled systems of scalar equations that retain the stability and time accuracy of the original LU factorization. Unlike in the Diagonalized ADI method, time conservation is not altered by the decoupling process since the similarity transformation is not factored out of the implicit spatial differences. For a time step defined as

$$
\Delta t=\frac{C n}{(|A|+|B|+|C|)}
$$

where $C n$ is the Courant number used in the time marching, the vector components $(m=1, \ldots, 5)$ of the lower and upper sweeps can now be written and solved as follows.

1) Lower $S$ weep

$$
\left(Q^{-1} \Delta \vec{Y}_{i j k}^{n}\right)_{m}=
$$

$$
\frac{\left.\left(\begin{array}{c}
-\Delta t Q^{-1}\left(\left(\vec{\delta}_{\xi} \vec{F}+\vec{\delta}_{\eta} \vec{G}+\bar{\delta}_{\zeta} \vec{H}+\vec{T}\right)_{i j k}^{n}\right. \\
-\mu\left(A_{1} \Delta \vec{Y}_{i-1, j, k}^{n}+B_{1} \Delta \vec{Y}_{i, j-1, k}^{n}+C_{1} \Delta \vec{Y}_{i, j, k-1}^{n}\right)
\end{array}\right)\right)_{m}}{\left(\left(1+\frac{\mu \beta C n}{2}\right) I+\frac{\mu \Delta t}{2} \Lambda\right)_{m}}
$$

2) Upper Sweep

$$
\begin{gathered}
\left(Q^{-1} \Delta \vec{W}_{i j k}^{n}\right)_{m}= \\
\frac{\left(\begin{array}{c}
Q^{-1}\left(\Delta \vec{Y}_{i j k}^{n}-\mu \Delta t\left(A_{2} \Delta \vec{W}_{i+1, j, k}^{n}\right.\right. \\
\left.\left.+B_{2} \Delta \vec{W}_{i, j+1, k}^{n}+C_{2} \Delta \vec{W}_{i, j, k+1}^{n}\right)\right)
\end{array}\right)_{m}}{\left(\left(1+\frac{\mu \beta C n}{2}\right) I-\frac{\mu \Delta t}{2} \Lambda\right)_{m}}
\end{gathered}
$$

and

$$
\vec{W}_{i j k}^{n+1}=Q\left(Q^{-1} \Delta \vec{W}_{i j k}^{n}\right)+\vec{W}_{i j k}^{n}
$$

The solution of these scalar equations requires significantly less computional effort than the original matrix equations. The diagonally inverted lower sweep requires approximately 855 operation counts per mesh cell as opposed to the over 1453 operation counts per mesh cell required by the original lower sweep (a similar reduction is produced in the upper sweep). On a scalar machine, this reduction in operation counts translates into a 30-35 percent reduction in CPU time, but on a vector machine the code can be restructured to produce an even greater increase in efficiency due to increased vectorization. All outward subroutine calls and short inner loops can be eliminated from the lower and upper sweeps, thereby allowing most of the non-recursive parts of the code to vectorize. This decrease in operation counts, together with the partial vectorization of the previously unvectorizable lower and upper sweeps, significantly increases the computational efficiency of the LU factorization.

\section{Initial and Boundary Conditions}

Initial conditions are needed to start the calculations and are chosen to be a uniform flow field based on an initial guess of the upstream flow.

During the residual calculation, a no-flux condition is enforced at the solid boundaries by setting to zero the contravariant velocity component normal to the boundary. This treatment requires that only pressure needs to be specified along solid boundaries and is obtained from a three-dimensional interpretation of the normal momentum anaylsis developed by Rizzi ${ }^{10}$. For a solid boundary aligned with an $\eta-\zeta$ plane, the normal momentum analysis requires that the following equation be satisfied.

$$
a_{1} \frac{\partial P}{\partial \xi}+a_{2} \frac{\partial P}{\partial \eta}+a_{3} \frac{\partial P}{\partial \zeta}=b_{1}+b_{2}
$$

where

$$
\begin{aligned}
& \dot{a_{1}}=\left(D \xi_{x}\right)^{2}+\left(D \xi_{y}\right)^{2}+\left(D \xi_{z}\right)^{2} \\
& a_{2}=D^{2} \xi_{x} \eta_{x}+D^{2} \xi_{y} \eta_{y}+D^{2} \xi_{z} \eta_{z} \\
& a_{3}=D^{2} \xi_{x} \zeta_{x}+D^{2} \eta_{y} \zeta_{y}+D^{2} \xi_{z} \zeta_{z} \\
& b_{1}=\rho D V\left(u \frac{\partial\left(D \xi_{x}\right)}{\partial \eta}+v \frac{\partial\left(D \xi_{y}\right)}{\partial \eta}+w \frac{\partial\left(D \xi_{z}\right)}{\partial \eta}\right) \\
& b_{2}=\rho D W\left(u \frac{\partial\left(D \xi_{x}\right)}{\partial \zeta}+v \frac{\partial\left(D \xi_{y}\right)}{\partial \zeta}+w \frac{\partial\left(D \xi_{z}\right)}{\partial \zeta}\right)
\end{aligned}
$$


For a steady subsonic inflow boundary, total pressure, total temperature and two flow angles are specified while a one-dimensional Rieman invariant

$$
R=q-\frac{2 c}{\gamma-1}
$$

is extrapolated from the interior flow field (similar to the treatment used by Chima ${ }^{11}$ ).

For steady subsonic outflow, the specified boundary condition is a nonreflective treatment, based on the work of Rudy and Strikwerda ${ }^{12}$, that attempts to minimizes unwanted reflected waves from the outflow boundary. Static pressures are obtained from a radial momentum analysis

$$
\frac{1}{\rho} \frac{\partial P}{\partial r}=\frac{U_{\theta}}{r}
$$

( $r$ is radial distance and $U_{\theta}^{2}$ is tangential velocity) and then coupled with the incoming compatibility relation to produce a nonreflective boundary condition

$$
\frac{\partial P}{\partial t}-\frac{\rho \tilde{C}}{\zeta_{z}} \frac{\partial w}{\partial t}+\alpha\left(P-P_{r e f}\right)=0
$$

where

$$
\tilde{C}=c \sqrt{\zeta_{x}^{2}+\zeta_{y}^{2}+\zeta_{z}^{2}}
$$

$P_{\text {ref }}$ is the static pressure obtained from the radial moment um equation and $\alpha \approx 1$ is a scalar constant.

\section{Steady State Calculations}

The calculation of steady state solutions is made more efficient by using local time-stepping and the multigrid method.

Local time-stepping is used to optimize the time step throughout the flow field. A locally varing time step size, based on a constant Courant number, is used to create a warped time integration that can accelerate the calculation to a steady state without affecting the steady state solution.

The multigrid method is incorporated into the Diagonally Inverted $L U$ scheme to accelerate the removal of low frequency errors from the flow solution and thus increase the efficiency of the time-marching procedure. Following the work of Jameson ${ }^{13}$ and Jameson and Yoon ${ }^{14}$, the flow solver is used to smooth out high frequency errors resolvable on any current grid level, while the multigrid method is used to eliminate low frequency errors through a sequence of flow calculations on coarser grids. The multigrid sequencing used is a three-level W-cycle that incorporates a double pass on the coarse grids. Coarse grid boundary conditions are identical to those used on the fine grid with the exception of the inflow/outflow conditions which are updated only on the fine grids. Coarse grid residuals are kept smooth by adding only a constant coefficient second difference artificial dissipation term (the nonlinear blended terms are used only on the fine grid). This treatment attempts to limit the amount of high frequency errors reintroduced into the flow field by the upward interpolation of the coarse grid corrections. The flow solver, in this case the Diagonally Inverted LU scheme, is invoked only once on each grid level and only before transfering the flow field to the next coarser grid. The multigrid cycle defined above requires $41 / 32$ work units of computational effort where work units are normalized by a single flow calculation on the finest grid.

\section{Results}

Numerical results are presented to illustrate the $\mathrm{Di}$ agonally Inverted LU scheme's ability to calculate threedimensional turbomachinery flows and the convergence acceleration produced by the multigrid method.

All results were calculated on $\mathrm{H}$-type grids consisting of $64 \times 16 \times 16$ mesh cells in the throughflow, blade-toblade, and radial directions, respectively. The grids were generated using a modified version of the GRAPE code ${ }^{15}$ originally developed by Sorenson ${ }^{16}$. All calculations were performed on a CRAY X-MP, where a calculation consisting of 250 work units required approximately 0.9 million words of memory and 10 minutes of CPU time. This result is in contrast to the original LU scheme for which a comparable calculation required over 42 minutes of CPU time. The diagonal inversion has significantly reduced the computational effort required to solve the $L U$ factorization and has therefore transformed it into a more efficient numerical scheme.

The first test case was a stationary (rot. $=0$ ) cascade passage whose blade shapes were derived from the NACA 0012 airfoil. The three-dimensional grid shown in Figure 1 is based on a 36 blade axial rotor with a 0.825 hubto-tip radius ratio and a 14.3 tip radius-to-blade chord ratio. Flow conditions of 0.66 exit static-to-inflow total pressure ratio (on the hub, P.R. $=0.66$ ) and axial relative inflow (angy $=90$, angz $=0$ ) were used to produce a transonic flow through the turbomachinery passage. This case is used to illustrate the numerical scheme's ability to calculate transonic flows and the convergence acceleration produced by the multigrid method.

Convergence histories for calculations on a single grid and with the multigrid method are shown in Figures 2 and 3 . Both calculations were run at a Courant number of 10 and with local time-stepping. The logarithm of the flow field error and a measure of the developing supersonic region are plotted versus the work units required to produce these results. The flow field error is characterized by the logarithm of the rms rate of change of the continuity equation on the finest grid (scaled by its initial value), while the developing supersonic region (a measure of global convergence) is represented by the number of grid cells (NSUP) found to contain supersonic flow (scaled by its final value). The calculation performed on a single grid (Figure 2) shows a reduction in the average residual of almost 3.5 orders of magnitude after 800 work units. The supersonic pocket has not yet converged to its final value after 800 work units, which suggests that the flow field error should be further reduced (Res 1 is the calculation's starting residual while $\mathrm{Res} 2$ is the final residual). The multigrid method is incorporated into the time-stepping technique to produce a satisfactory reduction in the flow field error without having to resort to a large number of time steps. Figure 3 shows the convergence history of the three-level W-cycle, where a fully developed supersonic region was produced in approximately 150 work units and 4.5 order of magnitude drop in the flow field error was produced within 250 work units. The use of the multigrid method has clearly accelerated the convergence rate of this steady state calculation.

The blade surface and blade-to-blade Mach number distributions at mid-span are shown in Figures 4 and 5 
to illustrate the accuracy and the shock-capturing capabilities of the Diagonally Inverted LU scheme. Near the blades the flow is accelerated to a peak Mach number of 1.28 and then shocked down to subsonic flow through a shock that is captured over three mesh cells.

The second test case is the Annular Cascade designed and extensively tested at NASA Lewis ${ }^{17,18}$. The computational grid shown in Figure 6 is based on the full annular ring of 36 core turbine stator vanes. The geometry is a $38.10 \mathrm{~mm}$ high untwisted blade of constant profile with an axial chord of $38.23 \mathrm{~mm}$. The stator has a tip diameter of $508 \mathrm{~mm}$ and a $0.85 \mathrm{hub}$-to-tip radius ratio.

Test conditions of ambient axial inflow and a 0.65 hub-static to inlet-total pressure ratio produce a flow field with mean radius inlet and exit critical velocity ratios of 0.231 and 0.778 respectively. To match the upstream flow conditions (an inflow Mach number of 0.211), the Euler calculations were run with a $0.66 \mathrm{hub}$-static to inlet-total pressure ratio.

Figure 7 shows the convergence history of the Annular Cascade calculation (with multigrid) where a drop of 5 orders of magnitude in the flow field error was produced within 250 work units.

The resulting flow field is fully subsonic and compared with experimental data at three spanwise positions. Figures 8,9 , and 10 compare the calculated blade surface static pressure distributions at $13.3,50$, and 86.7 percent span with the experimental data produced by Goldman and Seasholtz ${ }^{18}$. The trailing edge flow is not well captured due to the bluntness of the blade's trailing edge but the overall flow field agrees well with the experimental data. Figure 11 shows the blade-to-blade Mach number distribution at the mid-span position where the flow is turned approximately 67 degrees from the axial inflow. Figure 12 shows the critical velocity ratio distribution along the 50 percent radial span plane at the 150 percent axial chord location. The Euler equations cannot capture the viscous wake effects but the overall flow field does seem to be adequately modelled. The computational results compare well with the experimental data and demonstrate the scheme's ability to calculate complex flows accurately.

\section{Concluding Remarks}

A new Diagonally Inverted LU Implicit scheme has been developed for the three-dimensional unsteady Euler equations. The matrix systems that are normally inverted in the LU scheme are treated by local diagonalizing transformations that decouple them, without a loss of time conservation, into systems of scalar equations. This decoupling significantly reduces the computational effort required to solve the LU approximation and therefore transforms it into a more efficient method of numerically solving the unsteady three-dimensional Euler equations. Results illustrate the scheme's ability to calculate accurately three-dimensional flows through turbomachines and the convergence acceleration produced when coupled with the multigrid method.

\section{References}

[1] Chaussee, D.S., and Pulliam, T.H., "Two-Dimensional Inlet Simulation using a Diagonal Implicit Algorithm," AIAA Journal, Vol. 19, No. 2, pp. 153-159, February 1981.

[2] Obayashi, S., and Fujii, K., "Computation of ThreeDimensional Viscous Transonic Flow with the LU
Factored Scheme," AIAA Paper 85-1510, 7th Computational Fluid Dynamics Conference, Cincinnati, Ohio, July 1985.

(3) Yoon, S., and Jameson, A., "An LU-SSOR Scheme for the Euler and Navier-Stokes Equations,"AIAA Paper 87-0600, 25th Aerospace Sciences Meeting, Reno, Nevada, January 1987.

[4] Yokota, J.W., and Caughey, D.A., "An L-U Implicit Multigrid Algorithm for the Three-Dimensional Euler Equations," AIAA Paper 87-0453, 25th Aerospace Sciences Meeting, Reno, Nevada, January 1987.

[5] Jameson, A., and Turkel, E., "Implicit Schemes and LU Decompositions," Mathematics of Computation, Vol. 37, No. 156, pp. 385-397, October 1981.

(6) Buratynski, E.K., and Caughey, D.A., "An Implicit LU Scheme for the Euler Equations applied to Arbitrary Cascades," AIAA Journal, Vol. 24, No.1, pp.3946, January 1986.

[7] Jameson, A., "Transonic Flow Calculations for Aircraft," in Numerical Methods in Fluid Dynamics Lecture Notes in Mathematics, Vol. 1127, Brezzi,F., ed., pp. 156-242, Springer-Verlag, New York, 1985.

[8] Pulliam, T.H., "Artificial Dissipation Models for the Euler Equations," AIAA Paper 85-0438, 23rd Aerospace Sciences Meeting, Reno, Nevada, January 1985.

[9] Warming, R.F., Beam, R.M., and Hyett, B.J., "Diagonalization and Simultaneous Symmetrization of the Gas-Dynamic Matrices," Mathematics of Computation, Vol. 29, pp. 1037-1045, October 1975.

[10] Rizzi, A., "Numerical Implementation of the SolidBody Boundary Conditions for the Euler Equations," Zeitschrift fuer Angewandte Mathematik und Mechanik, Vol. 58, No. 7, pp. 301-304, July 1978.

(11) Chima, R.V., "Analysis of Inviscid and Viscous Flows in Cascades with an Explicit Multiple-Grid Algorithm," NASA TM 83636, 1984.

[12] Rudy, D.H., and Strikwerda, J.C., "A Nonreflecting Outflow Boundary Condition for Subsonic Navier-Stokes Calculations," Journal of Computational Physics, Vol. 36, No.1, pp. 55-70, June 1980.

(13) Jameson, A., "Solution of the Euler Equations for Tivo-Dimensional Transonic Flow by a Multigrid Method," MAE Report 1613,Princeton University, 1983.

[14] Jameson, A., and Yoon, S., "Multigrid Solution of the Euler Equations Using Implicit Schemes," AIAA Paper 85-0293, Reno, Nevada, January 1985.

[15] Smith, W.A., "Modified Grape Code," Sibley School of Mechanical and Aerospace Engineering FDA Report 87-10, Cornell University, June 1987.

[16] Sorenson, R., "A Computer Program to Generate Two Dimensional Grids About Airfoils and Other Shapes by the use of the Poisson's Equation," NASA TM 81198, 1980.

[17] Goldman, L.J., and McLallin, K.L., "Cold-Air Annular Cascade Investigation of Aerodynamic Performance of Core-Engine-Cooled Turbine Vanes. I: SolidVane Performance and Facility Description," NASA TM X-3224, 1975.

[18] Goldman, L.J., and Seasholtz, R.G., "Laser Anemometer Measurements in a Annular Cascade of Core Turbine Vanes and Comparison with Theory," NASA TP-2018, 1982. 


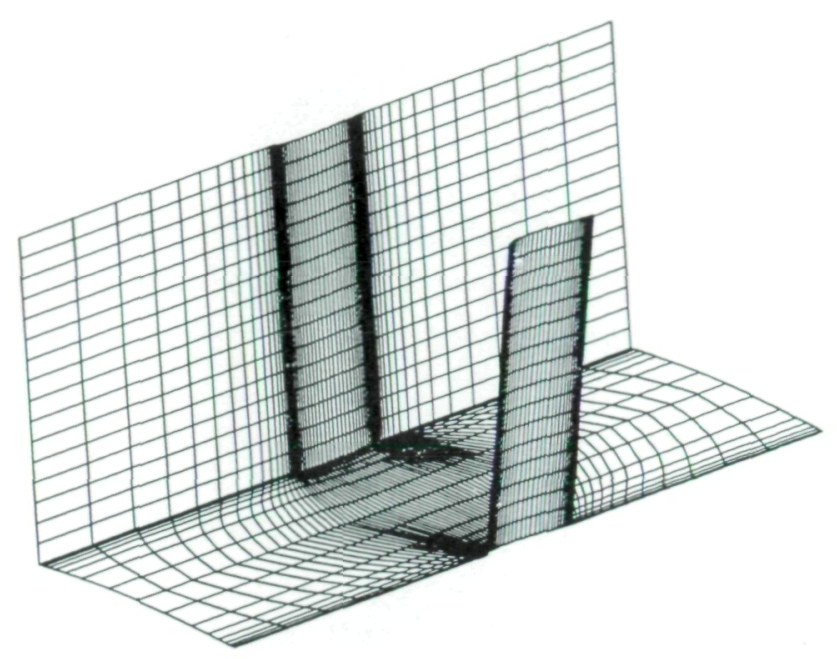

Figure - 1. Computational grid for the NACA 0012 Cascade.
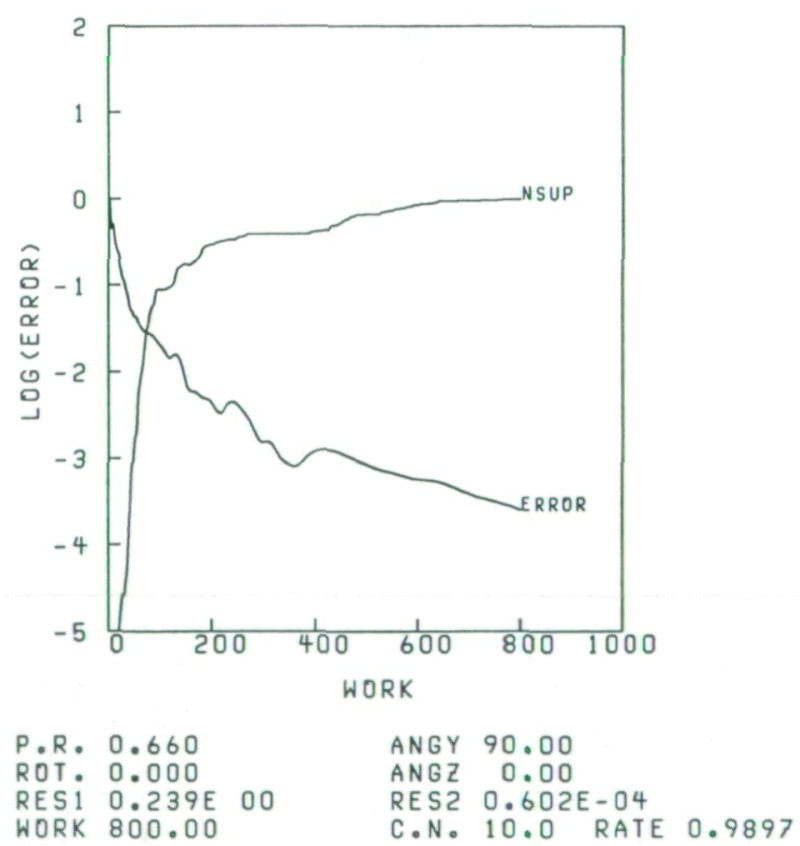

Figure - 2. Convergence history for the single grid calculation.
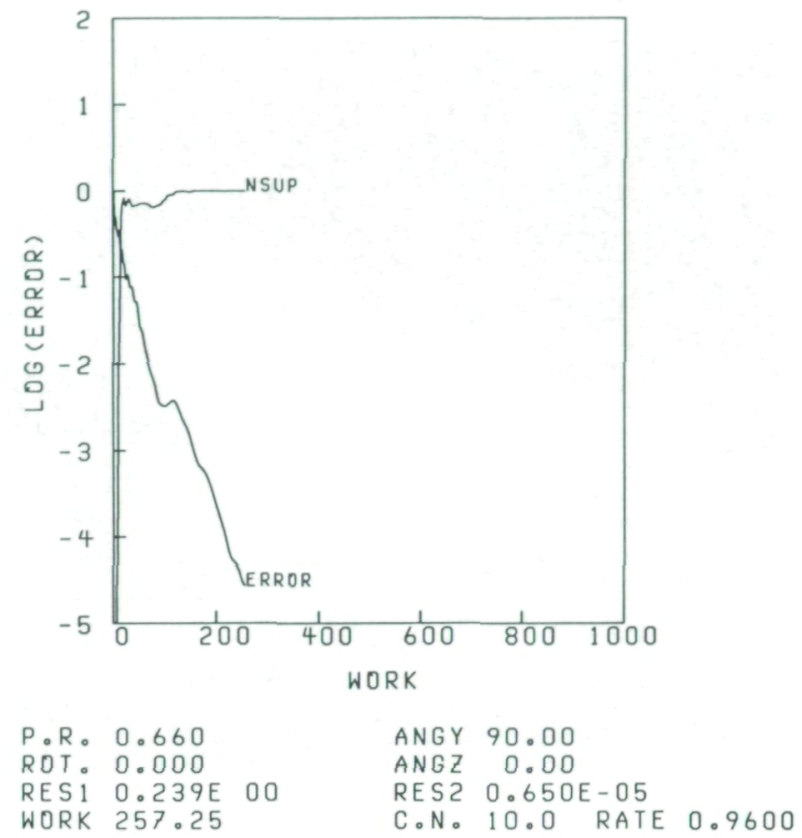

Figure - 3. Convergence history for the multigrid calculation.

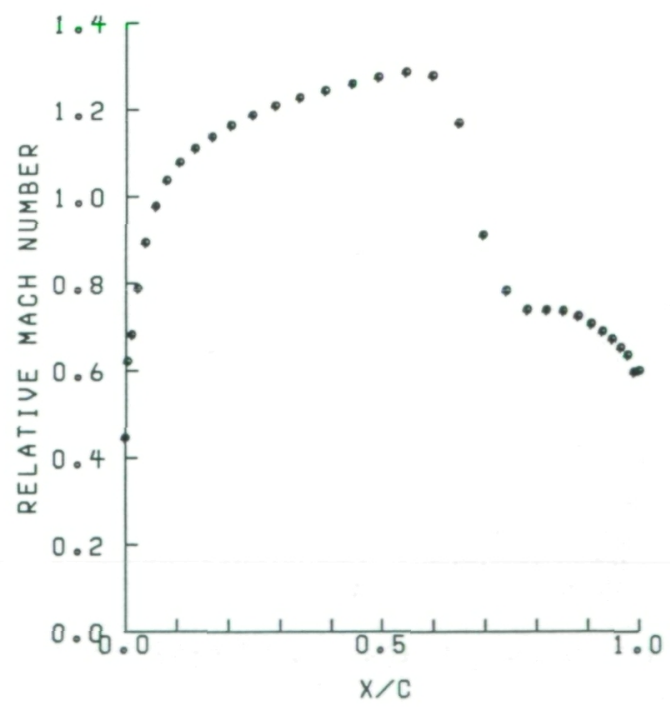

Figure - 4. Mach number blade distribution at 50 percent span. 


\section{OMGENAL PAGE \\ COLOR PHOFOGRAPH}

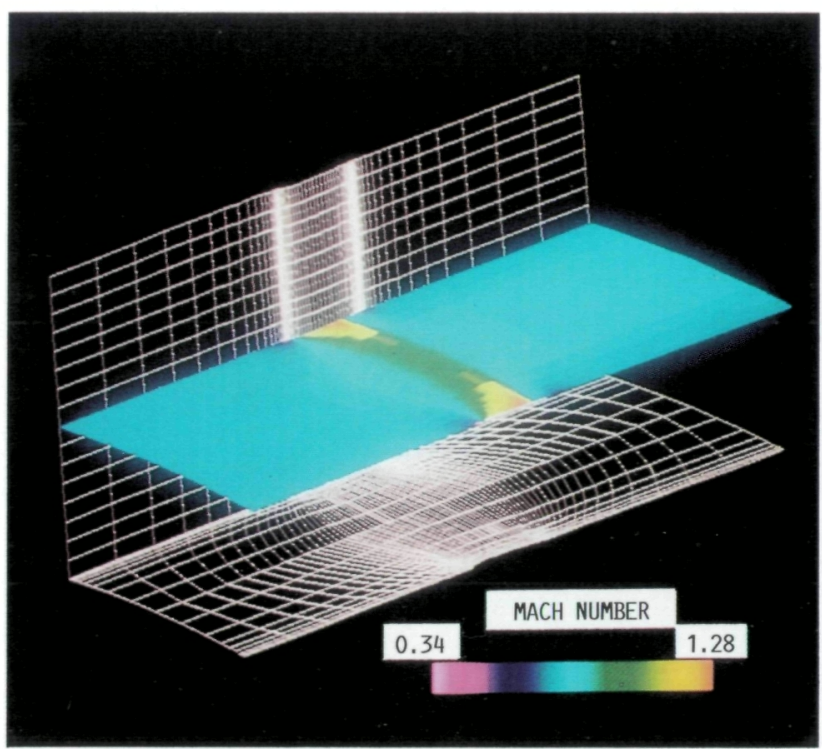

Figure - 5. Mach number distribution at 50 percent span.

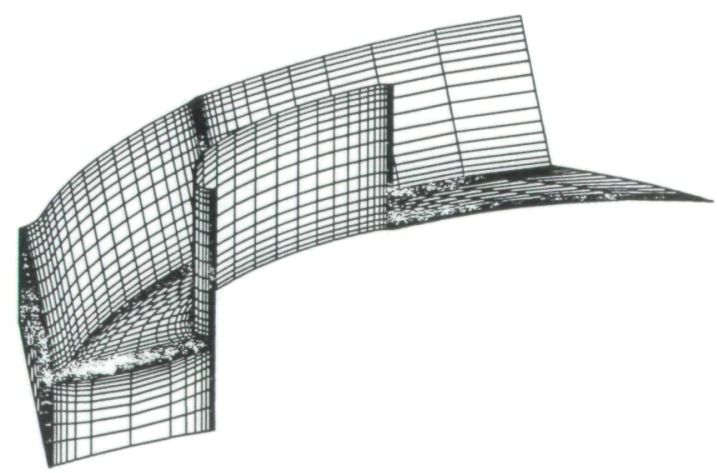

Figure - 6. Computational grid for the Annular Cascade.

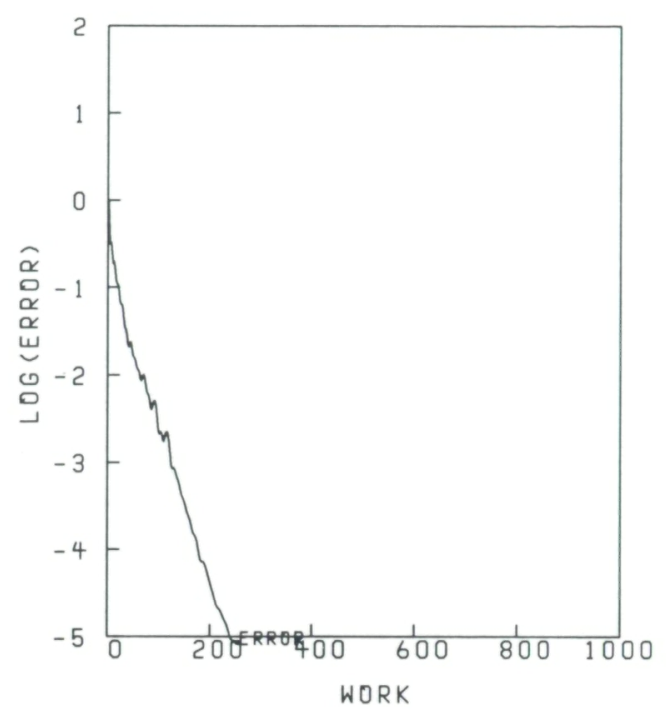

$P . R \cdot 0.660$

ROT \& 0.000

RES1 $0.116 E \quad 01$

WORK 257.25

ANGY 90.00

ANGZ 0.00

RES2 $0,950 E-05$

C.N. 5.0 RATE 0.9555

Figure -7 . Convergence history for the multigrid calculation.

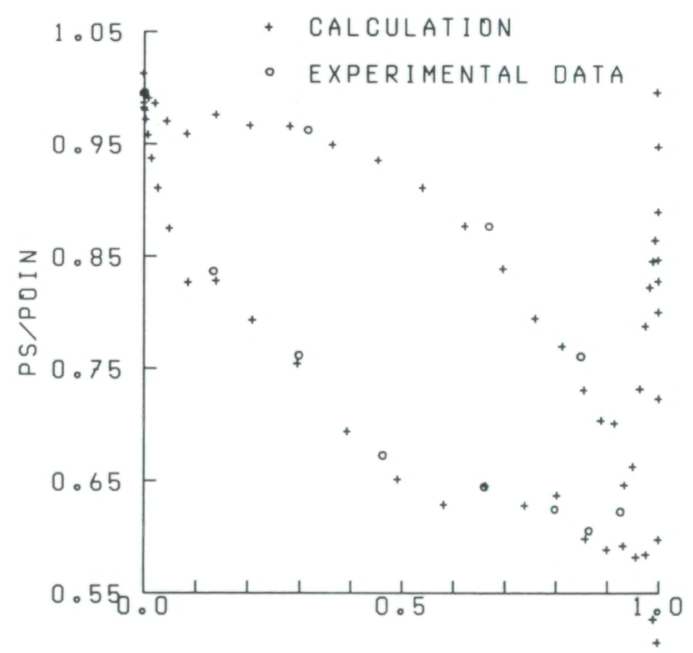

$x / C$

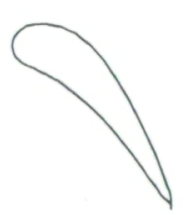

Figure - 8. Static pressure blade distribution at 13.3 percent span. 


\section{ORIGINAL PAGE \\ COLOR PHOFOGRAPH}
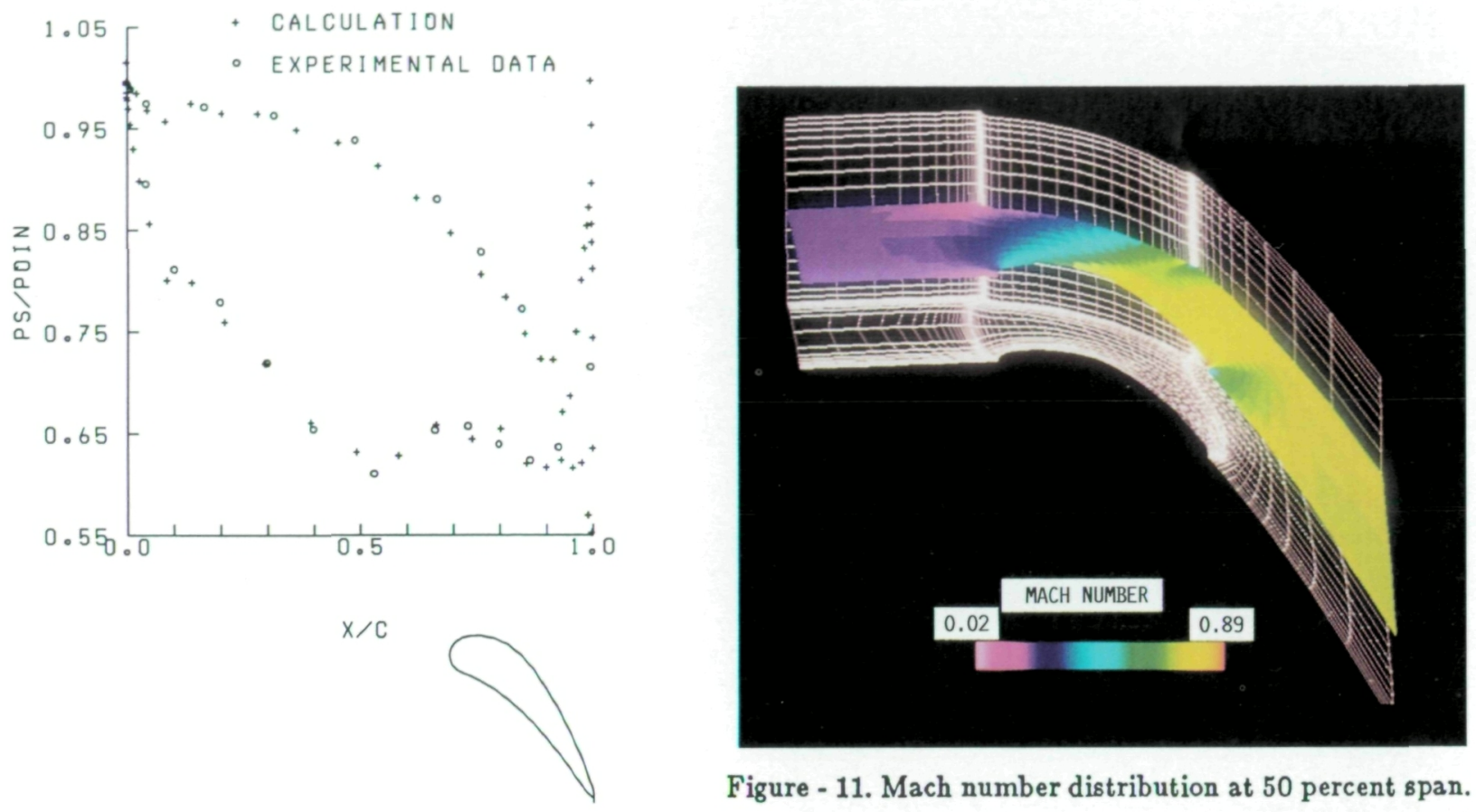

Figure - 9. Static pressure blade distribution at 50 percent span.
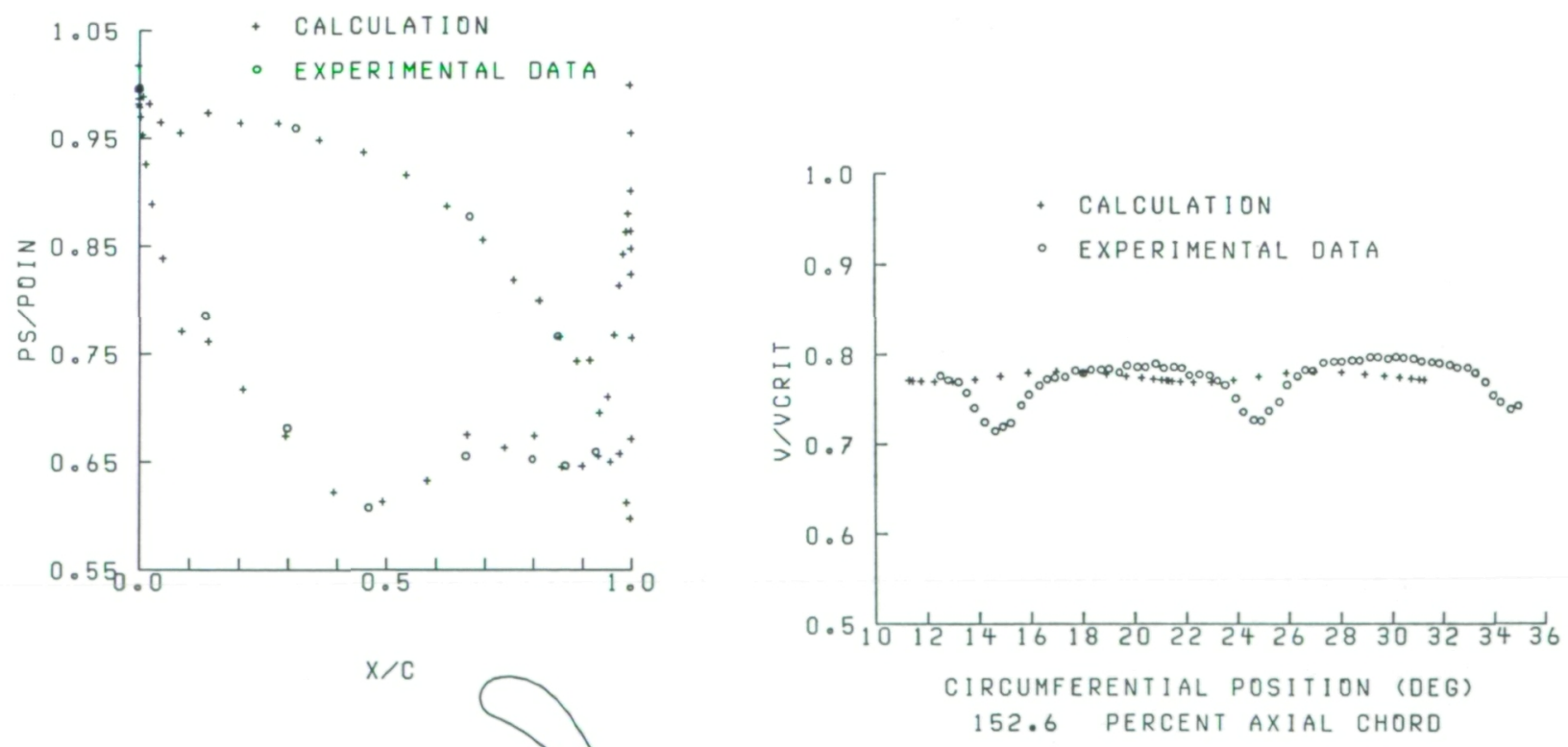

Figure - 12. Critical velocity ratio at 50 percent span.

Figure - 10. Static pressure blade distribution at 86.7 percent span.

Figure - 11. Mach number distribution at 50 percent span. 


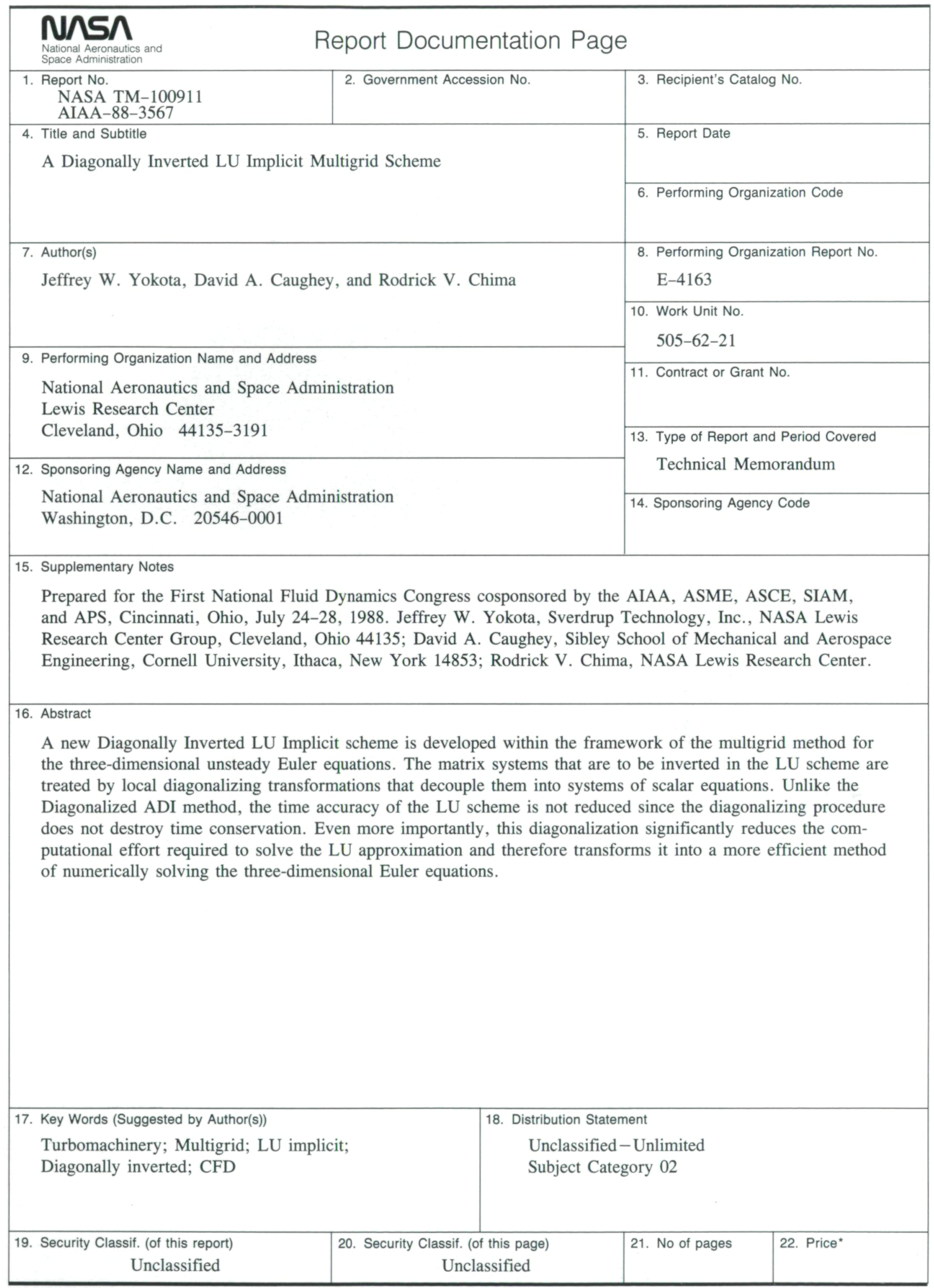




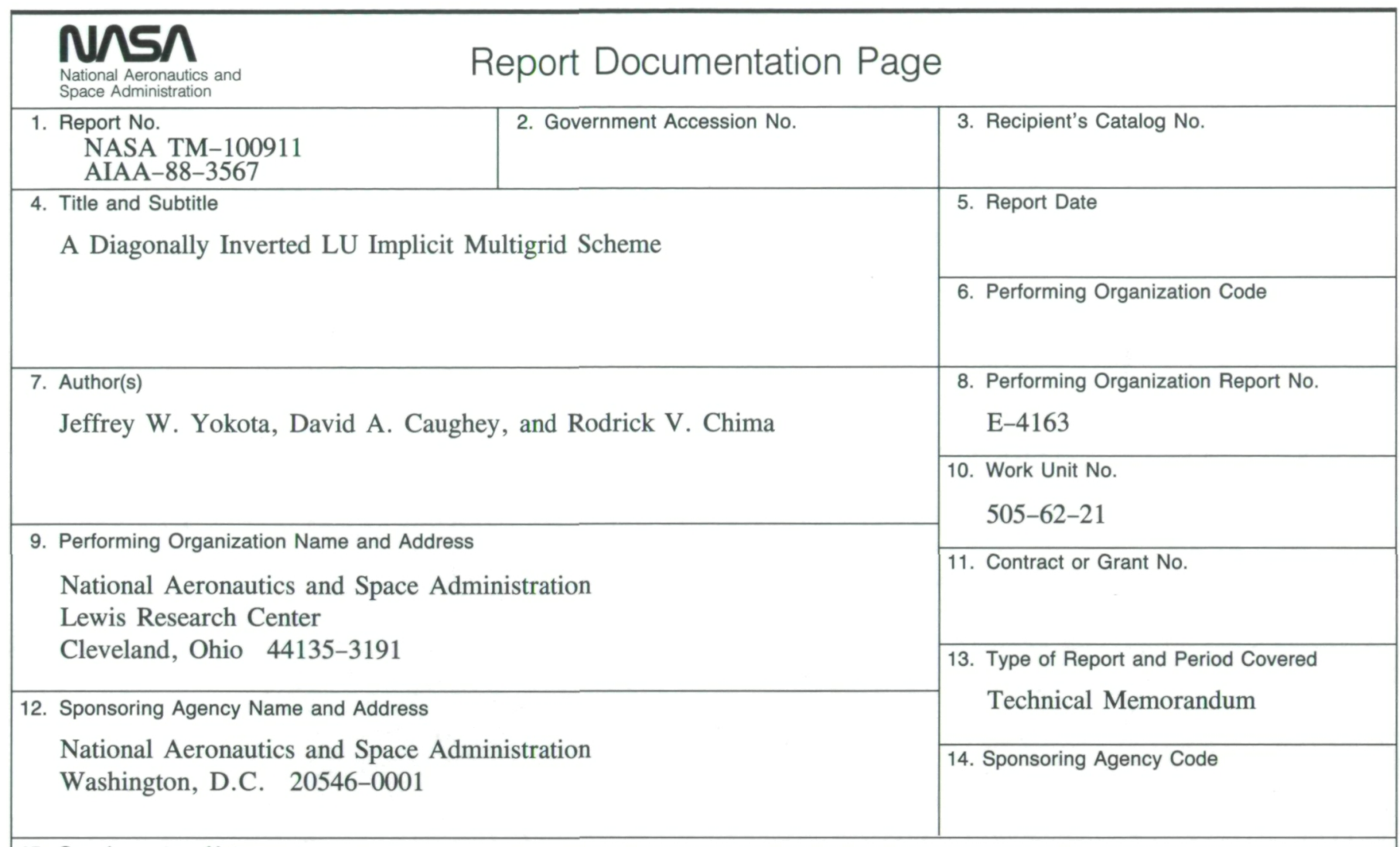

15. Supplementary Notes

Prepared for the First National Fluid Dynamics Congress cosponsored by the AIAA, ASME, ASCE, SIAM, and APS, Cincinnati, Ohio, July 24-28, 1988. Jeffrey W. Yokota, Sverdrup Technology, Inc., NASA Lewis Research Center Group, Cleveland, Ohio 44135; David A. Caughey, Sibley School of Mechanical and Aerospace Engineering, Cornell University, Ithaca, New York 14853; Rodrick V. Chima, NASA Lewis Research Center.

\section{Abstract}

A new Diagonally Inverted LU Implicit scheme is developed within the framework of the multigrid method for the three-dimensional unsteady Euler equations. The matrix systems that are to be inverted in the LU scheme are treated by local diagonalizing transformations that decouple them into systems of scalar equations. Unlike the Diagonalized ADI method, the time accuracy of the LU scheme is not reduced since the diagonalizing procedure does not destroy time conservation. Even more importantly, this diagonalization significantly reduces the computational effort required to solve the LU approximation and therefore transforms it into a more efficient method of numerically solving the three-dimensional Euler equations.

17. Key Words (Suggested by Author(s))

Turbomachinery; Multigrid; LU implicit; Diagonally inverted; CFD
18. Distribution Statement

Unclassified-Unlimited

Subject Category 02 
PRINCETON UNIVERSITY

Department of Mechanical and Aerospace Engineering

Princeton, New Jersey

PR-150-6549-1

PROGRESS REPORT

ON

INTELLIGENT GUIDANCE AND CONTROL FOR

WIND SHEAR ENCOUNTER

Six-Month Progress Report for

NASA Grant No.NAG-1-834

Princeton Account No. 150-6549

July 21,1988

Prepared for:

LANGLEY RESEARCH CENTER

NATIONAL AERONAUTICS AND SPACE ADMINISTRATION

Hampton, Virginia

Submitted by:

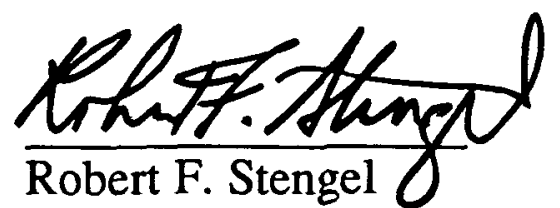

Professor

Principal Investigator 


\section{Summary}

A study of intelligent guidance and control concepts for protecting against the adverse effects of wind shear during aircraft takeoffs and landings has been begun. The principal objectives are to develop methods for assessing the likelihood of wind shear encounter, for deciding what flight path to pursue, and for using the aircraft's full potential for combating wind shear. This study requires the definition of both deterministic and statistical techniques for fusing internal and external information, for making "go/no-go" decisions, and for generating commands to the aircraft's cockpit displays and autopilot for both manually controlled and automatic flight.

The program has begun with the development of a real-time expert system for pilot aiding that is based on the results of the FAA Windshear Training Aids Program. A two-volume manual that presents an overview, pilot guide, training program, and substantiating data provides guidelines for this initial development. The Expert $\underline{\text { System to }} \underline{\text { vvoid }} \underline{\text { Wind }}$ Shear (ESAWS) currently contains over 140 rules and is coded in the LISP programming language for implementation on a Symbolics 3670 LISP Machine.

This six-month progress report includes a brief introduction to ESAWS, as well as numerous appendices that describe the logic and code of the program in considerable detail. This development is especially fluid, and these materials are presented as evidence of progress made to date rather than as documentation of a working system; consequently, the logic is subject to further change, and no guarantee is made that the submitted coding is "bug-free" or even representative of ESAWS's final structure. Program logic is being developed by Professor Stengel and graduate student Alexander Stratton; Mr. Stratton has been responsible for all program coding and has authored a considerable portion of this progress report. 


\section{Table of Contents}

Summary

1. LISP Implementation of Wind Shear Expert System

2. Search Procedures for Wind Shear Expert System

3. Plans for Improvement of Wind Shear Expert System

Appendix A. Parameters for Wind Shear Expert System A-1

Appendix B. Rule Bases for Wind Shear Expert System

Appendix C. LISP Code for Rule Base Executive Functions $\quad c-1$

Appendix D. LISP Code for Rules of Rule-Based Controller D-1

Appendix E. LISP Code for Rules of Wind Shear Risk Assessment $\quad$ E-1

Appendix F. LISP Code for Rule Base Control Functions F-I

Appendix G. Typical Screen Displays from Wind Shear Expert System G-1

Appendix H. Charts for Program Status Report, June, $1988 \quad$ H-1 


\section{LISP Implementation of Wind Shear Expert System}

Wind Shear Expert System software has been written in Common LISP for a Symbolics 3670 LISP machine, using elementary list operations. Execution of the system and input/output operations are carried out in the standard LISP environment, using a "LISP Listener". Currently, the user acts as both flight crew and simulation environment; additions planned for the system, including a FORTRAN aircraft simulation, will add realism for system development and demonstration and will complete the model of flight crew decision-making and control presented in the FAA Windshear Training Aid.

Software for the Wind Shear Expert System consists of parameter definitions (Appendix A), rule bases (Appendix B), and functional LISP procedures (Appendices $\mathrm{C}$ to $\mathrm{F}$ ). Rule bases are defined as LISP variables using the function "defivar". A rule base is simply a list of related rules (see Appendix B for English translations of rules). In the code, each rule is a list containing seven elements. The first element of a rule is the rule's name. Two other elements, called the"if-part" or premise and the "then-part" or consequent, are executable LISP statements that are evaluated if the rule is tried. Two of the elements are character strings that give an English translation of the rule; these strings are typed to a LISP Listener if the premise is evaluated and does not return "NIL".

The two remaining elements of a rule are lists of parameters. One list consists of the variables whose values are set if the consequent is evaluated. This list is a "flag" for a goal-directed search procedure. The other parameter list consists of variables that must have values for the premise to be evaluated; this list is a "flag" for a forward-chaining procedure and is not presently used. Editing features of the Symbolics machine enable nested lists such as the rule bases to be easily entered into files in a neat format that simplifies identification and later modification. Grouping the rules into separate rule bases breaks down the expert system functions into subfunctions in a natural manner.

Expert system parameters store data for the expert system, and are objects of search procedures (see Appendix A). Parameters have been defined using "defvar", and are bound using "setq" in rules and functional procedures. Some parameters are additionally given property values, to expand them to vectors. Some parameters have been grouped to aid variable binding. With hindsight, this has worked well for internal parameters that are given values during search, but it is cumbersome for representing other parameters external to the system. Redefinition of external data, perhaps using "defflavor" and "make-instance", may have advantages and is being considered. 
Functional LISP procedures retrieve rule elements, evaluate rules, conduct goal-directed searches, and bind variables. They are defined using "defun" and are called from a LISP Listener or from within the rule bases. The goal-directed search procedure "get-value-of" is currently the only search procedure being used, although other procedures have been written. "Get-value-of" is called with a goal parameter and a rule base as arguments. It searches the rule base for rules that have a "flag" indicating they can set the value of the goal parameter and tries them usingthe procedure "tryrule". "Get-value-of" is called to begin execution of the expert system and is called frequently within the rule base, resulting in backwardchaining. The development of LISP procedures is essentially complete; however, FORTRAN procedures for simulating, estimating, and controlling aircraft and systems must be implemented. The Symbolics LISP machine permits interaction of LISP and FORTRAN procedures, making the demonstration of FAA Windshear Training Aid procedures and other algorithms possible.

The expert system may be called by a LISP Listener or by a FORTRAN program; the former method has been used up until now. The expert system presently sends documentation to a LISP Listener while it is operating. It prompts the user for inputs through a LISP Listener; the user can give additional inputs by temporarily halting execution of the expert system. This interim arrangement has been adequate for development thus far, but it does not adequately demonstrate the interaction of the expert system with its operating environment.

To adequately demonstrate the system and aid further development, a simulation environment must be implemented that includes aircraft, on-board systems, ground-based information sources, and "mother nature". Modelling events outside of the system adds autonomy and realism to the simulation. To better understand the interaction of the expert system with its environment, a multi-window display must be developed that gives information about several aspects of the system and its environment simultaneously. The Symbolics machine has programs that are intended to streamline the development of multi-window displays; our intention is to quickly develop a simple means of presenting a variety of information. 


\section{Search Procedures for Wind Shear Expert System}

The Wind Shear Expert System, in its current state, is principally a model of pilot and crew decision-making and control, as described in the FAA Windshear Training Aid. The four primary functions of the system are:

1. MONITORING -- Observe sensors, receive reports, alerts, warnings

2. ASSESSMENT -- Detect wind shear encounters, determine if there are signs of wind shear, and if it is safe to continue

3. PLANNING -- Determine what actions and precautions are taken

4. ACTION -- $\quad$ In automatic mode, execute standard, recovery, and go-around procedures; in semi-automatic mode, issue commands to flight directors

These functions are performed as side effects of a goal-directed search for parameter values in a set of rules. A rule, in the context of this report, is an IF-THEN statement; a list of the 141 rules implemented on a Symbolics 3670 LISP machine is enclosed (see Appendix B). A search procedure is simply a process of "trying" rules in a selective fashion. "Trying" a rule means that the premise of a rule is examined to determine its truth or falsehood, and the consequent is examined if the premise is true. Often the consequent is a direction to perform some action or procedure. Typically, the value of a parameter is set in the consequent. The search procedure is given a goal parameter, it finds rules that have consequents that set the value of the goal parameter, and it tries them sequentially, stopping when a rule is tried whose premise is true. If another parameter, whose value has not been determined, is encountered in a rule's premise, that parameter becomes the new goal parameter and a new search commences for the value of that parameter. If none of the rules capable of setting a goal parameter have premises that are true, the user is asked to give a value for the parameter in question. This enables the expert system to be tested with an incomplete rule base.

To demonstrate the actions of the search procedure, consider the following example. The expert system is invoked periodically from a simulation by directing the search procedure to get a value for the parameter "RBC-search-complete". Turning to the section "Parameters for Wind Shear Expert System", we find that "RBC-search-complete" is set in the EXECUTIVE rule base. Now turning to Appendix B, we find two rules that can set this parameter: 
"IF the mission phase is not landed, THEN we must complete executive procedures, and the rule-based controller search is not complete.

IF the mission phase is landed, THEN the rule-based controller search is complete."

Trying the first rule, we must consider the truth or falsehood of the expression, "the mission phase is not landed". To know this, we must find out what the mission phase is. Turning to the list of parameters, we find a parameter "mission-phase" that is set in the MISSION PHASE rule base. The rules within the MISSION PHASE rule base are tried next. When the mission phase has been determined, we return to considering the truth or falsehood of the first premise. If, for example, we determined that the mission phase was not "landed", we would then consider the consequent of the first rule, "we must complete executive procedures, and the rule-based controller search is not complete." The first part of the consequent is actually a call to the search procedure (rules were translated into English to facilitate readability; regrettably, this can make it more difficult to follow the search process), to get a value for the parameter "executive-search-complete". Once this search is complete, the parameter "RBC-search-complete" is set to NIL and the top-level search is finished. 


\section{Plans for Improvement of Wind Shear Expert System}

The Wind Shear Expert System has developed primarily from the top level downward, branching out as the number of rules increases. This approach, one of the advantages of the expert system paradigm for control system development, tends to leave open-ended parameters on the lowest level. The material enclosed does not represent a complete rule-based controller and should be viewed as an interim development. Currently, there are a few parameters that do not have any rules or procedures to give them values. Additionally, there are procedures called by the expert system that are not yet implemented as software. Briefly, a list of major items still needing attention includes:

- Simulation environment

- Improved user interface

- Improved relevance information and testing

- Control system architecture

- Target trajectory generation

- Prediction procedures for aircraft and weather

- Rules to interface with new procedures

Addition of the above items should complete an expert system model of the FAA Windshear Training Aid and provide the basis for further development of a rulebased pilot aid for wind shear survival. 


\section{Appendix A \\ Parameters for Wind Shear Expert System}

Below is a list of the current parameters contained in the wind shear expert system. Parameters have been classified according to how they are given values. These classifications are:

INTERNAL (I) -

PRESET (Pr) -
Parameter is internal to the expert system and gets its value from a goal-directed search procedure.

Parameter is set by flight crew, through the pilot interface.

STATE VARIABLE (S) - $\quad$ Parameter is a state estimate, set by a state estimator.

OUTSIDE $(\mathrm{O})$ - $\quad$ Parameter's value is given to the program by an outside interface.

\section{Internal Parameters}

PARAMETER NAME

TYPE VALUES (INTERPRETED)

EXECUTIVE RULEBASE sets:

RBC-search-complete

executive-procedures-complete
I T (we should keep searching) NIL (we should stop searching)

I T (procedures are not complete) 
MISSION PHASE RULEBASE sets:

mission-phase

current-airport

are-we-committed
I PREFLIGHT-DECISION stage

TAKEOFF (pilot committed) CLIMBOUT (off the ground) CRUISE (takeoff completed) PRELANDING-DECISION stage APPROACH (pilot committed) FLARE (below flare height) LANDING (on runway) LANDED (operations completed)

$\operatorname{Pr} / \mathrm{I}$ [any airport name] (nearest airport)

$\operatorname{Pr} / \mathrm{I} T$ (flight crew committed to takeoff/land)

NIL (flight crew not committed)

WINDSHEAR RULEBASE sets:

microburst-encounter

I $\quad T$ (we are having a microburst encounter)

NIL (no microburst encounter)

DETECTION RULEBASE sets:

detection-complete

I T (search of detection rule base completed) 
DEVIATION RULEBASE sets:

aircraft-response

target-airspeed

airspeed-deviation

target-vertical-speed

vertical-speed-deviation

target-pitch-attitude

attitude-deviation

target-glideslope-displacement
I [list] contains one or more of: MICROBURST (significant deviations):

ABOVE-TARGET-AIRSPEED BELOW-TARGET-AIRSPEED ABOVE-TARGET-VERTICALSPEED

BELOW-TARGET-VERTICALSPEED

ABOVE-TARGET-PITCHATTITUDE

BELOW-TARGET-PITCHATTITUDE

ABOVE-TARGETGLIDESLOPE-DEV.

BELOW-TARGETGLIDESLOPE-DEV. ABOVE-TARGET-THROTTLEPOS.

BELOW-TARGET-THROTTLEPOS.

I [numerical] (knots)

I [numerical] (deviation from target, knots)

I [numerical] $(\mathrm{ft} / \mathrm{min})$

I [numerical] (deviation from target, $\mathrm{ft} / \mathrm{min}$ )

I [numerical] (deg)

I [numerical] (deviation from target, deg)

I . [numerical] (dots) 
glideslope-deviation

throttle-deviation
I [numerical] (deviation from target, dots)

I [numerical] (deviation from target, \%)

COMMUNICATION RULEBASE sets:

new-information-received

incident-reported

tower-informed-goa

tower-informed-delay
I T (new information is received) NIL (no new info received)

I $T$ (if there was an incident, it's reported)

I . T (tower informed of intent to goaround)

NIL (tower not yet informed of intent)

I T (tower informed of intent to delay)

NIL (tower not yet informed)

\section{ACTION RULEBASE sets:}

actions-taken

I T (actions have been taken)

STANDARD PROCEDURES RULEBASE sets:

standard-procedures-complete

configuration-set-for-takeoff

configuration-set-for-landing
I T (standard procedures are now complete)

I T (configuration is set for takeoff) NIL (configuration not set for takeoff)

I T (configuration is set for landing) NIL (configuration is not set for landing) 
glideslope-established

Pr/I T (glideslope has been established) NIL (glideslope is not yet established)

RECOVERY PROCEDURES RULEBASE sets:

recovery-procedures-complete I $\quad \mathrm{T}$ (recovery procedures are now complete)

GO-AROUND PROCEDURES RULEBASE sets:

go-around-procedures-complete I T (go-around procedures are complete)

DELAY PROCEDURES RULEBASE sets:

delay-procedures-complete $\quad$ I $\quad$ T (delay procedures are complete)

PLANNING RULEBASE sets:

$\begin{array}{lll}\text { recommended-procedures } & \text { I } \quad T \text { (mission plan is now updated) }\end{array}$

RUNWAY SELECTION RULEBASE sets:

recommended-runway I [runway id] (new runway selection)

SPEED SELECTION RULEBASE sets:

recommended- $\mathrm{Vr}$

I [numerical] (precautionary rotation speed)

recommended-Va

I [numerical] (precautionary approach speed) 
FLAP SELECTION RULEBASE sets:

recommended-flaps

I [numerical] (precautionary flap setting)

ASSESSMENT RULEBASE sets:

recommended-action

I CONTINUE operations as planned CONSIDER-PRECAUTIONS

DELAY-ALTER-ABORT

EXECUTE-RECOVERY

procedures

RISK RULEBASE sets:

risk

I [numerical] (overall risk factor for wind shear - values correspond to: 0 - no probability of wind shear

1 - low probability of wind shear

2 - medium probability of shear

3 or greater - high probability

WEATHER RISK RULEBASE sets:

weather

suspected-runways
I [numerical] (risk of wind shear derived from weather reports,e.g., SIGMETs, same severity scale as "risk")

I [list of runway id's] (runways reported or observed to have conditions suggesting wind shear) 
LOCALIZED WINDS RULEBASE sets:

localized-strong-winds

I [numerical] values correspond to:

0 - no indications of wind shear

1 - indications, off the flight path

2 - indications, convecting onto path

3 - indications of wind shear on path

HEAVY PRECIPITATION RULEBASE sets:

heavy-precipitation

I [numerical] (same scale as localized-strong winds)

RAINSHOWER RULEBASE sets:

rainshower

I [numerical] (same scale as localized-strong winds)

LIGHTNING RULEBASE sets:

lightning

I [numerical] (same scale as localized-strong winds)

VIRGA RULEBASE sets:

virga

I [numerical] (same scale as localized-strong winds)

TURBULENCE RULEBASE sets:

turbulence

I [numerical] (moderate or greater same scale as localized-strongwinds) 


\section{Preset Parameters}

origin-airport
taxi-speed-limit
destination-airport

flare-agl

$\mathrm{Vr}$

Va

critical-agl

aircraft-type

V1

suitable-runways

Vr-field-length-limit
$\operatorname{Pr} \quad$ [any airport name] (origin airport)

Pr [numerical] (taxi speed limit, knots)

Pr [any airport name] (next destination)

Pr [numerical] (flare altitude, $\mathrm{ft}$ )

$\operatorname{Pr}$ [numerical] (standard rotation speed for this configuration, knots)

Pr [numerical] (standard approach speed for this configuration, knots)

Pr [numerical] (height below which ground contact is major concern, $\mathrm{ft}$ )

Pr [any type in PWG] (type of aircraft)

Pr [numerical] (abort groundspeed, knots)

$\operatorname{Pr}$ [list of runways] (suitable runways)

$\operatorname{Pr} \quad$ [numerical] (field length limit rotation speed for this configuration, knots)

\section{State Variables}

agl

$\mathrm{S}$ [numerical] (alt. above ground, $\mathrm{ft}$ ) 


\author{
airspeed \\ vertical-speed \\ pitch-attitude \\ glideslope-displacement \\ groundspeed \\ runway-remaining

\section{Outside Parameters} \\ wind shear-alert \\ traffic \\ pirep*
}

llwas*

forecast*

temp/dewpt-spread
$S \quad$ [numerical] (current airspeed, $\mathrm{ft}$ )

$\mathrm{S} \quad$ [numerical] $(\mathrm{ft} / \mathrm{min})$

$\mathrm{S} \quad$ [numerical] (deg)

$\mathrm{S} \quad$ [numerical] (dots)

S [numerical] (groundspeed, knots)

$S$ [numerical] (current est. of available runway, $\mathrm{ft}$ )

O T (on-board wind shear detection sys. alert)

NIL (no on-board wind shear det. sys. alert)

$\mathrm{O} \quad \mathrm{T}$ (threatening traffic ahead) NIL (no threatening traffic ahead)

O [numerical] (amount of speed lost/gained by some other flight crew, knots)

O [numerical] (amount of speed differential that triggered some llwas alert, knots)

O [list] (forecast of convective weather)

O [numerical] (degrees F) 
ATIS*

ASWW*

SIGMET*

onboard-radar*

tower-report*

TDWR*

wind-profiler*
O TURBULENCE noted in report RAINSHOWERS noted in report LIGHTNING noted in report VIRGA noted in report HEAVY-PRECIPITATION noted LLWS (low-level wind shear noted)

O TURBULENCE noted in report RAINSHOWERS noted in report LIGHTNING noted in report VIRGA noted in report HEAVY-PRECIPITATION noted LLWS (low-level wind shear noted)

O TURBULENCE noted in report RAINSHOWERS noted in report LIGHTNING noted in report VIRGA noted in report HEAVY-PRECIPITATION noted LLWS (low-level wind shear noted)

O TURBULENCE observed HEAVY-PRECIPITATION observed

O TURBULENCE noted by tower RAINSHOWERS noted by tower LIGHTNING noted by tower VIRGA noted by tower HEAVY-PRECIPITATION noted WINDSHEAR noted by tower

O TURBULENCE noted by TDWR HEAVY-PRECIPITATION noted WINDSHEAR noted by TDWR

O TURBULENCE detected by profiler WINDSHEAR detected by profiler 
forward-looking*

observation

LSS*
O TURBULENCE detected

RAINSHOWERS detected

LIGHTNING detected

VIRGA detected

HEAVY-PRECIPITATION

detected

WINDSHEAR detected

O [numerical] (phenomenon seen by flight crew on flight path, values correspond to:

0 - no suspicious phenomenon seen

1 - localized strong winds seen

2 - heavy precipitation seen

3 - rain showers seen

4 - lightning seen

5 - virga seen

O LIGHTNING detected by lightning sensor

* The parameter also has properties indicating airports and runways to which it pertains. 


\section{Appendix B \\ Rule Bases for Wind Shear Expert System}

\section{EXECUTIVE RULEBASE}

IF the mission phase is not landed,

THEN we must complete executive procedures, and the rule-based controller search is not complete.

IF the mission phase is landed,

THEN the rule-based controller search is complete.

IF the mission phase is preflight decision stage or prelanding decision stage, THEN we must determine recommended procedures, and executive procedures are complete.

IF the mission phase is takeoff,

or the mission phase is climbout, or the mission phase is approach, or the mission phase is flare, or the mission phase is landing rollout, THEN we must take control actions, and executive procedures are complete.

IF the mission phase is takeoff complete,

THEN the current airport is now the destination airport, and the pilot is not committed to land, and executive procedures are complete.

\section{MISSION PHASE RULEBASE}

IF the current airport is the origin airport, and our altitude $(\mathrm{ft})$ is 0 feet, and the pilot is not committed to takeoff, THEN the mission phase is preflight decision stage. 
IF the current airport is the origin airport, and our altitude ( $\mathrm{ft}$ ) is 0 feet, and the pilot is committed to takeoff, THEN the mission phase is takeoff.

IF the current airport is the origin airport, and our altitude ( $\mathrm{ft}$ ) is 0 feet, and our airspeed (knots) is greater than the taxi speed limit (knots), and the flight crew has not committed to takeoff,

THEN the flight crew has passively committed to takeoff, and the mission phase is takeoff.

IF the current airport is the origin airport, and our altitude ( $\mathrm{ft}$ ) is greater than 0 feet, and our altitude (ft) is less than 1000 feet,

THEN the mission phase is climbout.

IF the current airport is the origin airport, and our altitude (ft) is greater than 1000 feet,

THEN the mission phase is takeoff complete.

IF the current airport is the destination airport, and our altitude $(\mathrm{ft})$ is greater than $1000 \mathrm{feet}$, and the flight crew is not committed to land,

THEN the mission phase is prelanding decision stage.

IF the current airport is the destination airport, and our altitude ( $\mathrm{ft}$ ) is below 1000 feet., and we are not flight crew is not committed to land,

THEN the flight crew has passively committed to land, and the mission phase is approach.

IF the current airport is the destination airport, and our altitude (ft) is less than 1000 feet, and our altitude $(\mathrm{ft})$ is above flare altitude $(\mathrm{ft})$, and the flight crew is committed to land,

THEN the mission phase is approach.

IF the current airport is the destination airport, and our altitude (ft) is below flare altitude ( $\mathrm{ft}$ ), and the flight crew is committed to land, THEN the mission phase is flare. 
IF the current airport is the destination airport, and our altitude ( $\mathrm{ft}$ ) is 0 feet, and our airspeed (knots) is above the taxi speed limit (knots),

THEN the mission phase is landing roll.

IF the current airport is the destination airport, and our altitude ( $\mathrm{ft}$ ) is 0 feet, and our airspeed (knots) is below the taxi speed limit (knots), THEN the mission phase is landed.

\section{WINDSHEAR RULEBASE}

IF the search of the detection rulebase is complete, and the aircraft trajectory is deviating significantly from the target trajectory,

THEN there is a microburst encounter.

IF a wind shear alert is sounded by an on-board wind shear detection system, THEN there is a microburst encounter.

IF a microburst encounter has already begun, and the aircraft trajectory is deviating somewhat from the target trajectory, THEN there is a microburst encounter.

IF a microburst encounter has not begun, and the aircraft trajectory is not deviating significantly from the target trajectory,

THEN there is no microburst encounter.

\section{DETECTION RULEBASE}

IF the mission phase is approach or prelanding decision stage,

THEN airspeed deviations must be monitored, and vertical speed deviations must be monitored, and pitch attitude deviations must be monitored, and glideslope displacement deviations must be monitored, and throttle position deviations must be monitored, and the search of the detection rulebase is complete. 
IF the mission phase is neither approach nor prelanding decision stage,

THEN airspeed deviations must be monitored, and vertical speed deviations must be monitored, and pitch attitude deviations must be monitored and the search of the detection rulebase is complete.

\section{DEVIATION RULEBASE}

IF airspeed (knots) is below target airspeed (knots) by more than 15 knots, THEN the aircraft is deviating significantly from the target trajectory.

IF airspeed (knots) is above target airspeed (knots) by more than 15 knots; THEN the aircraft is deviating significantly from the target trajectory.

IF airspeed (knots) is below target airspeed (knots) by between 5 and 15 knots, THEN the aircraft is deviating somewhat from the target trajectory, and the type of deviation includes "below target airspeed".

IF airspeed (knots) is above target airspeed (knots) by between 5 and 15 knots, THEN the aircraft is deviating somewhat from the target trajectory, and the type of deviation includes "above target airspeed".

IF vertical speed $(\mathrm{ft} / \mathrm{min})$ is below target vertical speed $(\mathrm{ft} / \mathrm{min})$ by more than 500 $\mathrm{ft} / \mathrm{min}$,

THEN the aircraft is deviating significantly from the target trajectory.

IF vertical speed ( $\mathrm{ft} / \mathrm{min}$ ) is above target vertical speed $(\mathrm{ft} / \mathrm{min})$ by more than 500 ft./min.,

THEN the aircraft is deviating significantly from the target trajectory.

IF vertical speed (ft/min) is below target vertical speed ( $\mathrm{ft} / \mathrm{min}$ ) by between 200 and $500 \mathrm{ft} . / \mathrm{min}$.,

THEN the aircraft is deviating somewhat from the target trajectory, and the type of deviation includes "below target vertical speed".

IF vertical speed (ft/min) is above target vertical speed $(\mathrm{ft} / \mathrm{min})$ by between 200 and $500 \mathrm{ft} . / \mathrm{min}$.,

THEN the aircraft is deviating somewhat from the target trajectory, and the type of deviation includes "above target vertical speed".

IF pitch attitude (deg) is below target pitch attitude (deg) by more than 5 degrees, THEN the aircraft is deviating significantly from the target trajectory. 
IF pitch attitude (deg) is above target pitch attitude (deg) by more than 5 degrees, THEN the aircraft is deviating significantly from the target trajectory.

IF pitch attitude (deg) is below target pitch attitude (deg) by between 2 and 5 degrees,

THEN the aircraft is deviating somewhat from the target trajectory, and the type of deviation includes "below target pitch attitude".

IF pitch attitude (deg) is above target pitch attitude (deg) by between 2 and 5 degrees,

THEN the aircraft is deviating somewhat from the target trajectory, and the type of deviation includes "above target pitch attitude".

IF glideslope displacement (dots) is below target glideslope displacement (dots) by more than 1 dot,

THEN the aircraft is deviating significantly from the target trajectory.

IF glideslope displacement (dots) is above target glideslope displacement (dots) by more than 1 dot,

THEN the aircraft is deviating significantly from the target trajectory.

IF glideslope displacement (dots) is below target glideslope displacement (dots) by between 0.4 and 1 dot,

THEN the aircraft is deviating somewhat from the target trajectory, and the type of deviation includes "below target glideslope displacement".

IF glideslope displacement (dots) is above target glideslope displacement (dots) by between 0.4 and 1 dot,

THEN the aircraft is deviating somewhat from the target trajectory, and the type of deviation includes "below target glideslope displacement".

\section{COMMUNICATION RULEBASE}

IF the outside interface indicates new information, THEN new information has been received.

IF the outside interface does not indicate new information, THEN no new information has been received. 
IF there was a microburst encounter, and there is no longer a microburst encounter, THEN the incident must be recorded now.

IF the above premise is not true,

THEN there is no need to report an incident.

\section{ACTION RULEBASE}

IF we are having a microburst encounter, and guidance mode is automatic,

THEN recovery procedures must be completed, and control actions are taken.

IF we are not having a microburst encounter, and guidance mode is automatic, and recommended procedures have been determined, and standard procedures are recommended,

THEN standard procedures must be completed, and control actions are taken.

IF we are not having a microburst encounter, and guidance mode is automatic, and go-around procedures are recommended,

THEN go-around procedures must be completed, and control actions are taken.

IF we are not having a microburst encounter, and delaying procedures are recommended,

THEN delaying procedures must be completed, and control actions are taken.

IF we are having a microburst encounter, and guidance mode is semi-automatic,

THEN semi-automatic recovery procedures must be completed, and control actions are taken. 
IF we are not having a microburst encounter, and guidance mode is semi-automatic, and recommended procedures have been determined, and standard procedures are recommended,

THEN semi-automatic standard procedures must be completed, and control actions are taken.

IF we are not having a microburst encounter, and guidance mode is semi-automatic, and go-around procedures are recommended,

THEN semi-automatic go-around procedures must be completed, and control actions are taken.

\section{STANDARD PROCEDURES RULEBASE}

IF the mission phase is takeoff, and the configuration has not been set for the takeoff roll,

THEN the configuration must be set for the takeoff roll, and standard procedures are complete.

IF the mission phase is takeoff, and the airspeed (knots) is below $\operatorname{Vr}$ (knots),

THEN takeoff control laws must be executed, and standard procedures are complete.

IF the mission phase is takeoff, and the airspeed (knots) is above $\operatorname{Vr}$ (knots),

THEN rotation control laws must be executed, and standard procedures are complete.

IF the mission phase is climbout,

THEN climbout control laws must be executed, and standard procedures are complete.

IF the mission phase is approach, and the configuration has not been set for landing,

THEN the configuration must be set for landing, and standard procedures are complete. 
IF the mission phase is approach, and the glideslope has not been established,

THEN we must complete procedures to establish the glideslope, and standard procedures are complete.

IF the mission phase is approach, and the glideslope has been established, THEN approach control laws must be executed, and standard procedures are complete.

IF the mission phase is flare, THEN flare control laws must be executed, and standard procedures are complete.

IF the mission phase is landing roll, THEN landing control laws must be executed, and standard procedures are complete.

\section{RECOVERY PROCEDURES RULEBASE}

IF the altitude ( $\mathrm{ft}$ ) is above critical ( $\mathrm{ft}$ ), and the aircraft type is an L-1011, and the mission phase is takeoff,

THEN controls must be configured for a recovery, and the desired thrust setting is maximum rated, and the desired pitch attitude (deg) is 17.5 degrees, and recovery procedures are complete.

IF the altitude is above critical, and the aircraft is not an L-1011, or the aircraft is an L-1011 and the mission phase is approach, THEN controls must be configured for a recovery, and the desired thrust setting is maximum rated, and the desired pitch attitude (deg) is 15 degrees, and recovery procedures are complete. 
IF the altitude is below critical, and the mission phase is not takeoff or landed,

THEN controls must be configured for a recovery, and the desired thrust setting is overboost, and the desired pitch attitude (deg) is 20 degrees, and recovery procedures are complete.

IF the mission phase is takeoff, and the groundspeed (knots) is less than V1 (knots),

THEN controls must be configured for takeoff abort, and the desired thrust setting is reverse, and recovery procedures are complete.

IF the mission phase is takeoff, and the groundspeed (knots) is greater than V1 (knots), and the airspeed (knots) is less than $\operatorname{Vr}$ (knots), and the runway remaining $(\mathrm{ft})$ is greater than 2000 feet,

THEN controls must be configured for takeoff, and the desired thrust setting is maximum rated, and recovery procedures are complete.

IF the mission phase is takeoff, and the airspeed (knots) is greater than $\mathrm{Vr}$ (knots), THEN controls must be configured for a recovery, and the desired thrust setting is maximum rated, and the desired pitch attitude (deg) is 15 degrees, and recovery procedures are complete.

IF the mission phase is takeoff, and the runway remaining ( $\mathrm{ft}$ ) is less than 2000 feet, THEN controls must be configured for a recovery, and the desired thrust setting is maximum rated, and the desired pitch attitude (deg) is 15 degrees, and recovery procedures are complete. 


\section{GO-AROUND PROCEDURES RULEBASE}

IF the tower has not been informed of our intent to go-around, and there is no traffic in our way,

THEN go-around control laws must be executed, and the tower must be informed of our intent to go-around, and go-around procedures are complete.

IF the tower has not been informed of our intent to go-around, and there is traffic in our way, and our altitude is above critical,

THEN approach control laws must be executed, and the tower must be informed of our intent to go-around, and go-around procedures are complete.

IF the tower has not been informed of our intent to go-around, and there is traffic in our way, and our altitude is below critical,

THEN go-around control laws must be executed, and the tower must be informed of our intent to go-around, and go-around procedures are complete.

IF the tower has been informed of our intent to go-around, THEN go-around control laws are in effect, and go-around procedures are complete.

\section{DELAY PROCEDURES RULEBASE}

IF the tower has not been informed of our intent to delay, and our ETA at the airport (min) is less than 20 minutes,

THEN the tower must be informed of our intent to delay, and delay procedures are complete.

IF the tower has been informed of our intent to delay, THEN delay procedures are complete. 


\section{PLANNING RULEBASE}

IF the recommended action is to delay, alter, or abort, and the mission phase is preflight decision stage, or the mission phase is prelanding decision stage,

THEN delaying procedures are recommended.

IF the recommended action is to delay, alter, or abort, and the mission phase is takeoff, or the mission phase is climbout, or the mission phase is flare, or the mission phase is landing roll,

THEN standard procedures are recommended.

IF the recommended action is to delay, alter, or abort, and the mission phase is approach, and the altitude (ft) is above decision height ( $\mathrm{ft}$ ),

THEN go-around procedures are recommended.

IF the recommended action is to delay, alter, or abort, and the mission phase is approach, and the altitude ( $\mathrm{ft}$ ) is below decision height ( $\mathrm{ft}$ ),

THEN standard procedures are recommended.

IF the recommended action is to take precautions, and precautions have already been taken, or the flight crew has rejected precautions,

THEN standard procedures are recommended.

IF the recommended action is to take precautions, and the mission phase is preflight decision stage,

THEN we must determine the best runway, and we must determine the best flap setting (deg), and we must determine the best Vr (knots), and the precautions must be accepted or rejected by the flight crew.

IF the recommended action is to take precautions, and the mission phase is prelanding decision stage, THEN we must stabilize approach at 1000 feet, and we must determine the best flap setting (deg), and we must determine the best $\mathrm{Va}$ (knots), and the precautions must be accepted or rejected by the flight crew. 
IF the recommended action is to take precautions, and the mission phase is not preflight or prelanding decision stage, THEN standard procedures are recommended.

IF the recommended action is to continue, and the mission phase is approach, and altitude $(\mathrm{ft})$ is below critical altitude $(\mathrm{ft})$, and the glideslope has not been established,

THEN go-around procedures are recommended.

IF the recommended action is to continue, and the mission phase is approach, and the altitude ( $\mathrm{ft}$ ) is above the decision altitude ( $\mathrm{ft}$ ), and the weighted norm of the 2-sigma touchdown dispersion ( $\mathrm{ft}$ ) is greater than the dispersion tolerance $(\mathrm{ft})$,

THEN go-around procedures are recommended.

IF the recommended action is to continue, and the above premises are not true,

THEN standard procedures are recommended.

\section{RUNWAY RULEBASE}

IF some of the suitable runways are suspected of wind shear, THEN the best runway is the longest of the suitable runways not suspected.

IF none of the suitable runways are suspected of wind shear, THEN the best runway is the longest of the suitable runways.

\section{SPEED RULEBASE}

IF field-length-limit $\mathrm{Vr}$ (knots) is greater than the standard $\mathrm{Vr}$ (knots), and is less than 20 knots greater than the standard $\mathrm{Vr}$ (knots), THEN the best $\operatorname{Vr}$ (knots) is the field-length-limit $\operatorname{Vr}$ (knots).

IF field-length-limit $\mathrm{Vr}$ (knots) is more than 20 knots greater than the standard $\mathrm{Vr}$ (knots),

THEN the best $\operatorname{Vr}$ (knots) is 20 knots plus the standard $\operatorname{Vr}$ (knots). 
IF this rule is fired,

THEN the best Va (knots) is 20 knots plus the standard Va (knots).

\section{FLAP RULEBASE}

IF the mission phase is preflight decision stage, and the aircraft type is a B727,

THEN the best flap setting (deg) is 15 degrees.

(12 more rules like the above, for different A/C configurations)

\section{ASSESSMENT RULEBASE}

IF there is no new information received, or new information has been received, and all of the rules in the risk rulebase have been tried, and the overall risk factor is 0 (none),

THEN the recommended action is to continue.

IF the overall risk factor is 3 or greater (high),

THEN the recommended action is to delay, alter, or abort.

IF the overall risk factor is 2 (medium),

THEN the recommended action is to consider precautions.

IF the overall risk factor is 1 (low),

THEN the recommended action is to continue.

\section{RISK RULEBASE}

IF this rule is fired,

THEN the overall risk factor is set to 0 and the list of causes is set to nil.

IF there is an indication of convective weather on our flight path, THEN all of the rules in the weather risk rulebase must be tried, and the weather risk factor is added to the overall risk factor, and weather is added to the list of causes. 
IF there is a pilot report of speed loss (knots) less than 15 knots (but nonzero), and this report is relevant to our current flight path,

THEN the overall risk factor is increased by 2 , and the current runway is added to the list of suspected runways, and the pirep is added to the list of causes.

IF there is a pilot report of speed loss (knots) greater than 15 knots, and this report is relevant to our current flight path,

THEN the overall risk factor is increased by 3 , and the current runway is added to the list of suspected runways, and the pirep is added to the list of causes.

IF there is a LLWAS alert with a speed differential (knots) less than 20 knots, and this alert is relevant to our current flight path,

THEN the overall risk factor is increased by 2 , and the current runway is added to the list of suspected runways, and the LLWAS alert is added to the list of causes.

IF there is a LLWAS alert with a speed differential (knots) greater than 20 knots, and this alert is relevant to our current flight path,

THEN the overall risk factor is increased by 3 , and the current runway is added to the list of suspected runways, and the LLWAS alert is added to the list of causes.

IF there is a pilot report of speed loss (knots) less than 15 knots (but nonzero), and this report is near but not on our current flight path,

THEN the overall risk factor is increased by 1 , and the current runway is added to the list of suspected runways, and the pirep is added to the list of causes.

IF there is a pilot report of speed loss (knots) greater than 15 knots, and this report is near but not on our current flight path,

THEN the overall risk factor is increased by 2 , and the current runway is added to the list of suspected runways, and the pirep is added to the list of causes.

IF there is a LLWAS alert with a speed differential (knots) less than 20 knots, and this alert is near but not on our current flight path,

THEN the overall risk factor is increased by 1 , and the current runway is added to the list of suspected runways, and the LLWAS alert is added to the list of causes. 
IF there is a LLWAS alert with a speed differential (knots) greater than 20 knots, and this alert is near but not on our current flight path,

THEN the overall risk factor is increased by 2 , and the current runway is added to the list of suspected runways, and the LLWAS alert is added to the list of causes.

IF there is a forecast of convective weather on our flight path,

THEN the overall risk factor is increased by 1 , and forecast is added to the list of causes.

\section{WEATHER RISK RULEBASE}

IF this rule is fired,

THEN the weather risk factor is reset to zero.

IF there are indications of localized strong winds on our flight path, THEN the weather risk factor is increased by 3 , and the affected runways are added to the list of suspected runways.

IF there are indications of heavy precipitation on our flight path, THEN the weather risk factor is increased by 3 , and the affected runways are added to the list of suspected runways.

IF there are indications of a rain shower on our flight path, THEN the weather risk factor is increased by 2 , and the affected runways are added to the list of suspected runways.

IF there are indications of lightning on our flight path, THEN the weather risk factor is increased by 2 , and the affected runways are added to the list of suspected runways.

IF there are indications of virga on our flight path, THEN the weather risk factor is increased by 2 , and the affected runways are added to the list of suspected runways.

IF there are indications of moderate or greater turbulence on our flight path, THEN the weather risk factor is increased by 2 , and the affected runways are added to the list of suspected rinways. 
IF the ATIS report indicates a temperature/dew point spread $\left({ }^{0} \mathrm{~F}\right)$ greater than $30 \mathrm{OF}$,

THEN the weather risk factor is increased by 2 ,

and the affected runways are added to the list of suspected runways.

IF there are indications of localized strong winds near but not on our flight path, THEN the weather risk factor is increased by 2 , and the affected runways are added to the list of suspected runways.

IF there are indications of heavy precipitation near but not on our flight path, THEN the weather risk factor is increased by 2 , and the affected runways are added to the list of suspected runways.

IF there are indications of rain showers near but not on our flight path, THEN the weather risk factor is increased by 1 , and the affected runways are added to the list of suspected runways.

IF there are indications of lightning near but not on our flight path, THEN the weather risk factor is increased by 1 , and the affected runways are added to the list of suspected runways.

IF there are indications of virga near but not on our flight path, THEN the weather risk factor is increased by 1 , and the affected runways are added to the list of suspected runways.

IF there are indications of moderate or greater turbulence near our flight path, THEN the weather risk factor is increased by 1 , and the affected runways are added to the list of suspected runways.

\section{LOCALIZED WINDS RULEBASE}

IF ATIS information indicates low-level wind shear, and the wind shear is on our flight path, or an Aviation Severe Weather Watch indicates low-level wind shear, and the wind shear is on our flight path,

or a SIGMET indicates low-level wind shear, and the wind shear is on our flight path,

or a tower report indicates low-level wind shear, and the wind shear is on our flight path,

or a Terminal Doppler Weather Radar indicates low-level wind shear, and the wind shear is on our flight path, 
or a wind profiler indicates low-level wind shear, and the wind shear is on our flight path,

or a forward-looking system indicates low-level wind shear, and the wind shear is on our flight path,

or a flight crew observation indicates low-level wind shear on our flight path, THEN there are indications of localized strong winds on our flight path.

IF ATIS information indicates low-level wind shear, and the wind shear is near but not on on our flight path, or an Aviation Severe Weather Watch indicates low-level wind shear, and the wind shear is near but not on our flight path, or a SIGMET indicates low-level wind shear, and the wind shear is near but not on our flight path,

or a tower report indicates low-level wind shear, and the wind shear is near but not on our flight path,

or a Terminal Doppler Weather Radar indicates low-level wind shear, and the wind shear is near but not on our flight path,

or a wind profiler indicates low-level wind shear, and the wind shear is near but not on our flight path, or a forward-looking system indicates low-level wind shear, and the wind shear is near but not on our flight path,

or a flight crew observation indicates low-level wind shear near our flight path, THEN there are indications of localized strong winds near our flight path.

\section{HEAVY PRECIPITATION RULEBASE}

IF ATIS information indicates heavy precipitation, and the heavy precipitation is on our flight path, or an Aviation Severe Weather Watch indicates heavy precipitation, and the heavy precipitation is on our flight path, or a SIGMET indicates heavy precipitation, and the heavy precipitation is on our flight path, or the on-board radar indicates heavy precipitation, and the heavy precipitation is on our flight path, or a tower report indicates heavy precipitation, and the heavy precipitation is on our flight path, or a Terminal Doppler Weather Radar indicates heavy precipitation, and the heavy precipitation is on our flight path, or a forward-looking system indicates heavy precipitation, and the heavy precipitation is on our flight path, or a flight crew observation indicates heavy precipitation on our flight path, THEN there are indications of heavy precipitation on our flight path. 
IF ATIS information indicates heavy precipitation, and the heavy precipitation is near but not on on our flight path, or an Aviation Severe Weather Watch indicates heavy precipitation, and the heavy precipitation is near but not on our flight path, or a SIGMET indicates heavy precipitation, and the heavy precipitation is near but not on our flight path, or the on-board radar indicates heavy precipitation, and the heavy precipitation is near but not on our flight path, or a tower report indicates heavy precipitation, and the heavy precipitation is near but not on our flight path, or a Terminal Doppler Weather Radar indicates heavy precipitation, and the heavy precipitation is near but not on our flight path, or a forward-looking system indicates heavy precipitation, and the heavy precipitation is near but not on our flight path,

or a flight crew observation indicates heavy precipitation near our flight path, THEN there are indications of heavy precipitation near our flight path.

\section{RAINSHOWER RULEBASE}

IF ATIS information indicates a rain shower, and the rain shower is on our flight path, or an Aviation Severe Weather Watch indicates a rain shower, and the rain shower is on our flight path,

or a SIGMET indicates a rain shower, and the rain shower is on our flight path, or the on-board radar indicates a rain shower, and the rain shower is on our flight path,

or a tower report indicates a rain shower, and the rain shower is on our flight path, or a Terminal Doppler Weather Radar indicates a rain shower, and the rain shower is on our flight path, or a forward-looking system indicates a rain shower, and the rain shower is on our flight path, or a flight crew observation indicates a rain shower on our flight path, THEN there are indications of a rain shower on our flight path.

IF ATIS information indicates a rain shower, and the rain shower is near but not on on our flight path, or an Aviation Severe Weather Watch indicates a rain shower, and the rain shower is near but not on our flight path, or a SIGMET indicates a rain shower, 
and the rain shower is near but not on our flight path,

or the on-board radar indicates a rain shower, and the rain shower is near but not on our flight path,

or a tower report indicates a rain shower, and the rain shower is near but not on our flight path,

or a Terminal Doppler Weather Radar indicates a rain shower, and the rain shower is near but not on our flight path,

or a forward-looking system indicates a rain shower, and the rain shower is near but not on our flight path,

or a flight crew observation indicates a rain shower near our flight path,

THEN there are indications of a rain shower near our flight path.

\section{LIGHTNING RULEBASE}

IF ATIS information indicates lightning, and the lightning is on our flight path,

or an Aviation Severe Weather Watch indicates lightning, and the lightning is on our flight path,

or a SIGMET indicates lightning, and the lightning is on our flight path, or the Lightning Sensor System indicates lightning, and the lightning is on our flight path, or a tower report indicates lightning, and the lightning is on our flight path, or a forward-looking system indicates lightning, and the lightning is on our flight path, or a flight crew observation indicates lightning on our flight path, THEN there are indications of lightning on our flight path.

IF ATIS information indicates lightning, and the lightning is near but not on on our flight path, or an Aviation Severe Weather Watch indicates lightning, and the lightning is near but not on our flight path, or a SIGMET indicates lightning, and the lightning is near but not on our flight path, or the Lightning Sensor System indicates lightning, and the lightning is near but not on our flight path, or a tower report indicates lightning, and the lightning is near but not on our flight path, or a forward-looking system indicates lightning, and the lightning is near but not on our flight path, or a flight crew observation indicates lightning near our flight path, 
THEN there are indications of lightning near our flight path.

\section{VIRGA RULEBASE}

IF ATIS information indicates virga, and the virga is on our flight path, or an Aviation Severe Weather Watch indicates virga, and the virga is on our flight path, or a SIGMET indicates virga, and the virga is on our flight path,

or a tower report indicates virga, and the virga is on our flight path,

or a forward-looking system indicates virga, and the virga is on our flight path,

or a flight crew observation indicates virga on our flight path, THEN there are indications of virga on our flight path.

IF ATIS information indicates virga, and the virga is near but not on on our flight path, or an Aviation Severe Weather Watch indicates virga, and the virga is near but not on our flight path,

or a SIGMET indicates virga, and the virga is near but not on our flight path, or a tower report indicates virga, and the virga is near but not on our flight path, or a forward-looking system indicates virga, and the virga is near but not on our flight path, or a flight crew observation indicates virga near our flight path, THEN there are indications of virga near our flight path.

\section{TURBULENCE RULEBASE}

IF ATIS information indicates moderate or greater turbulence, and the turbulence is on our flight path,

or an Aviation Severe Weather Watch indicates moderate or greater turbulence, and the turbulence is on our flight path,

or a SIGMET indicates moderate or greater turbulence, and the turbulence is on our flight path, or the on-board radar indicates turbulence, and the turbulence is on our flight path, or a tower report indicates turbulence, 
and the turbulence is on our flight path,

or a Terminal Doppler Weather Radar indicates moderate or greater turbulence, and the turbulence is on our flight path,

or a wind profiler indicates moderate or greater turbulence, and the turbulence is on our flight path,

or a forward-looking system indicates moderate or greater turbulence, and the turbulence is on our flight path,

or a flight crew observation indicates moderate or greater turbulence on our flight path,

THEN there are indications of moderate or greater turbulence on our flight path.

IF ATIS information indicates moderate or greater turbulence, and the turbulence is near but not on on our flight path, or an Aviation Severe Weather Watch indicates moderate or greater turbulence, and the turbulence is near but not on our flight path, or a SIGMET indicates moderate or greater turbulence, and the turbulence is near but not on our flight path, or the on-board radar indicates turbulence, and the turbulence is near but not on our flight path, or a tower report indicates turbulence, and the turbulence is near but not on our flight path, or a Terminal Doppler Weather Radar indicates moderate or greater turbulence, and the turbulence is near but not on our flight path, or a wind profiler indicates moderate or greater turbulence, and the turbulence is near but not on our flight path, or a forward-looking system indicates moderate or greater turbulence, and the turbulence is near but not on our flight path, or a flight crew observation indicates turbulence near our flight path, THEN there are indications of moderate or greater turbulence near our flight path. 
Appendix C

\section{LISP Code for Rule Base Executive Functions}




\section{ORIGHSAL FAIS TS
OQ ROOW QUALITY}

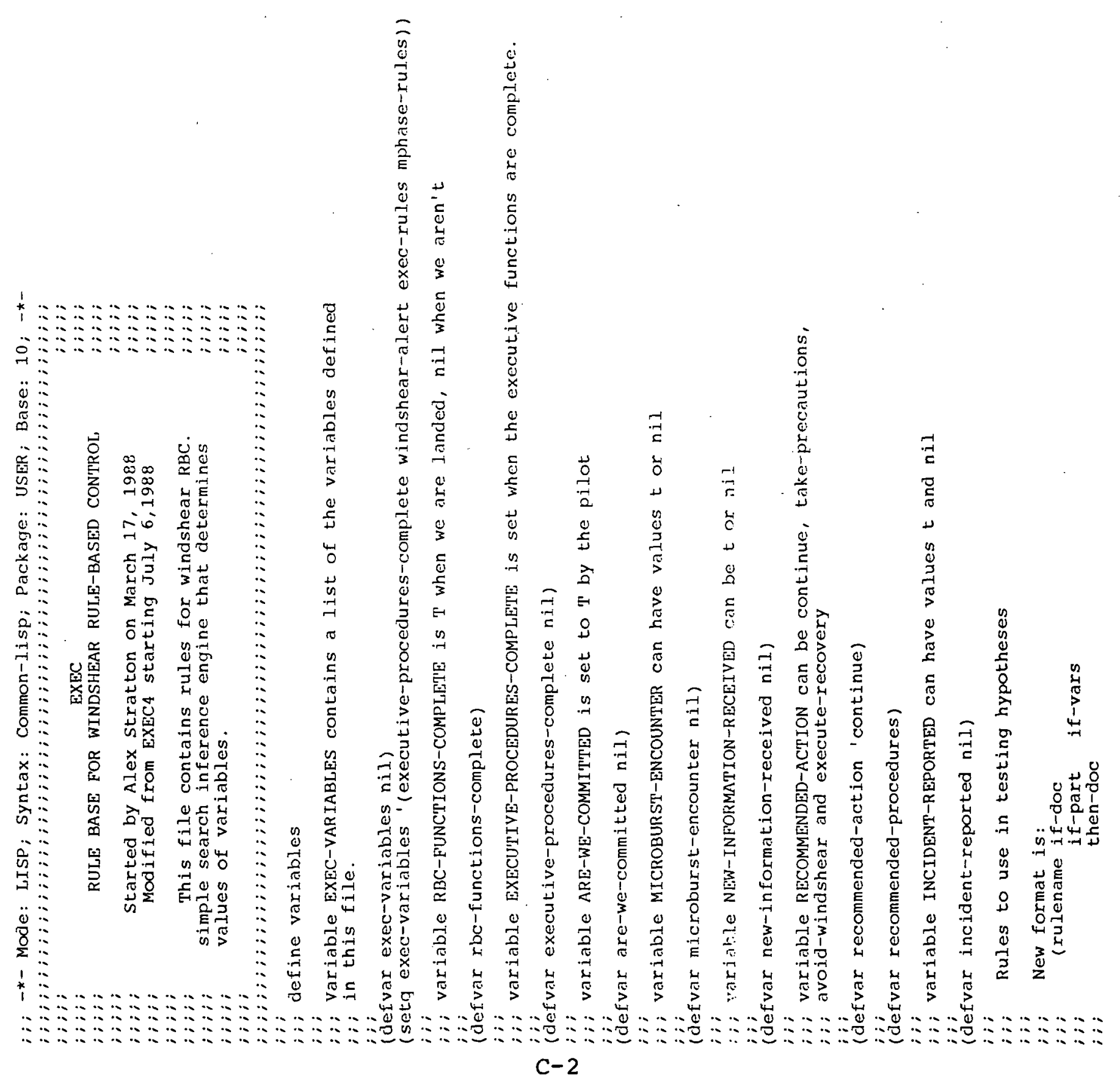




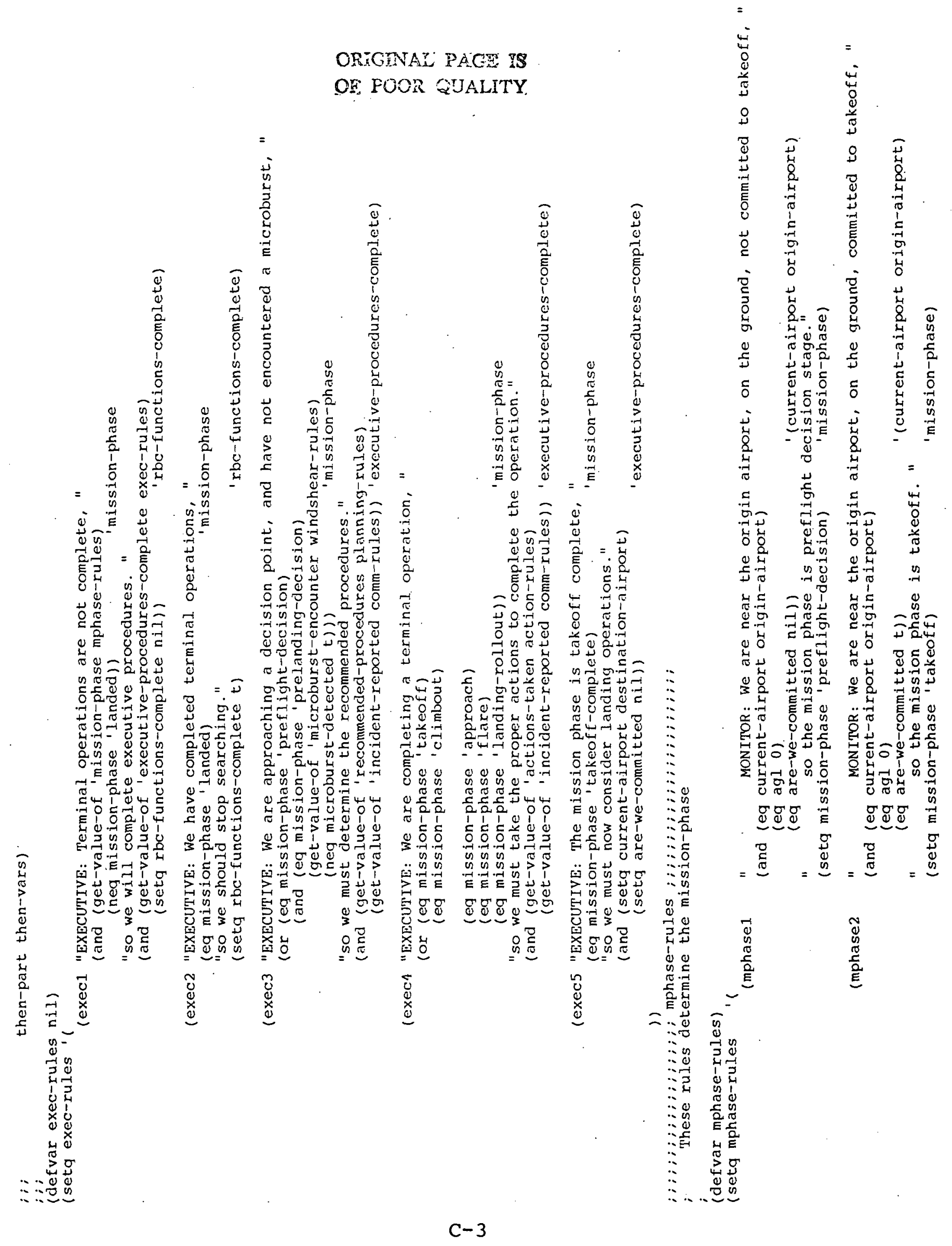




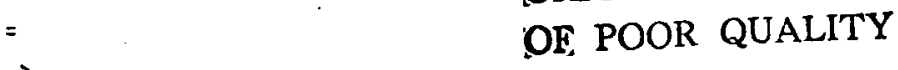

OF POOR QUALITY

ב⿱艹

至出

i

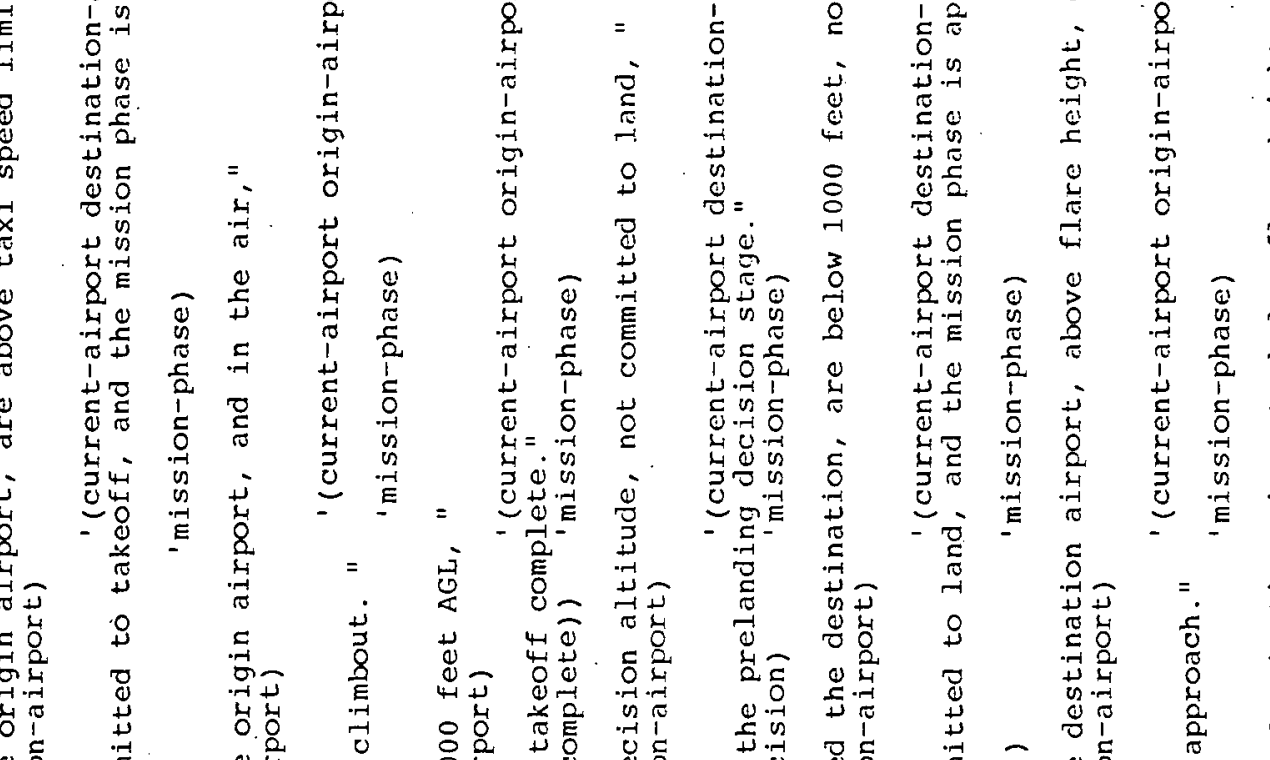

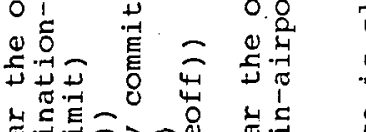

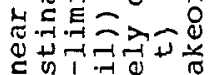

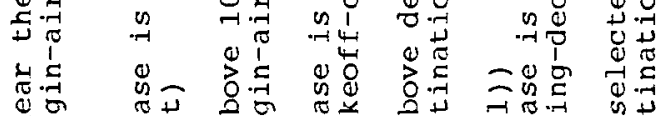

हี

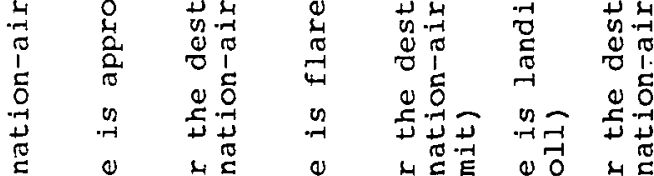

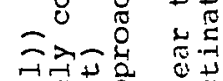

o

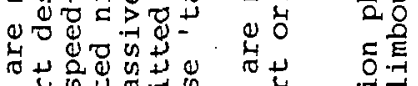

क्ष

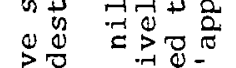

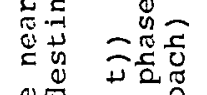

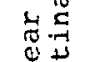

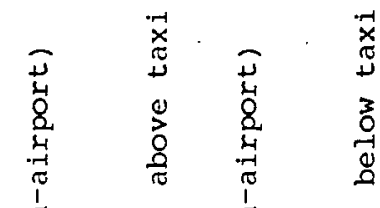

$\begin{array}{ccc}1 & 1 \\ 0 & 0 & 5 \\ 0 & 5 & 0 \\ 0 & 0 & 0\end{array}$

मे कू

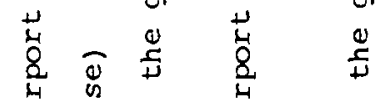

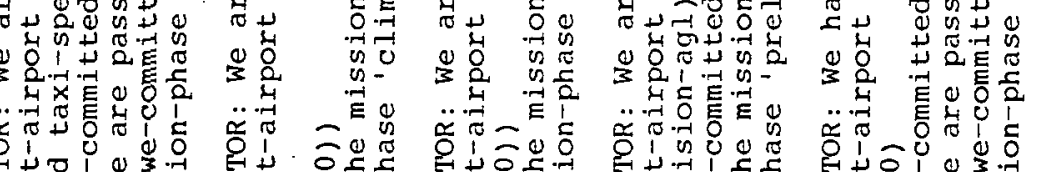

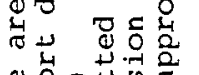

(1)

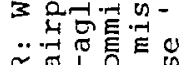

$=0 \begin{aligned} & 0 \\ & 0\end{aligned}$

$=$ ข 1 त

4 वृ

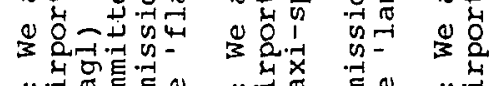

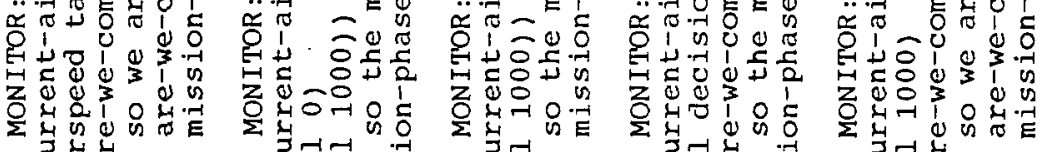

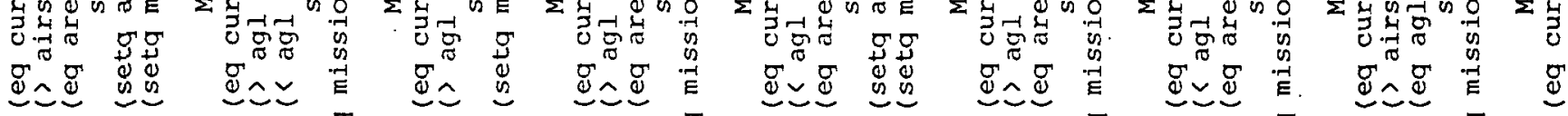

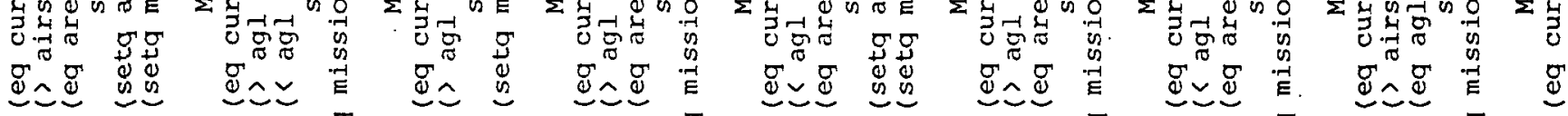

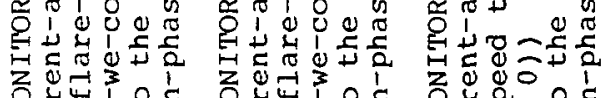

政政

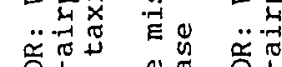

₹

之o

空嵌

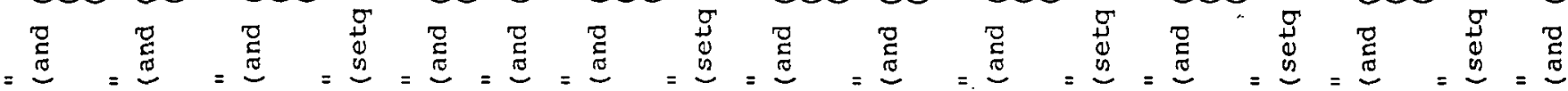

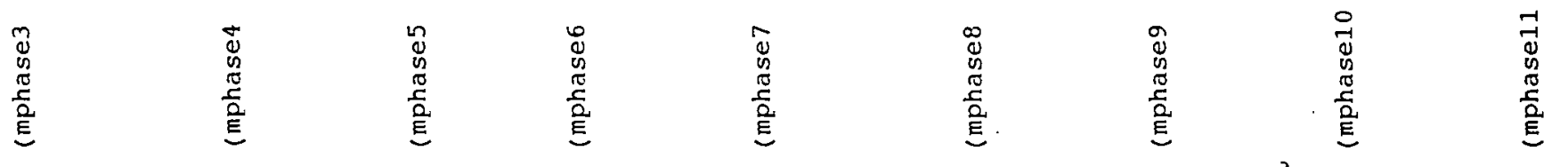




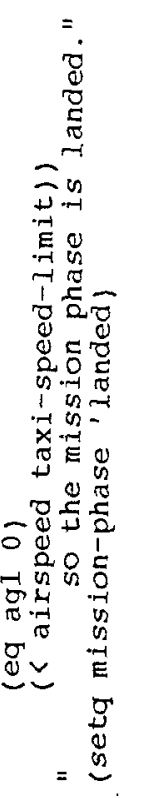


Appendix D

\section{LISP Code for Rules of Rule-Based Controller}


ORIGINAC PAGE IS

OF POOR QUALITY:

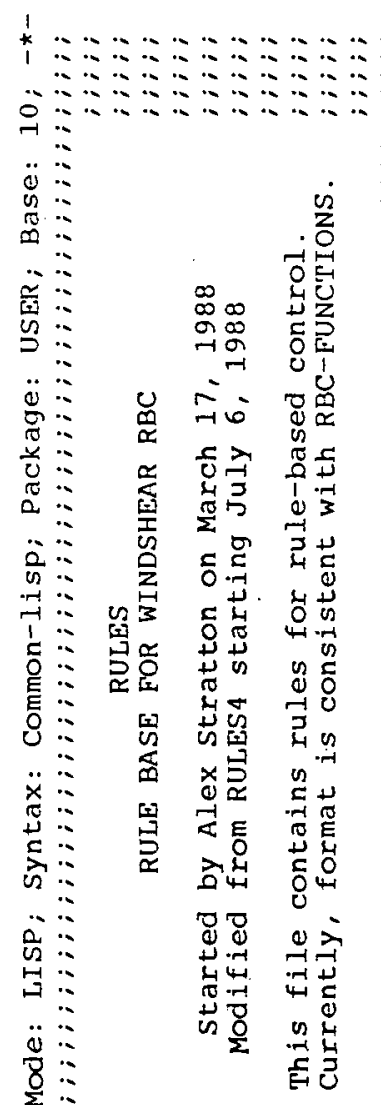

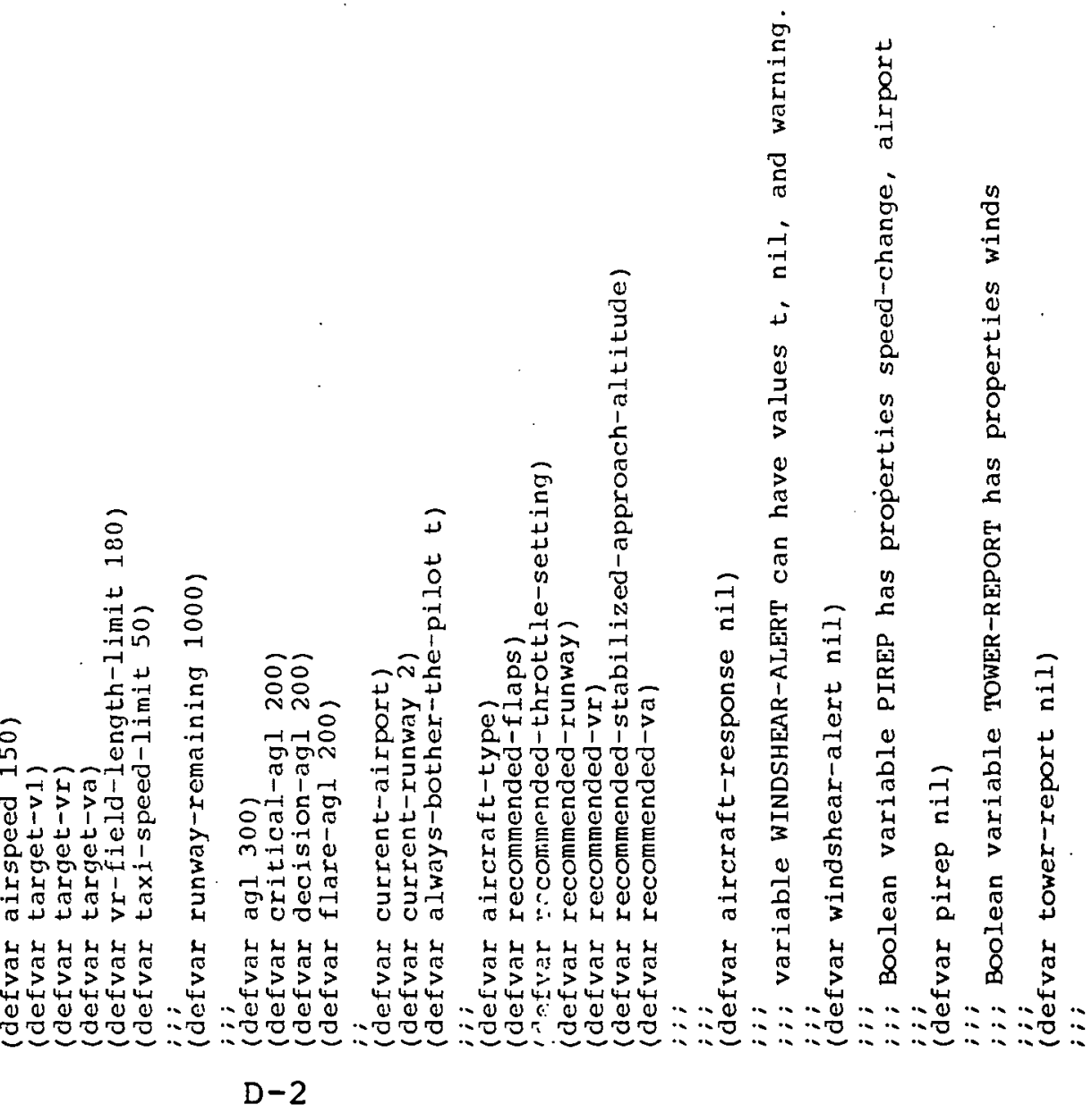


ORIGINAL PAGE IS
OF POOR QUALITY

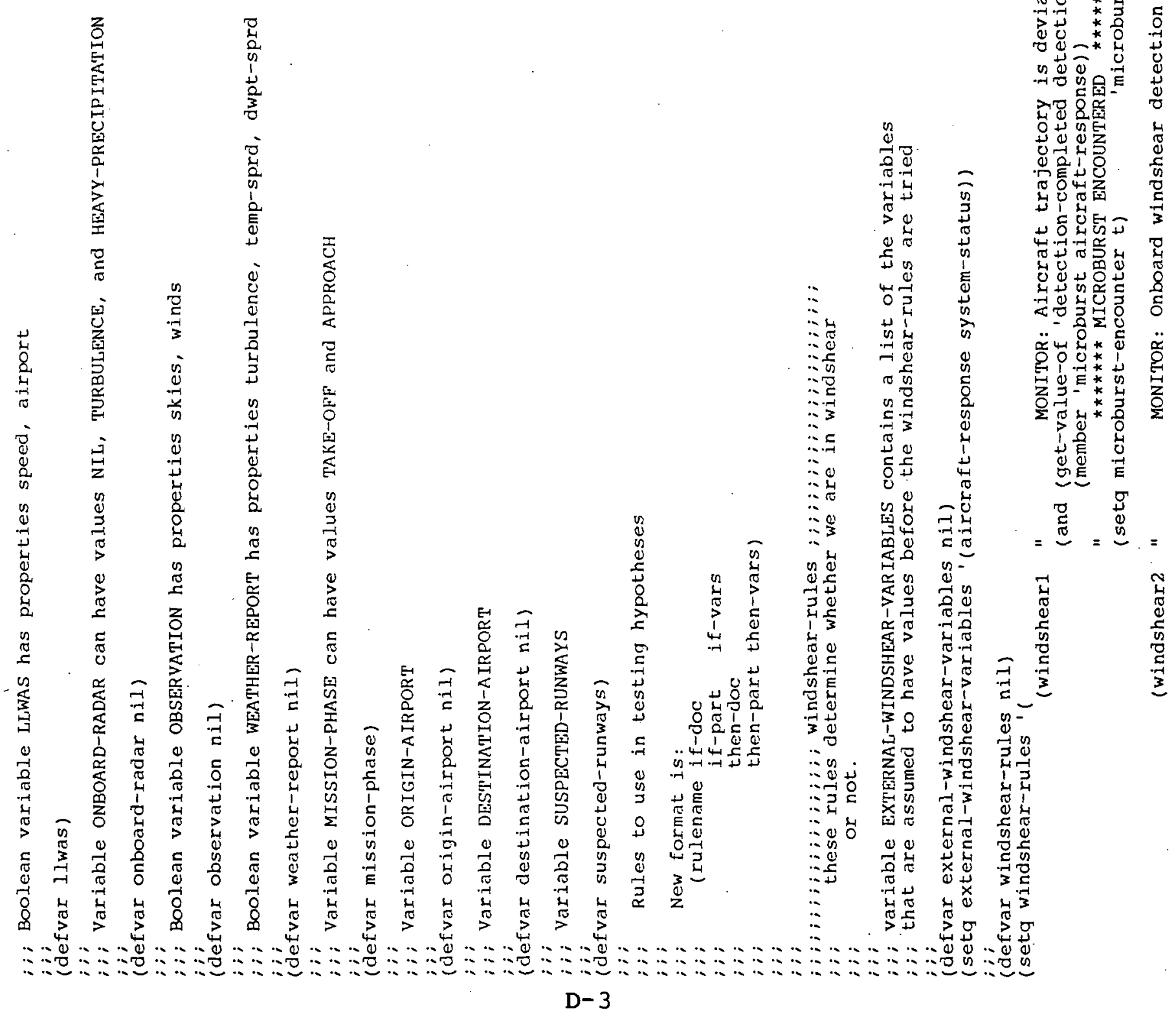



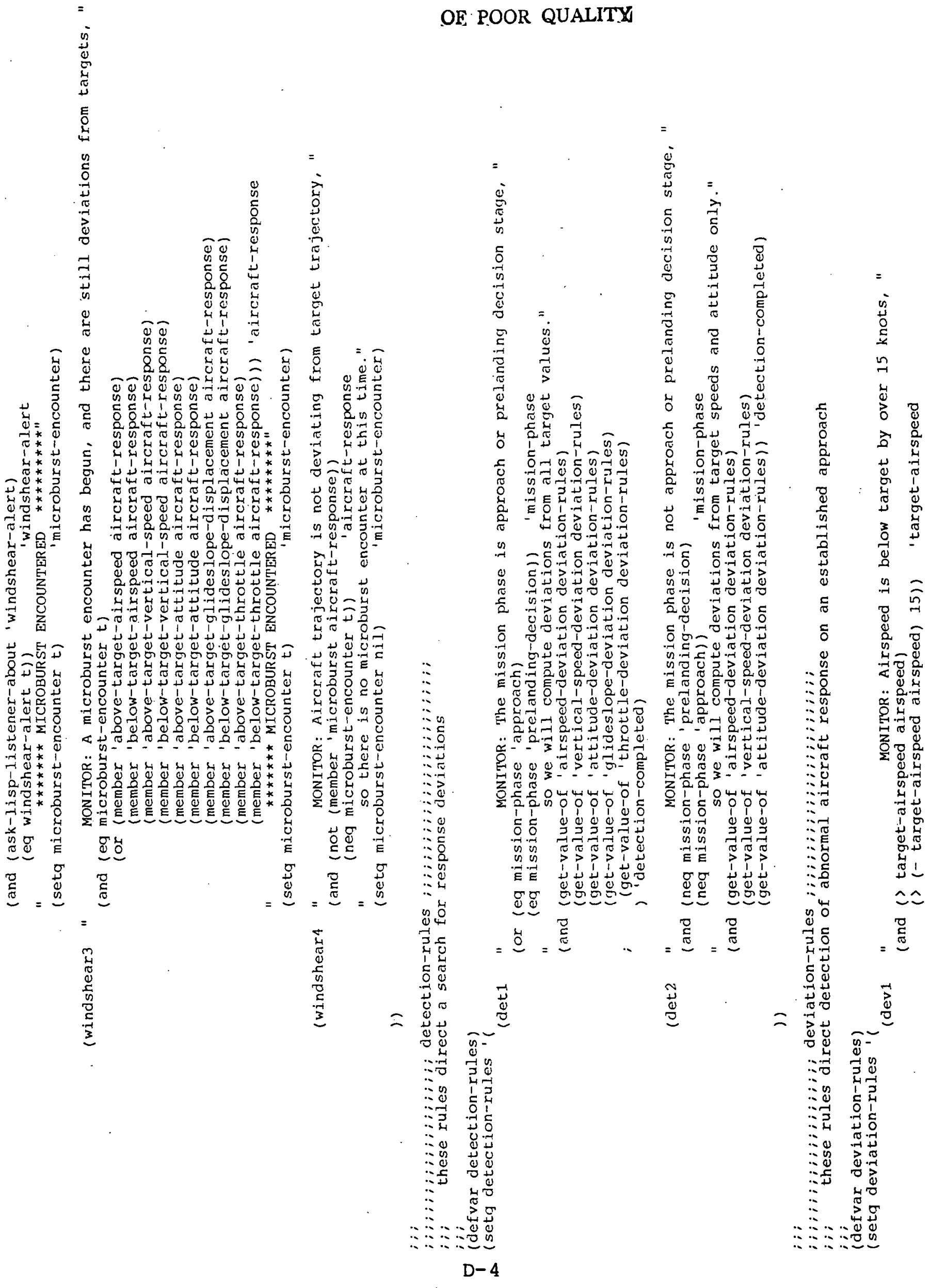


\section{ORIGIVAL PAGIS IS
OF POOR QUALITY}

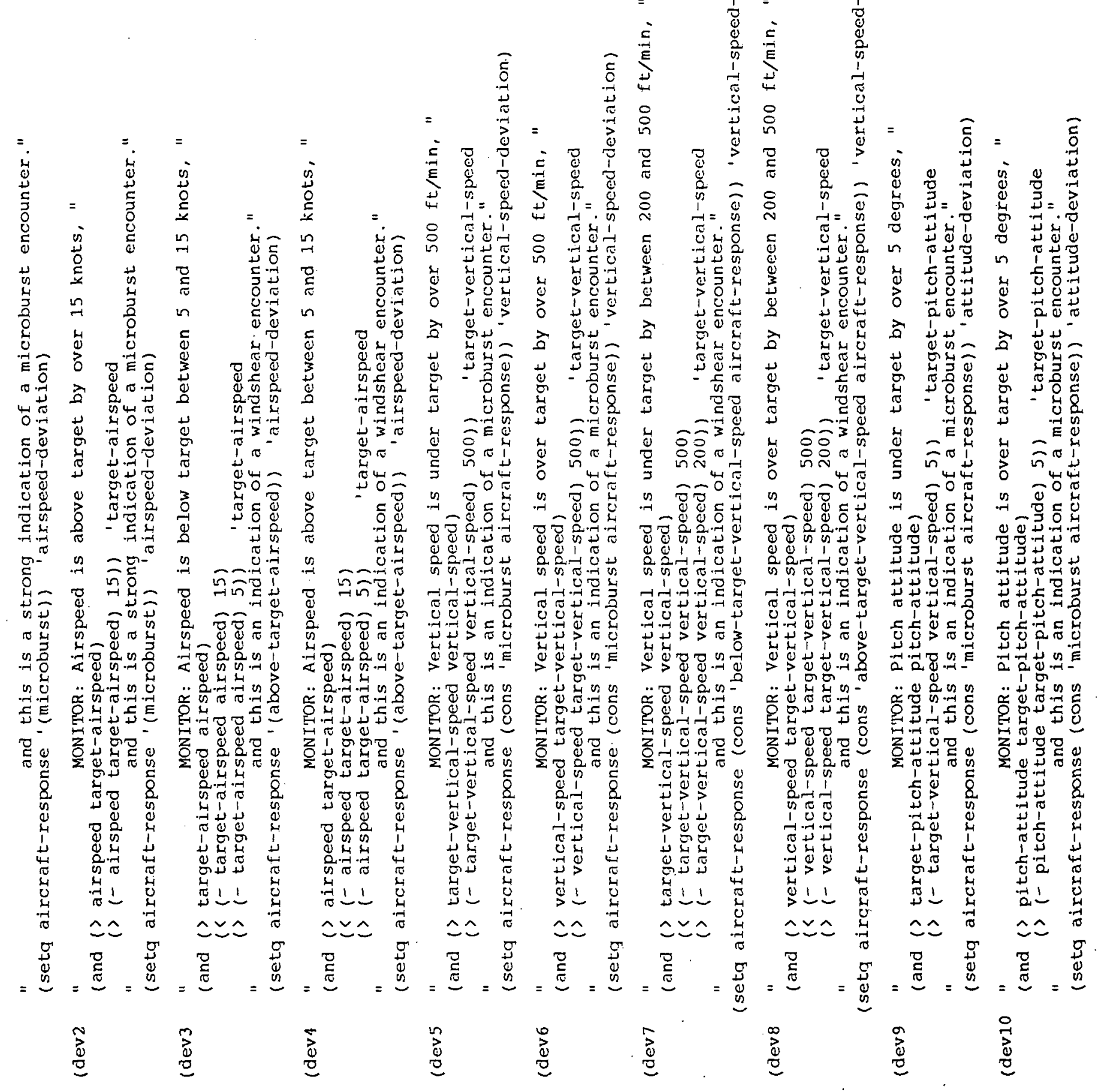




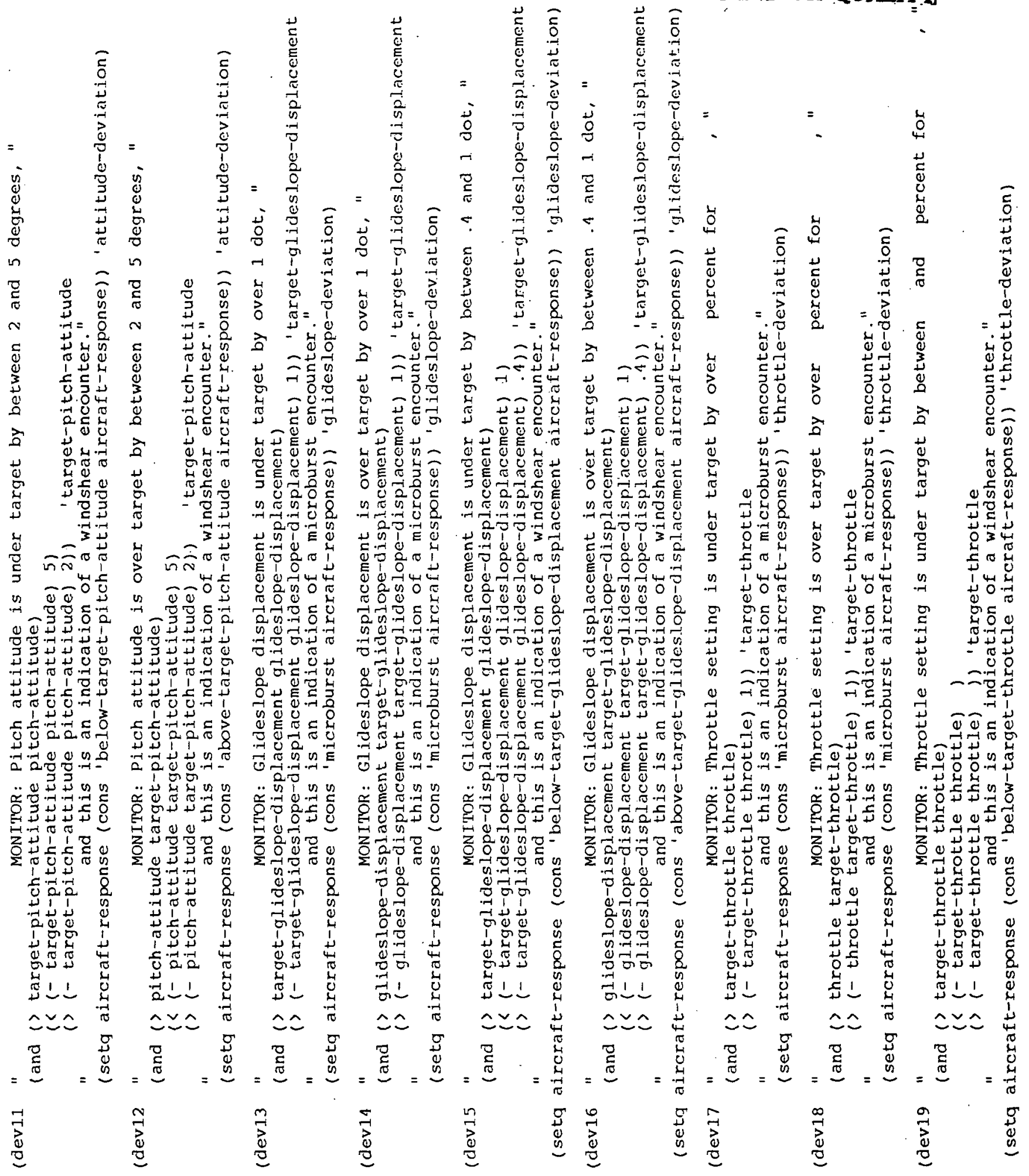




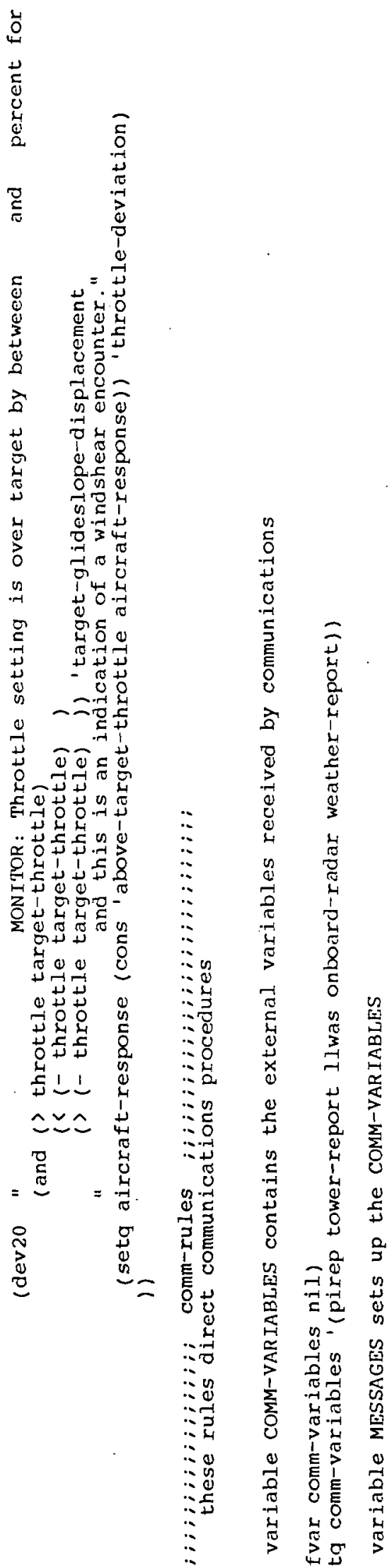

峁

ORIGHAAC PAGE

OF PCOR QUALITY

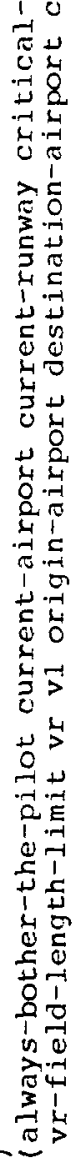

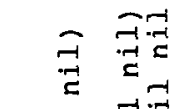

=-1 $=7$

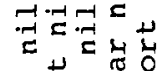

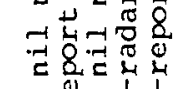

ह

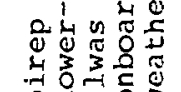

논도

y

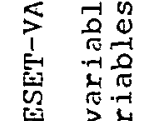

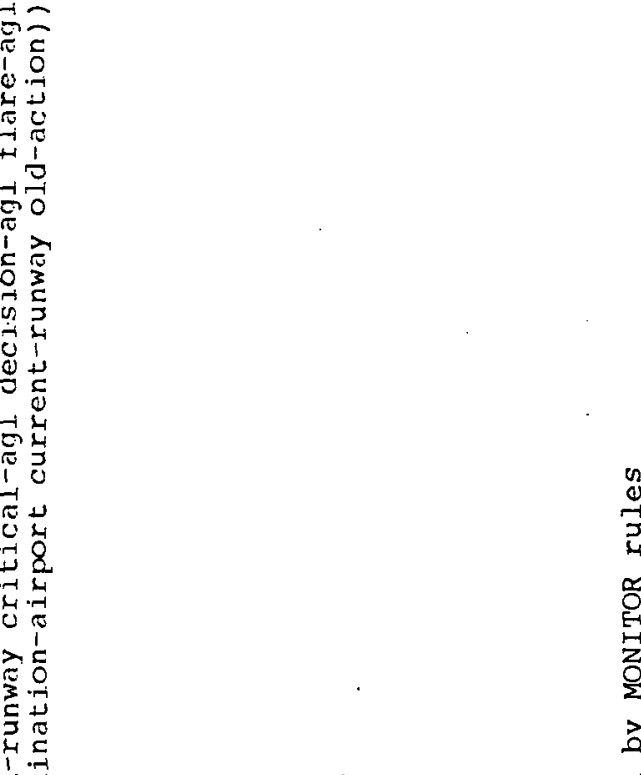

俩

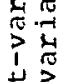

जी

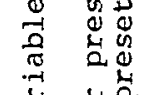

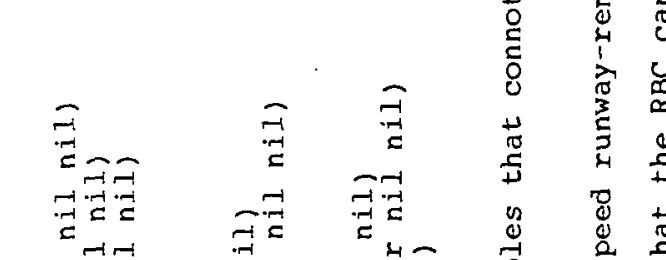

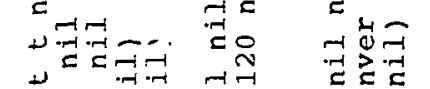

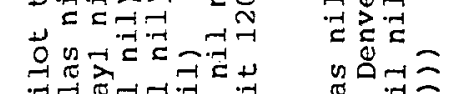
a

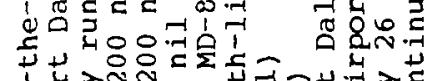

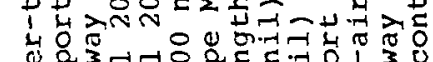

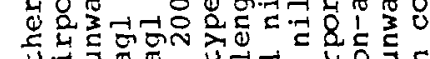

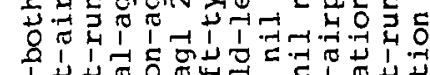
论范。

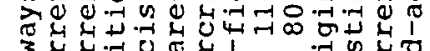

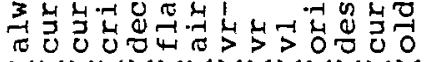
$\widehat{x}=$ o 过 章 峞

$\frac{4}{2}$ 

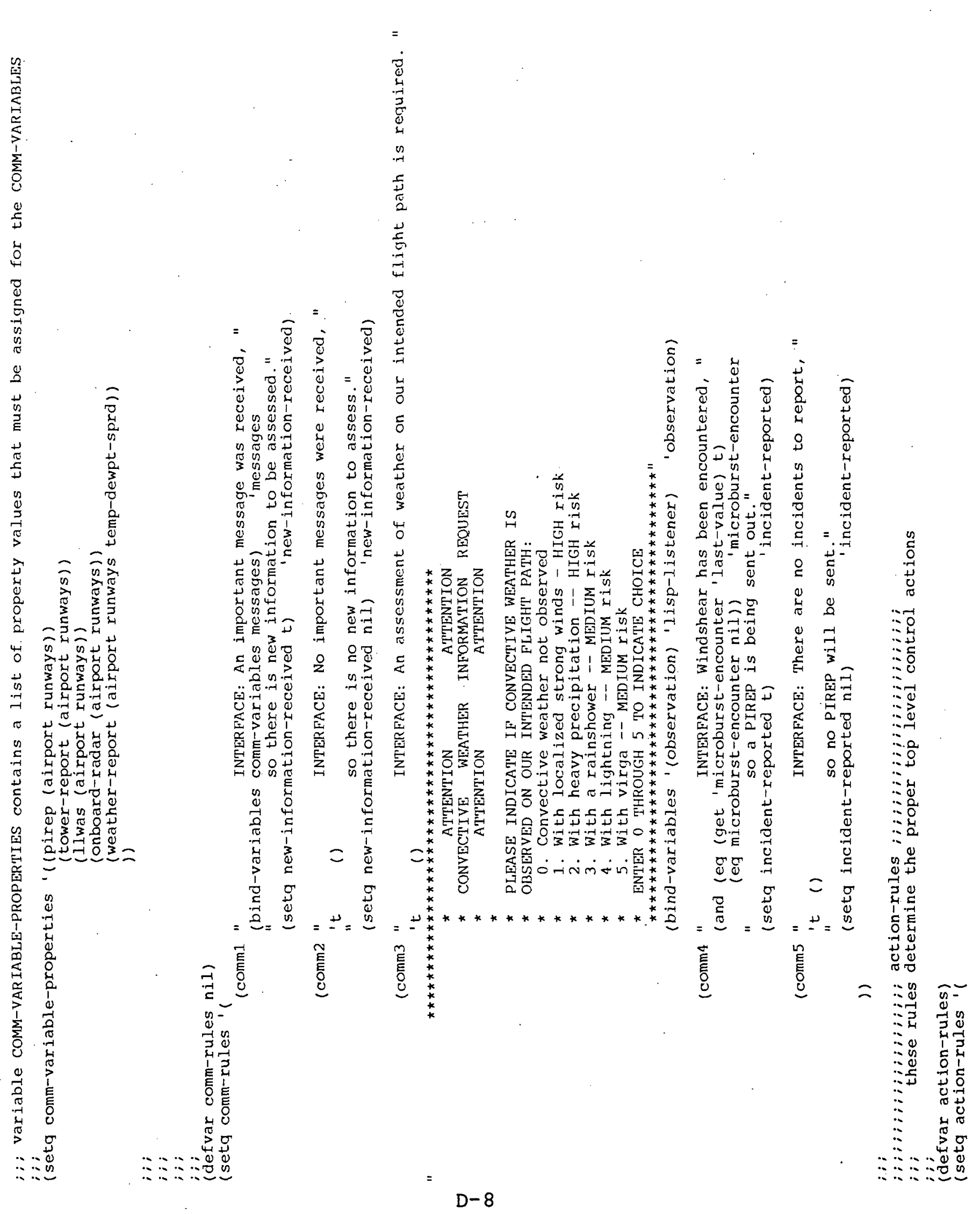


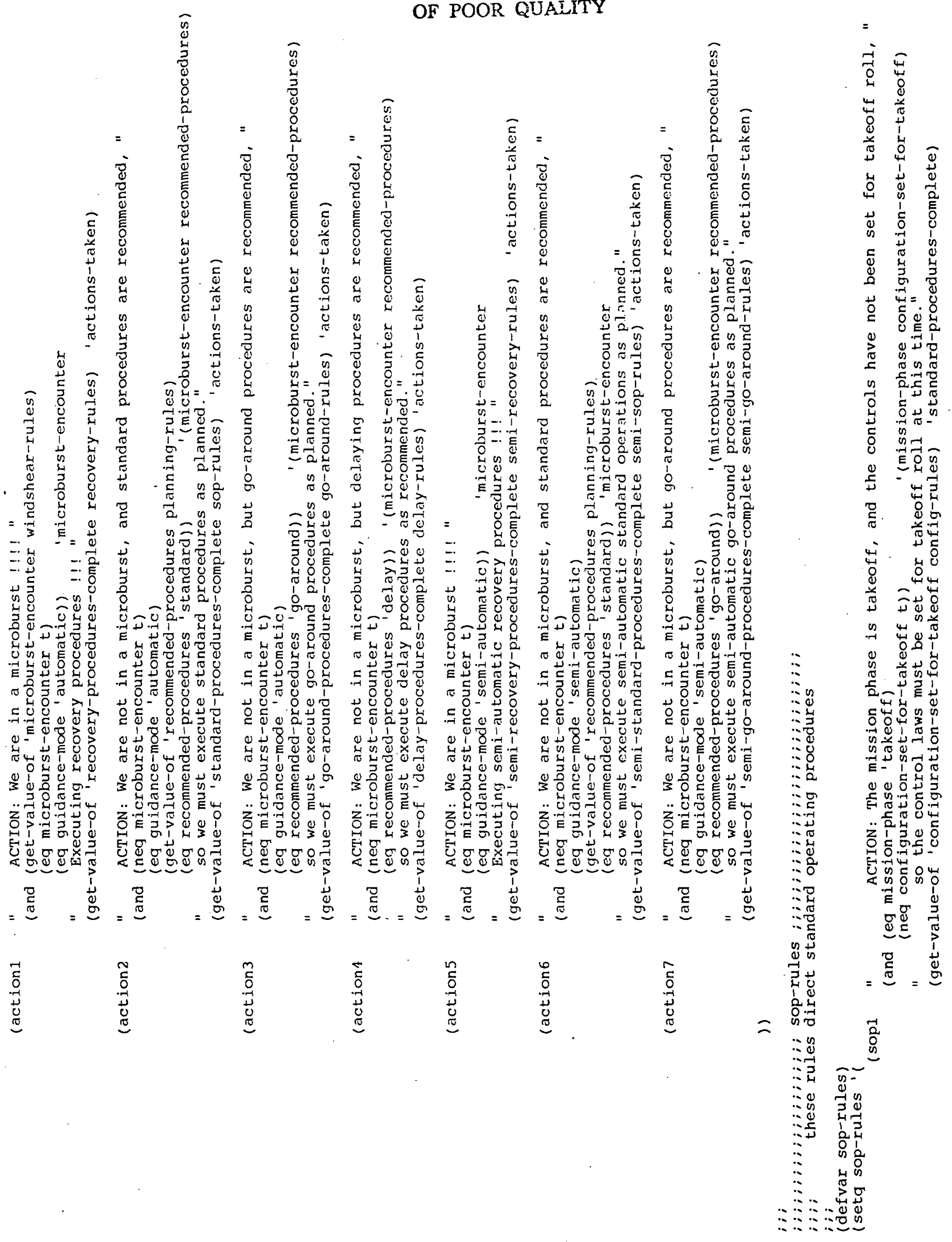




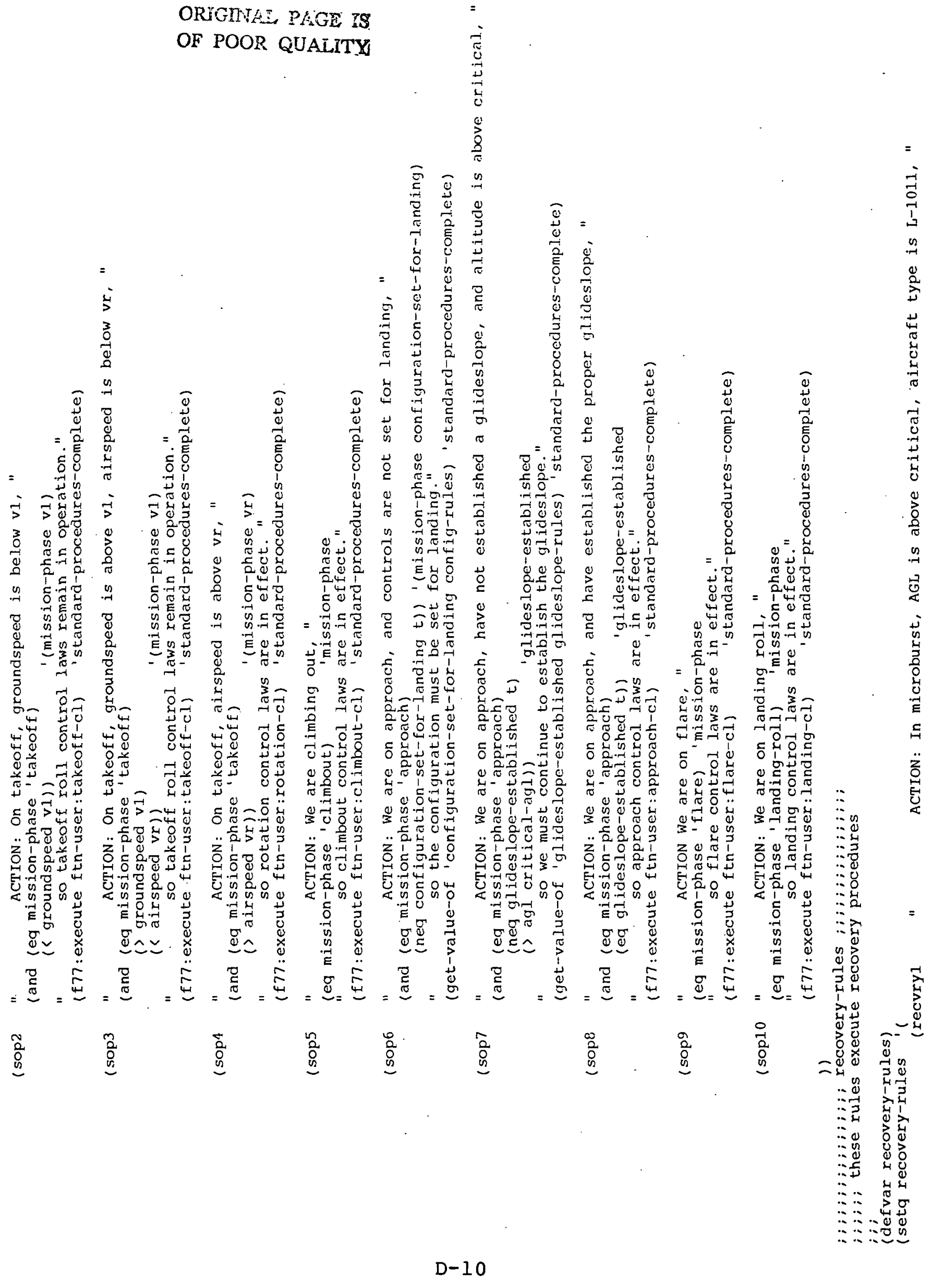




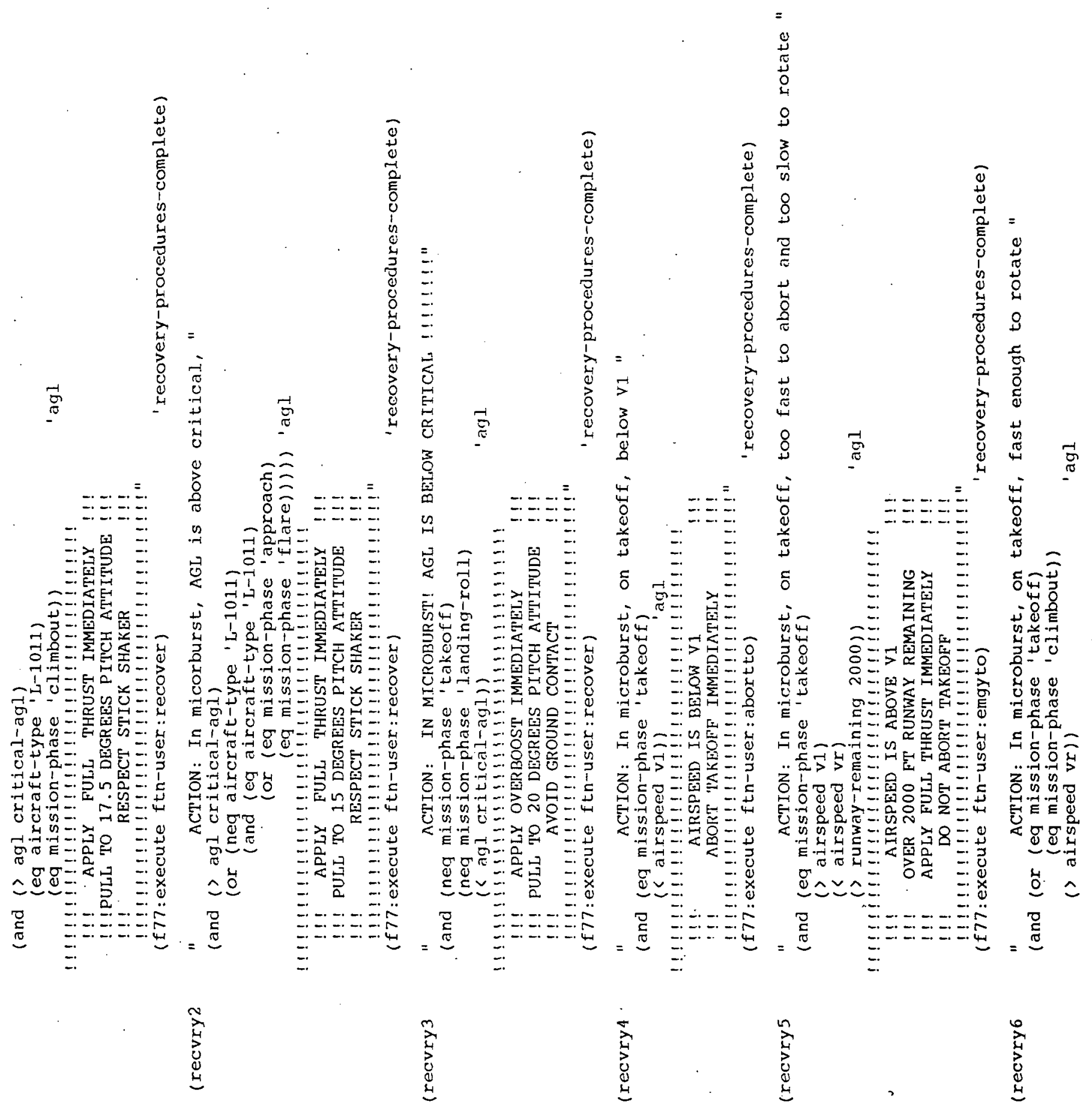




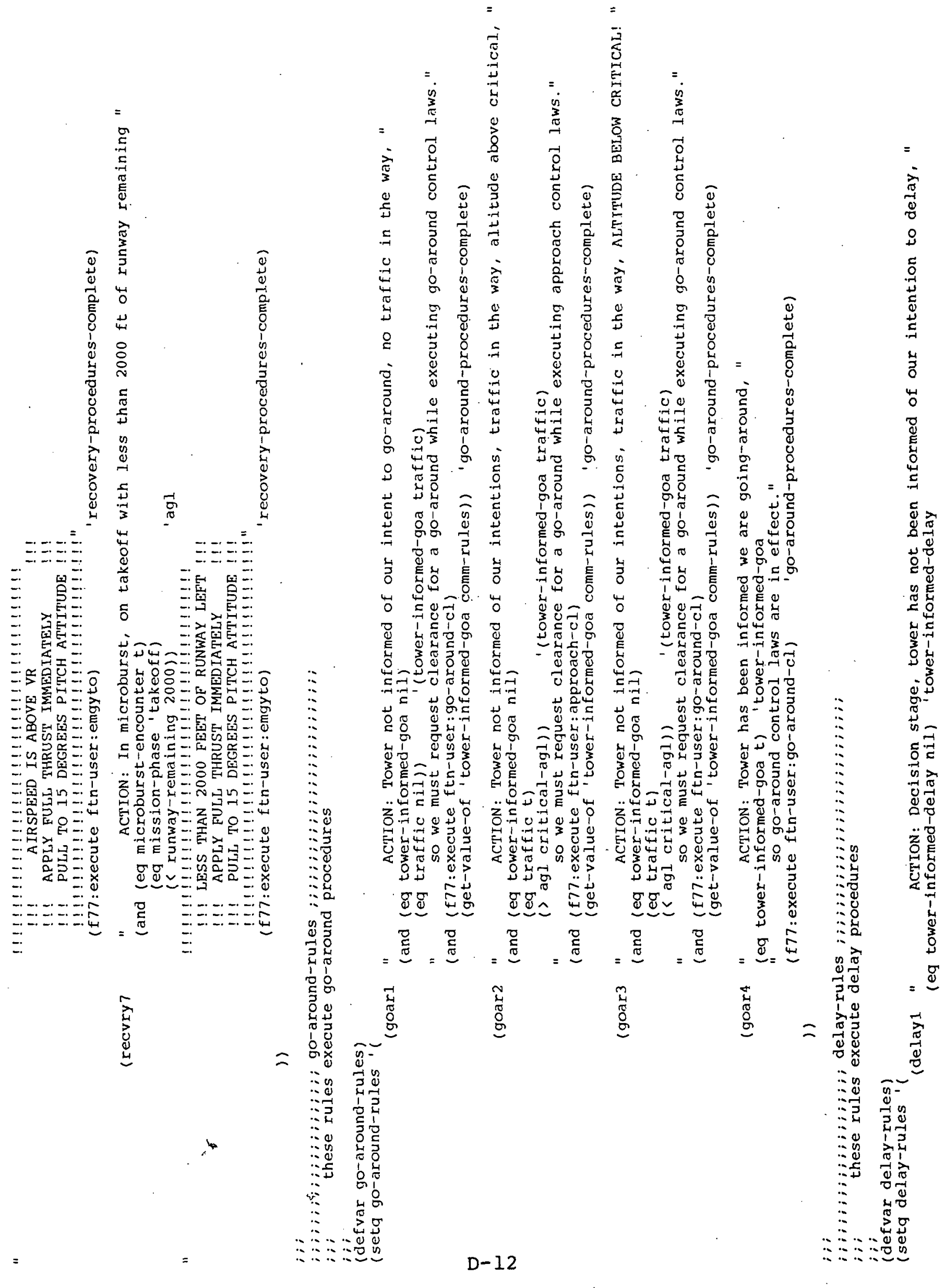




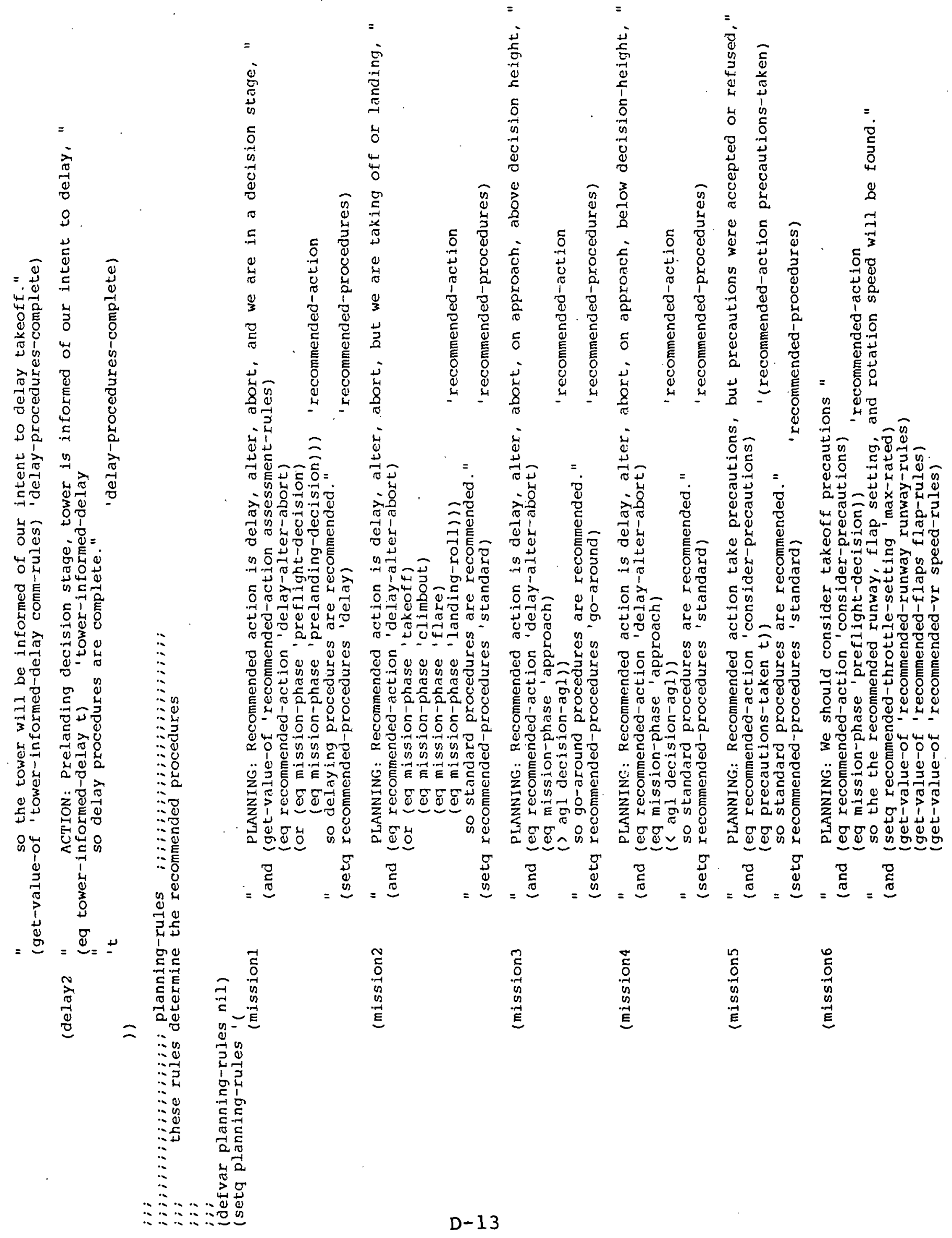




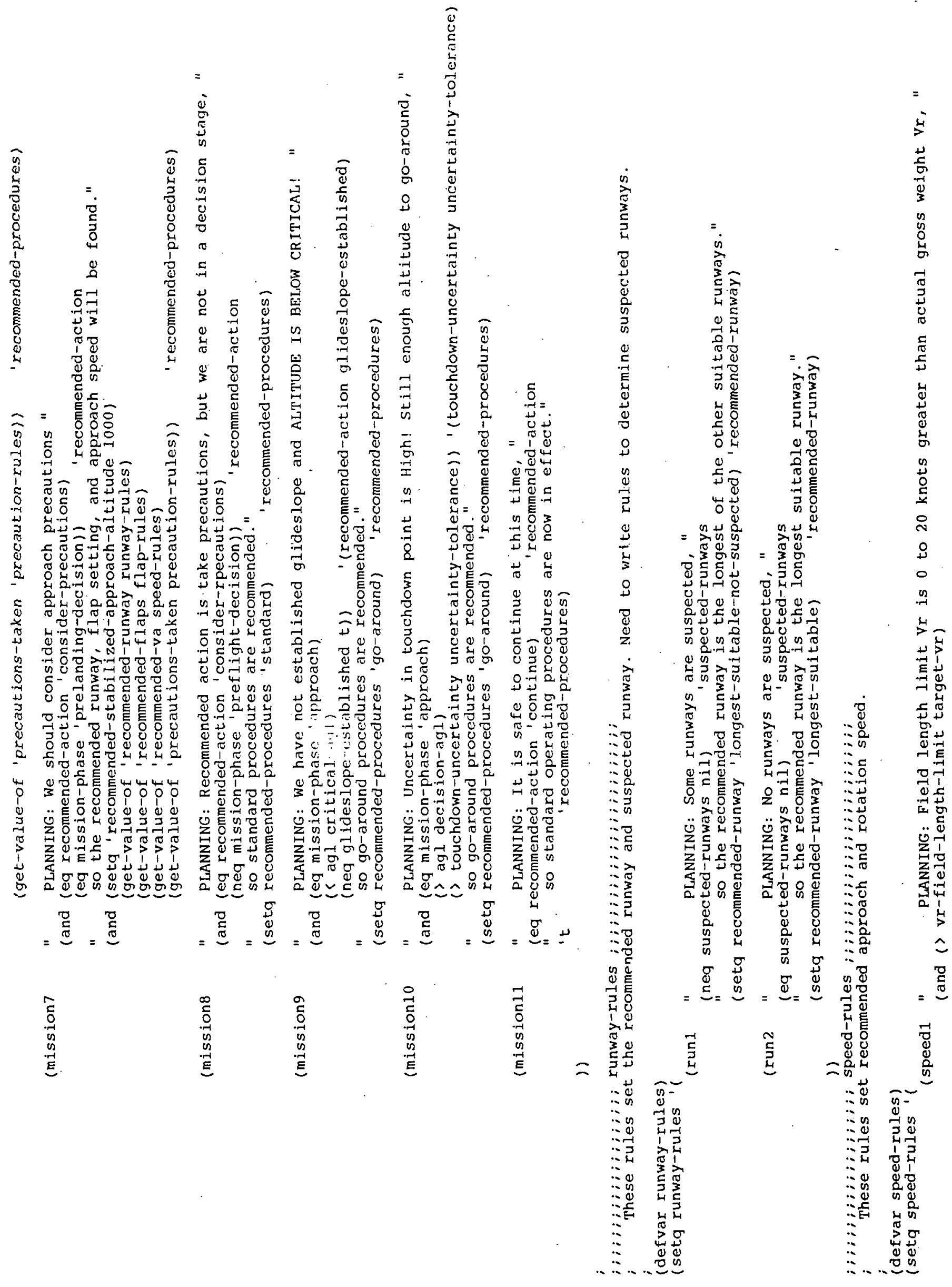




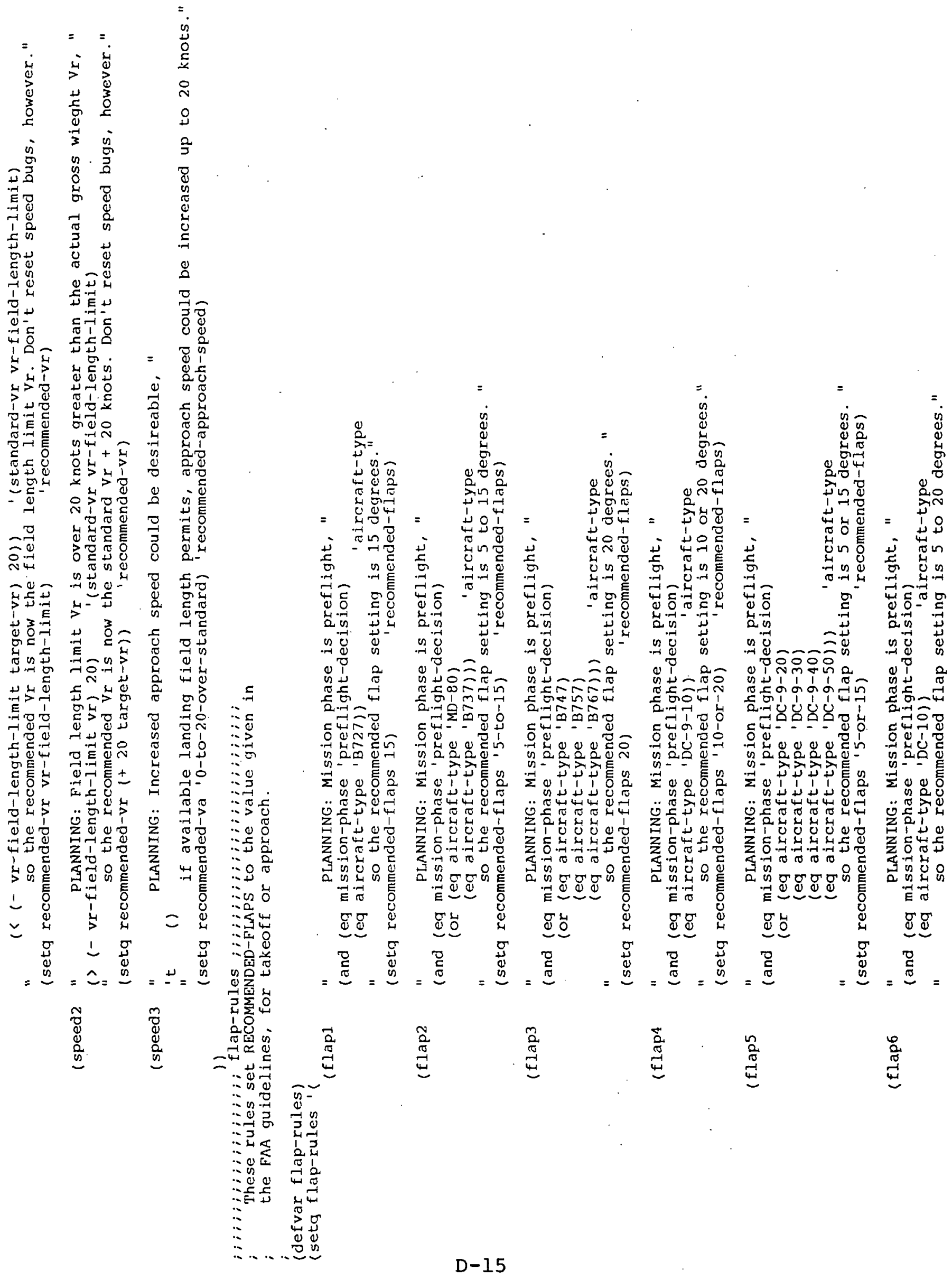




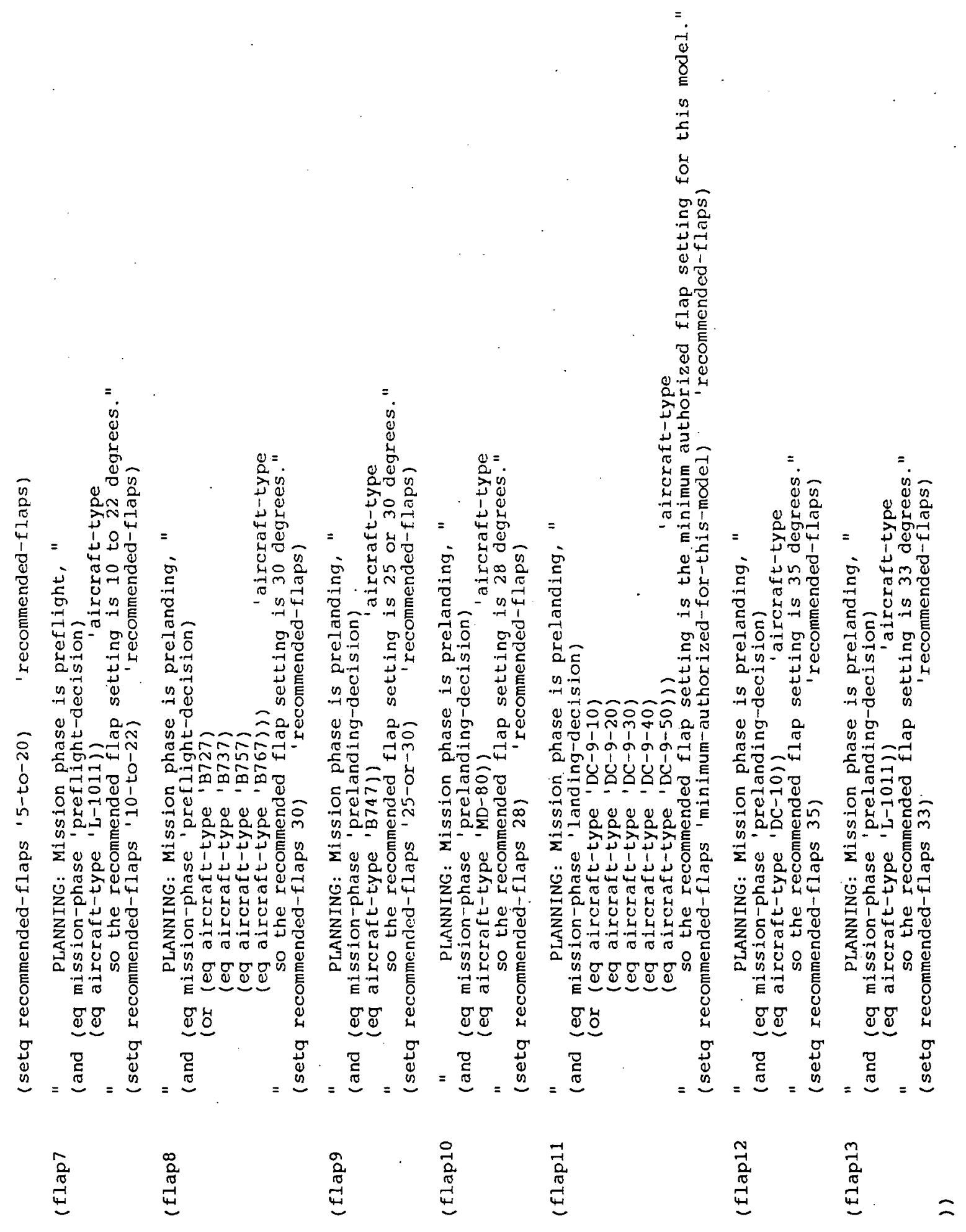




\section{Appendix E \\ LISP Code for Rules of Wind Shear Assessment}

$$
\text { E-1 }
$$




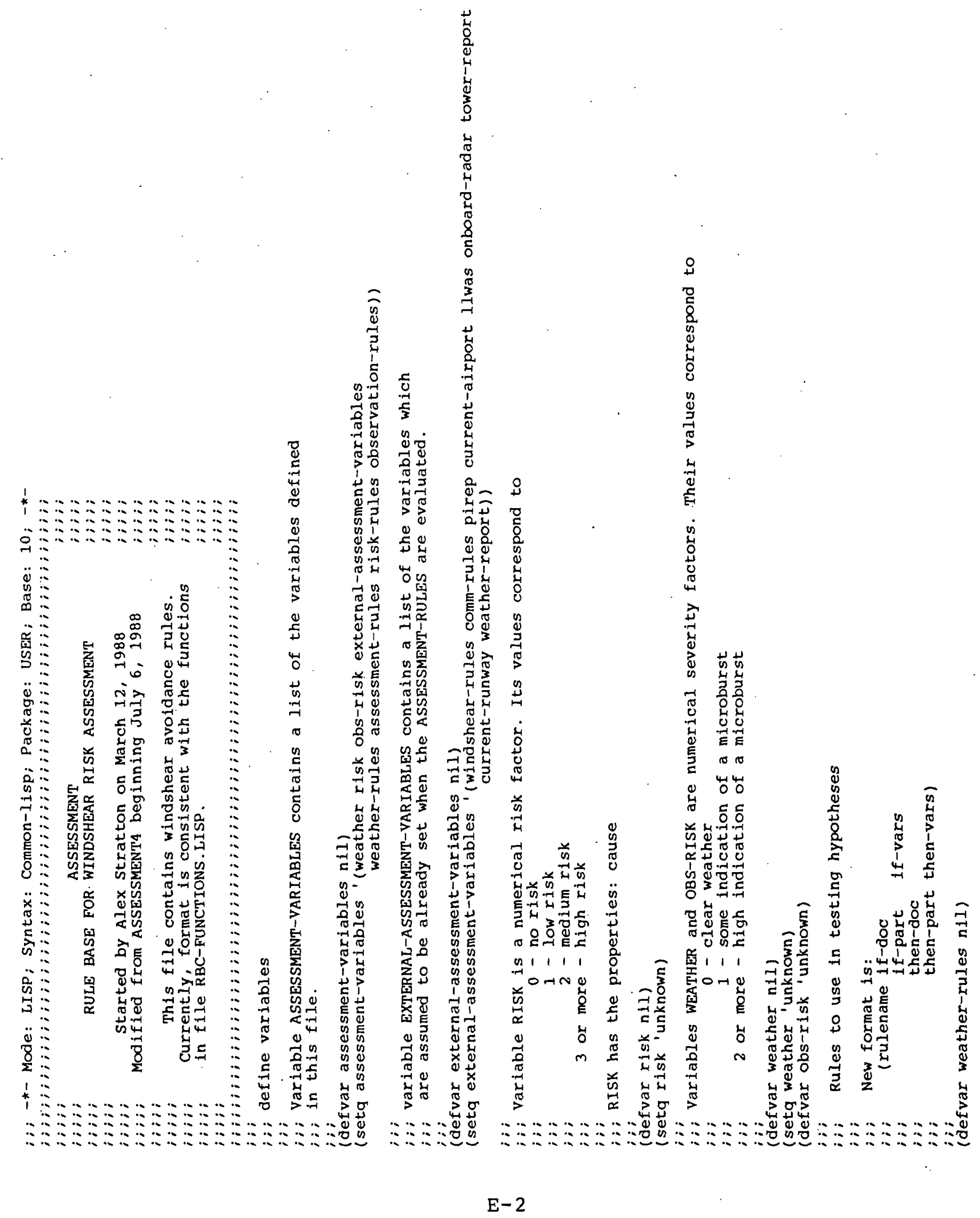




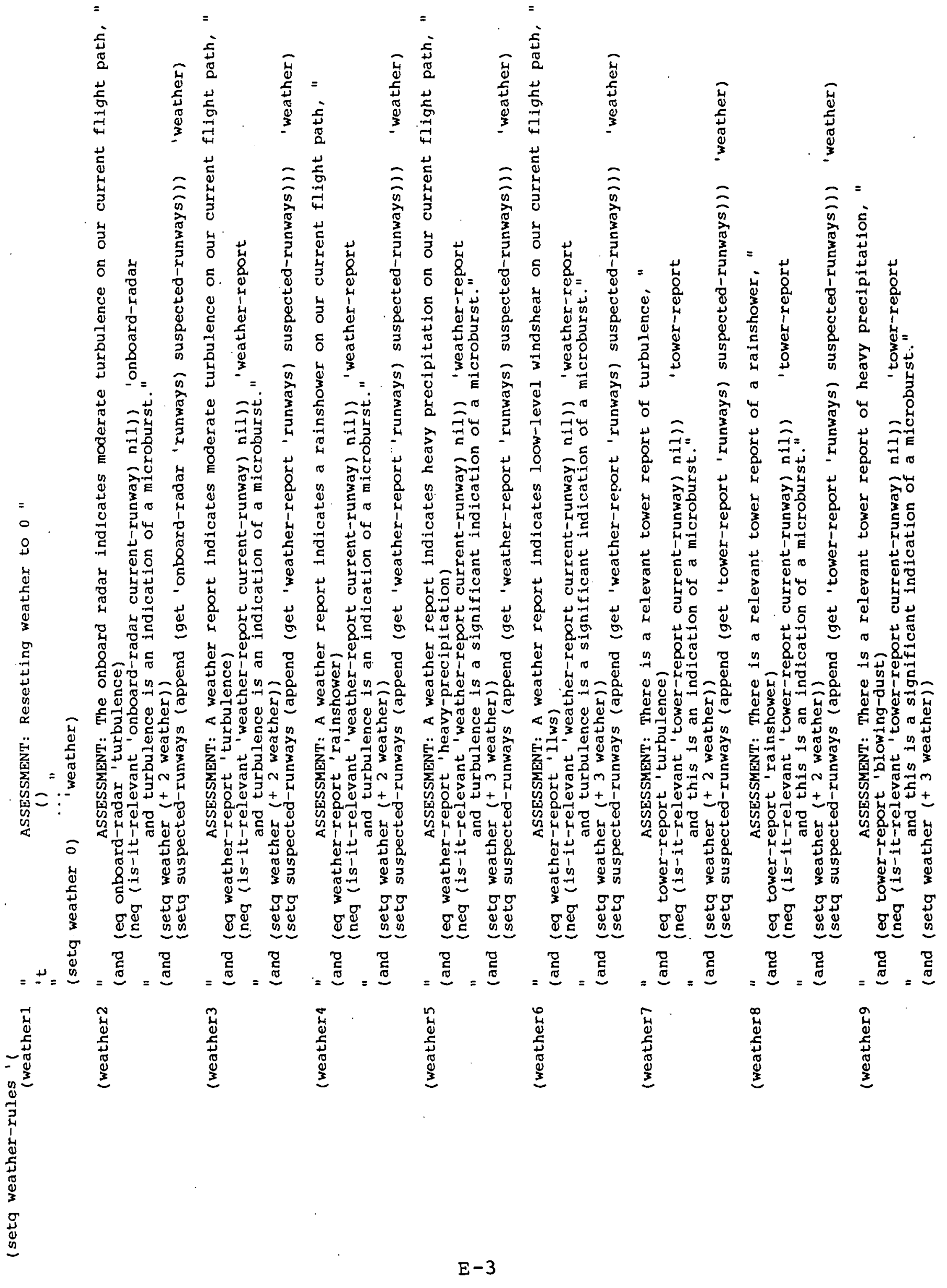




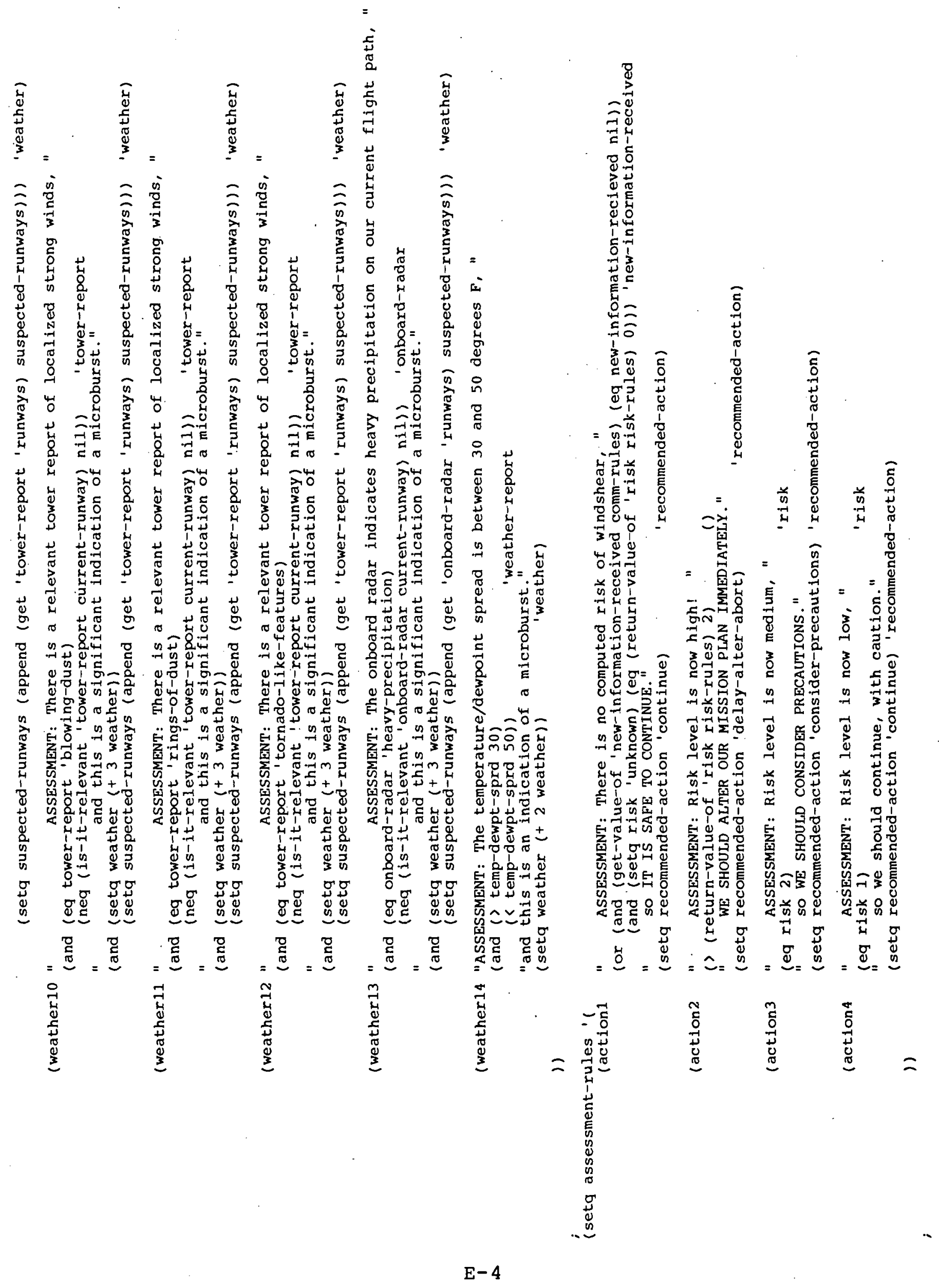




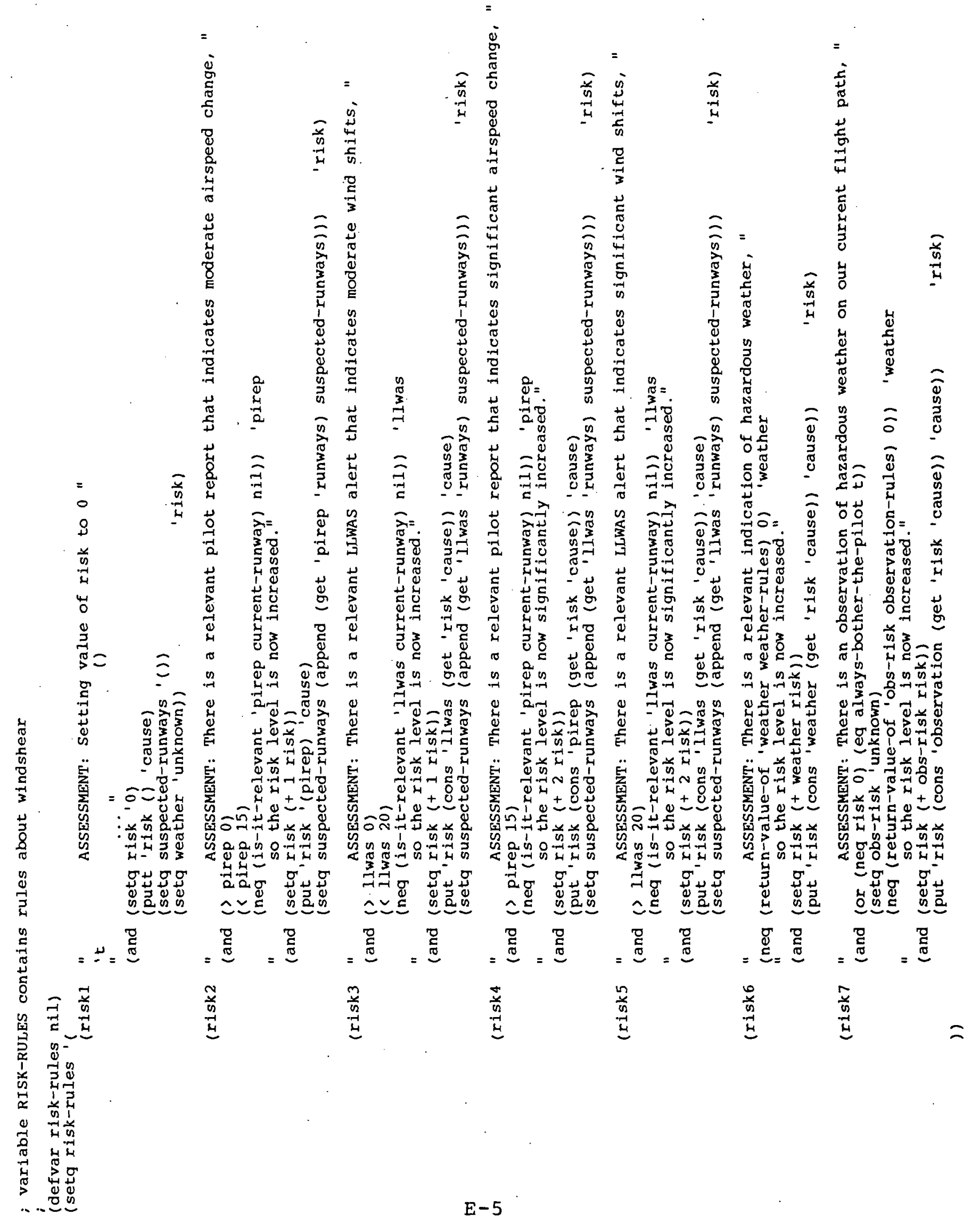




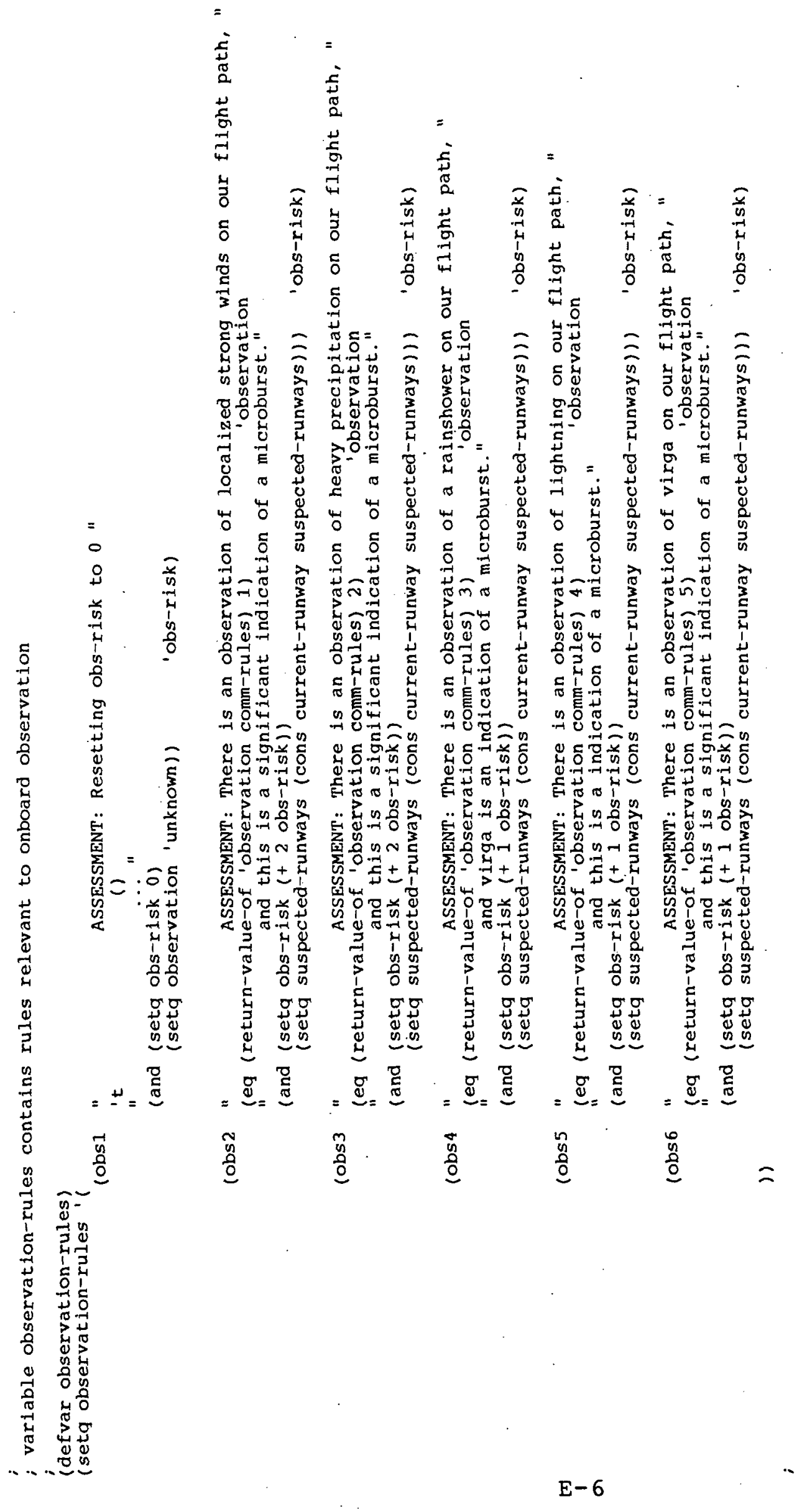




$$
\begin{gathered}
\text { Appendix } F \\
\text { LISP Code for Rule Base Control Functions }
\end{gathered}
$$




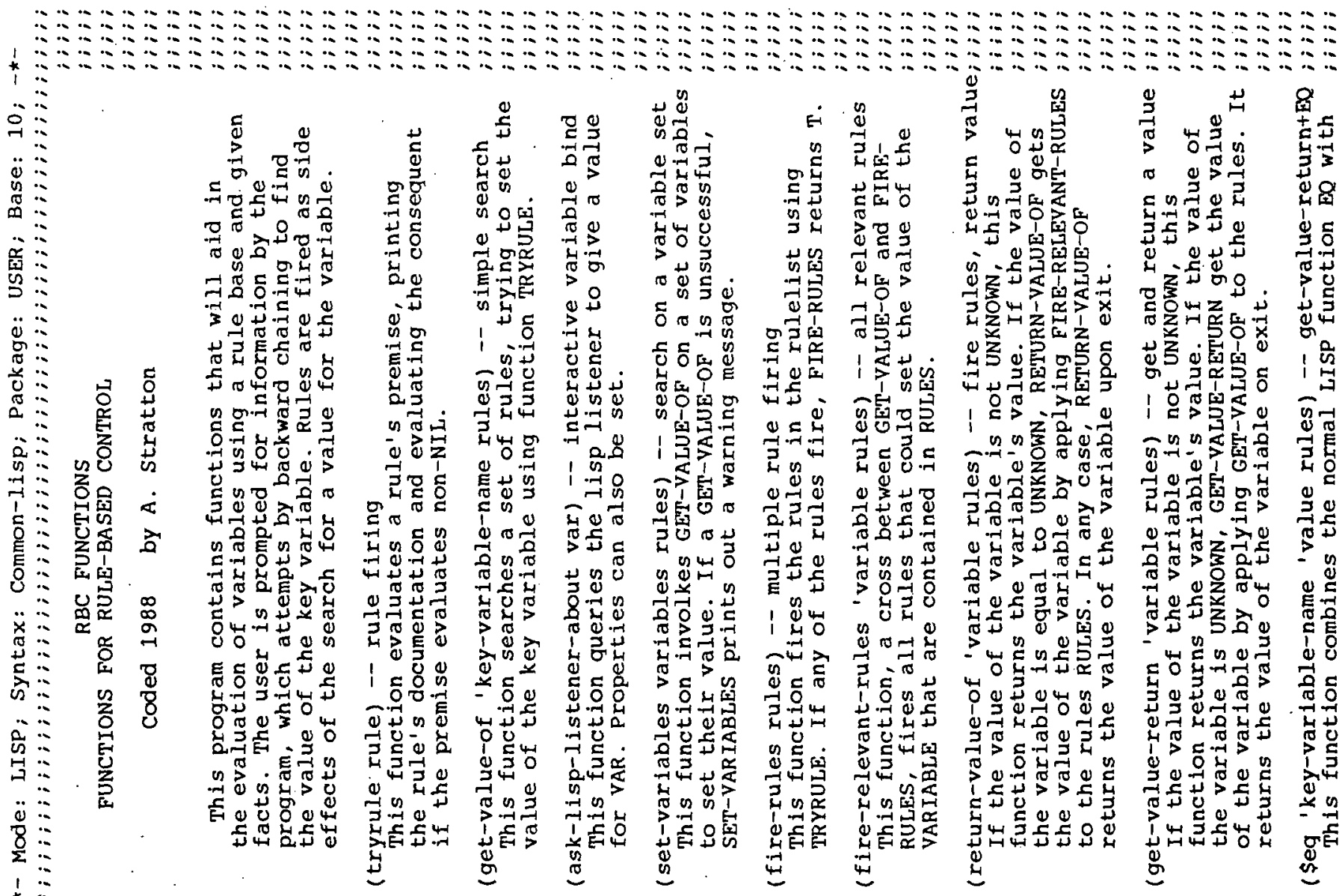




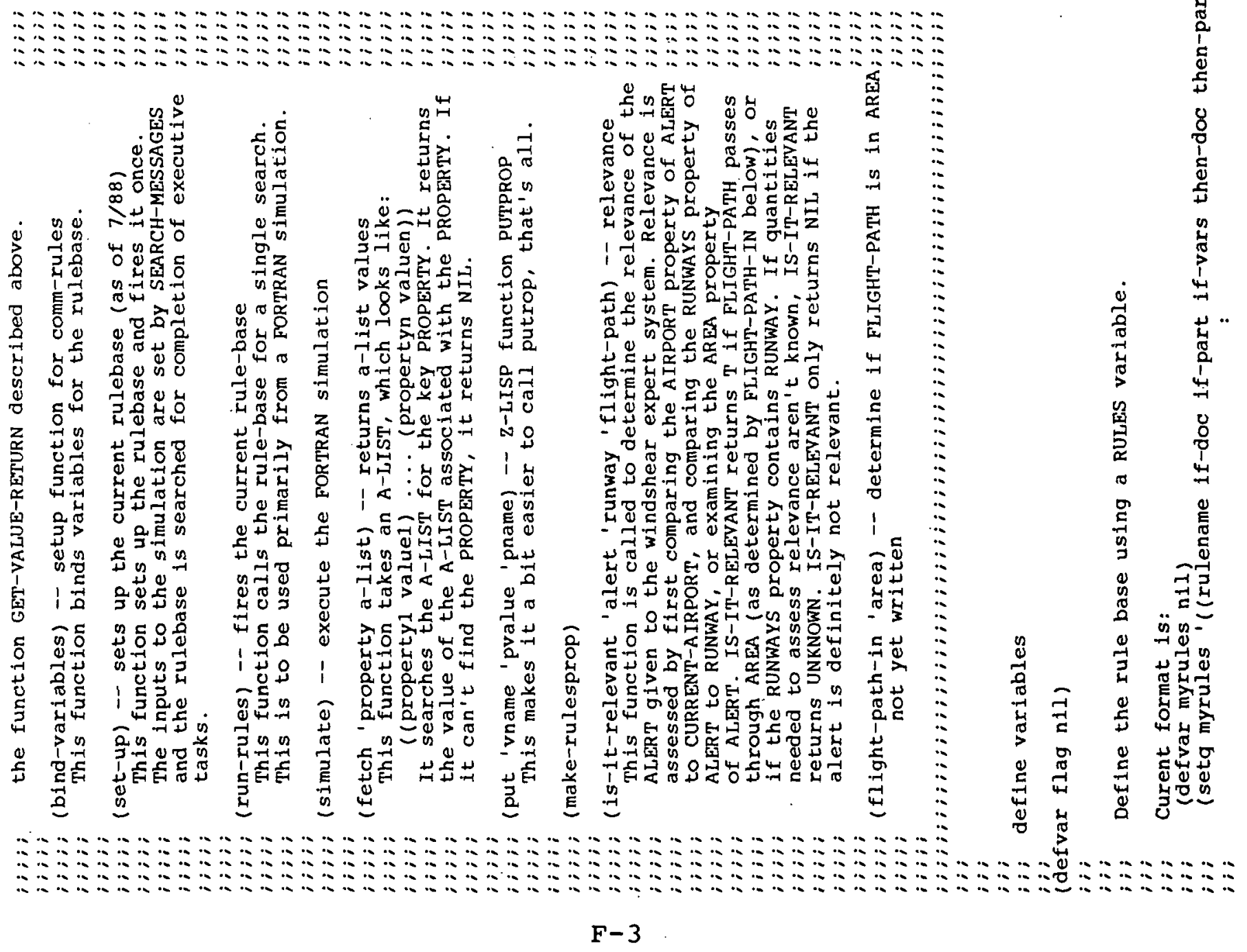




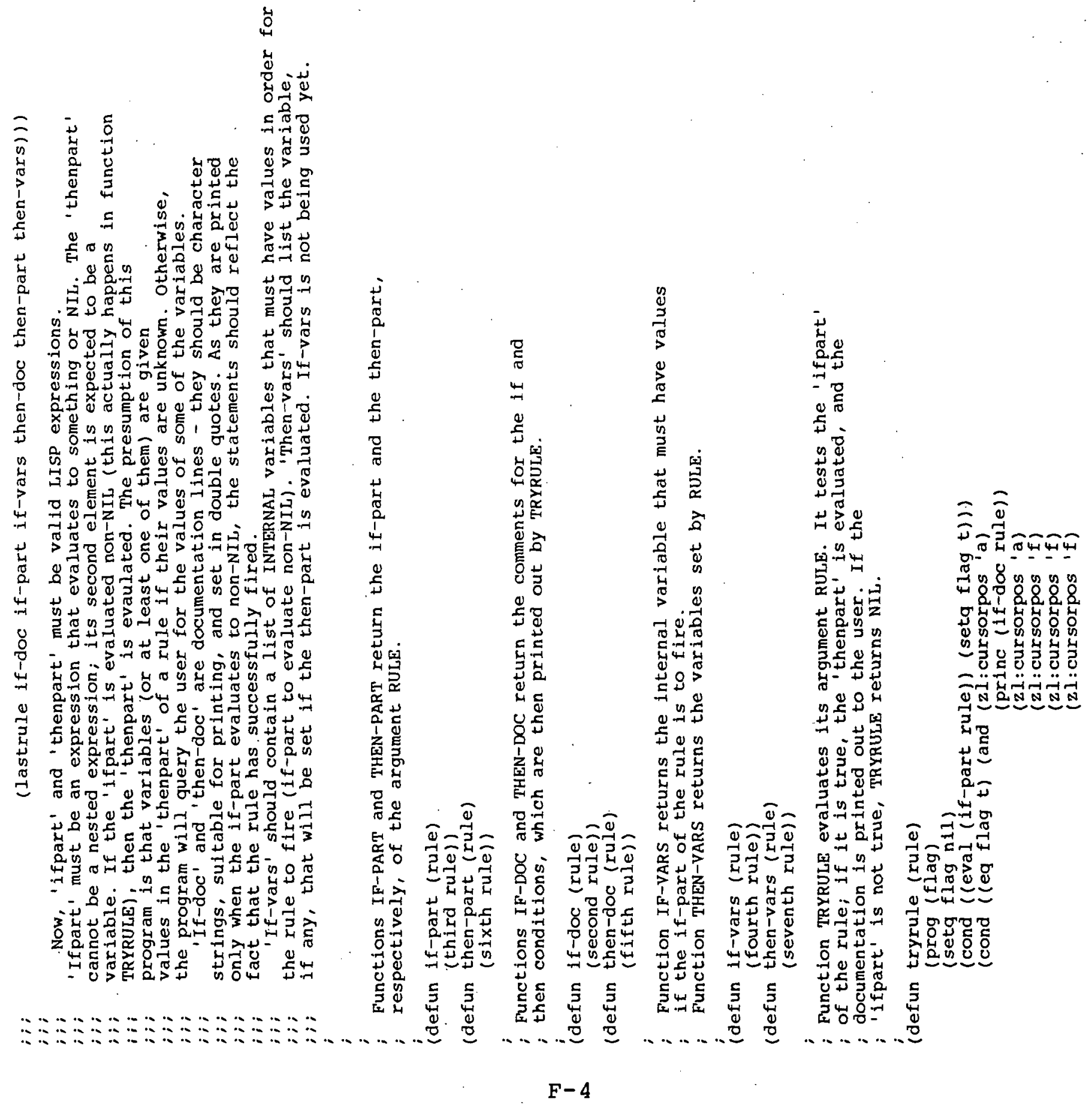




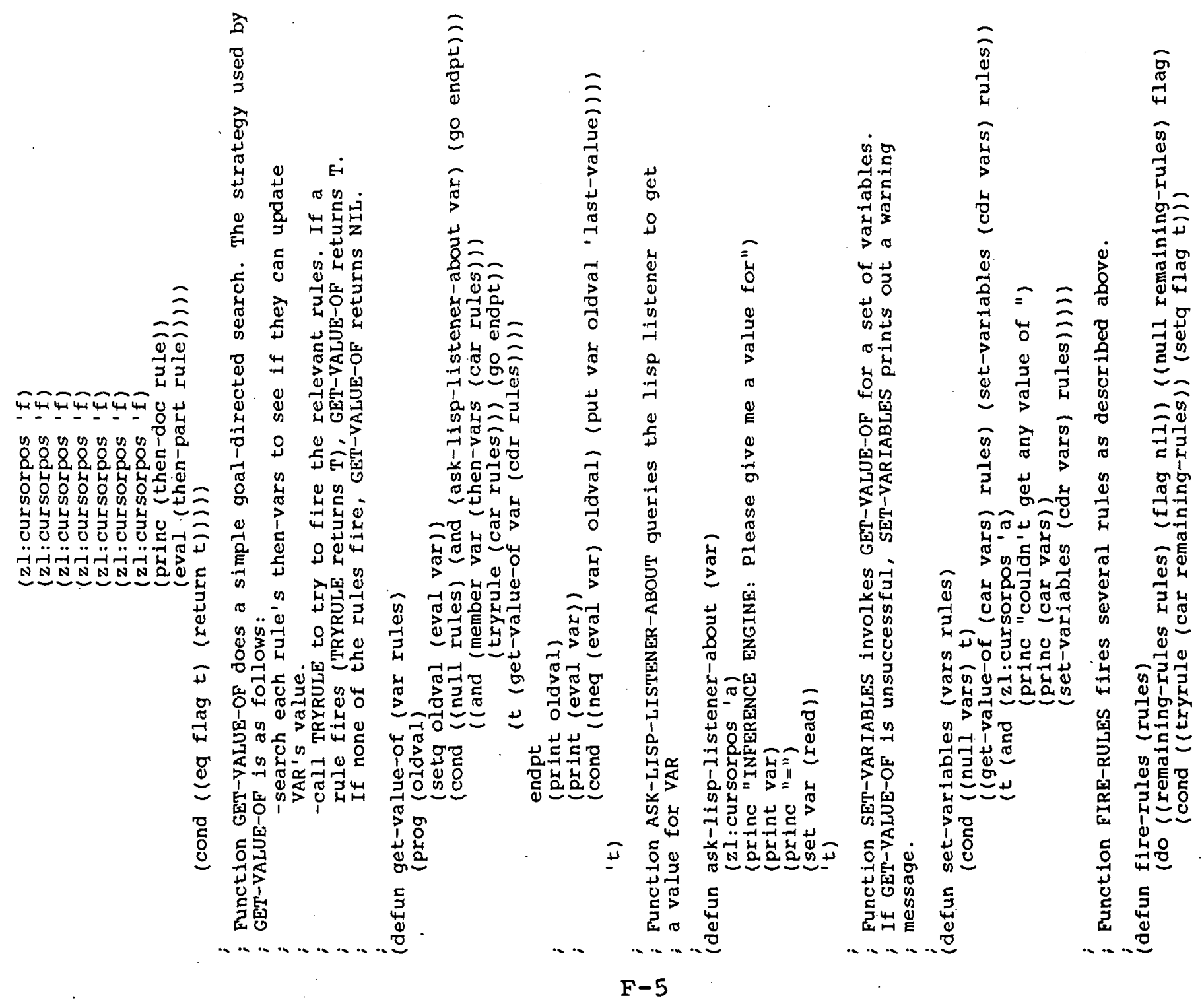




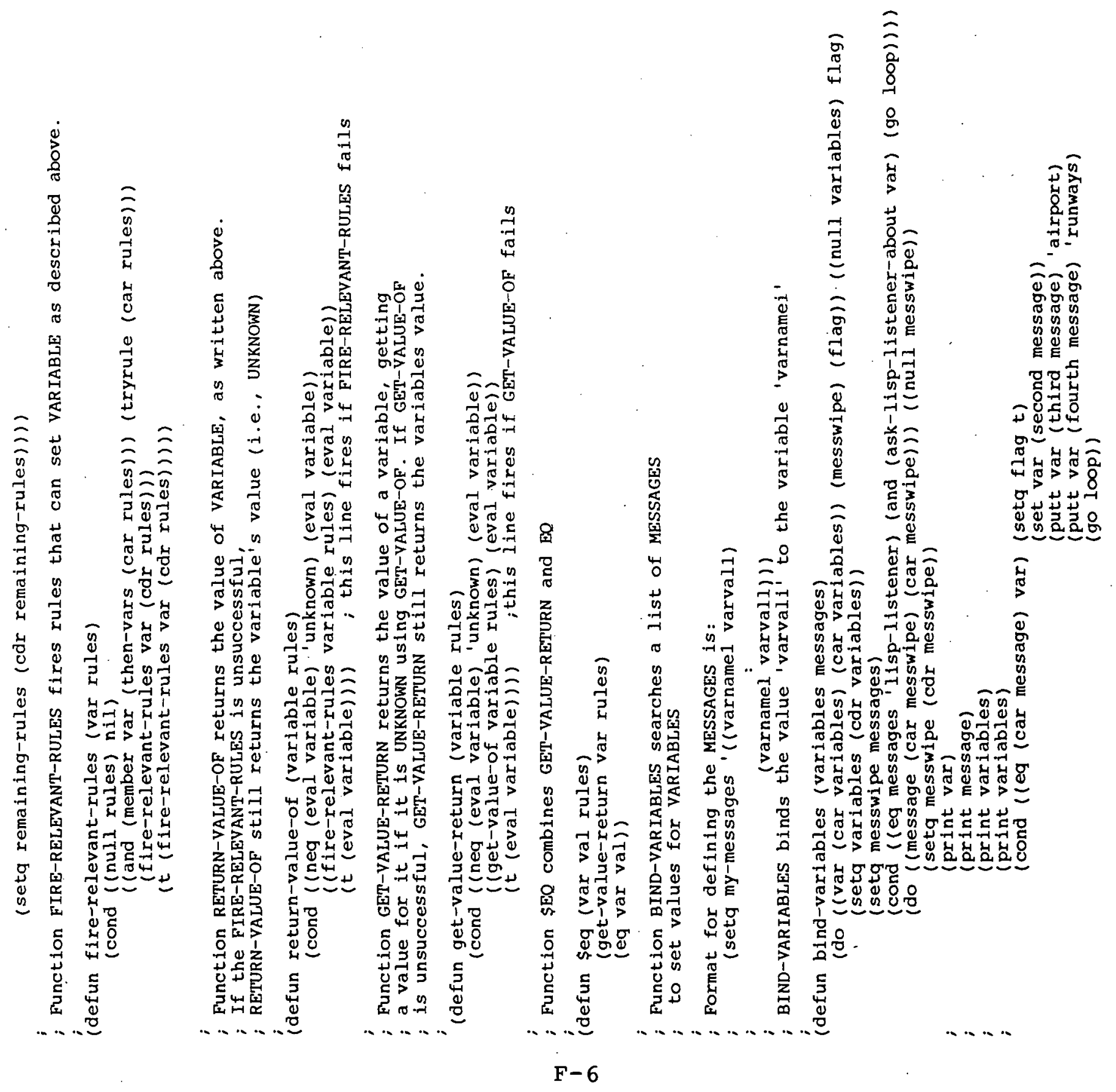


$\frac{1}{3}$

$\underset{2}{2}$

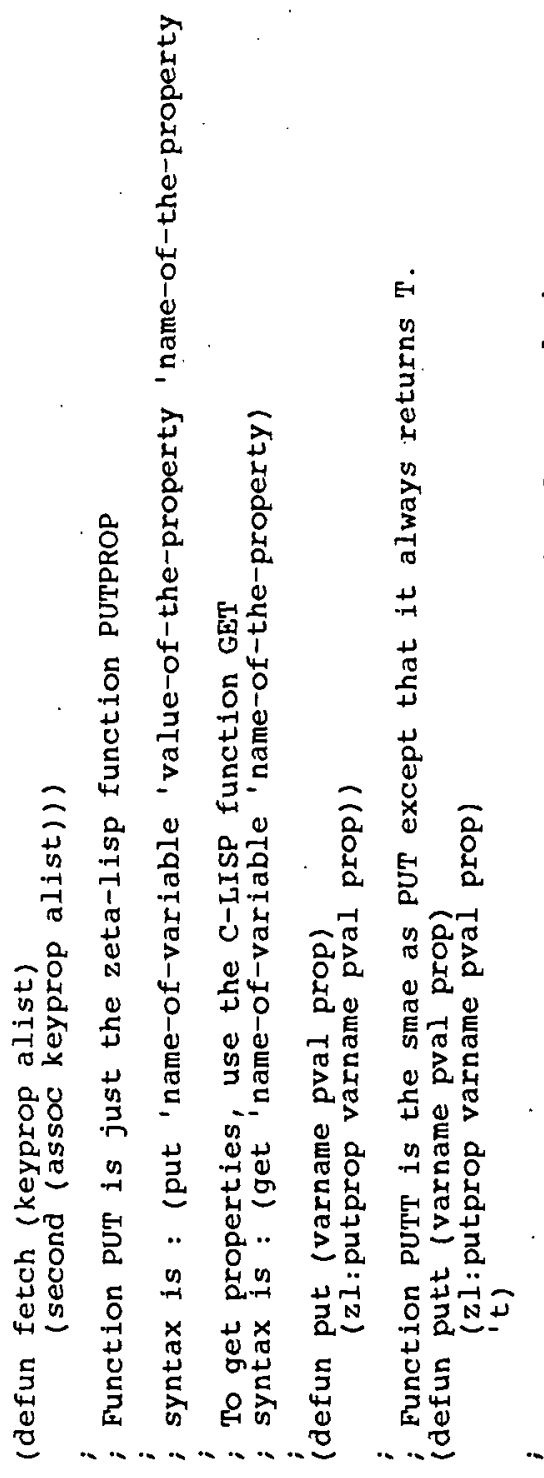

0
0
0
0
0
3
4
0
4
4
4
5
0
0
0
0
0
5
0
0
0

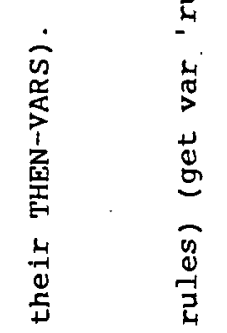

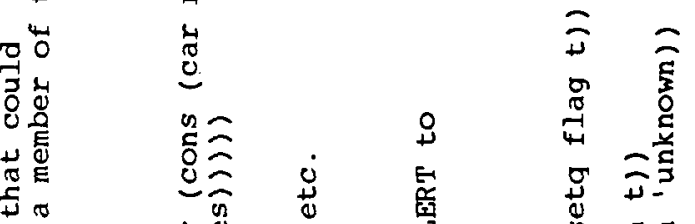

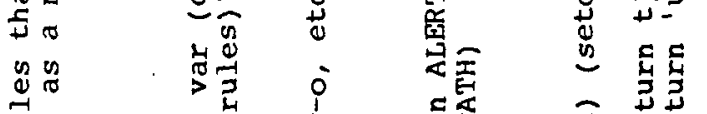

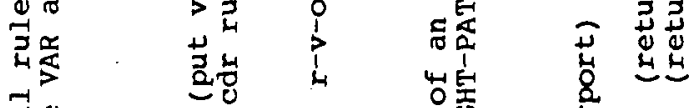

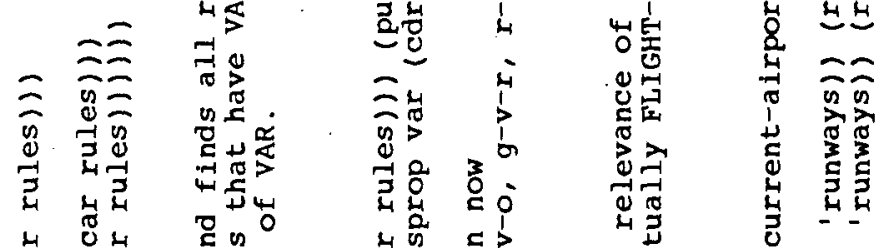

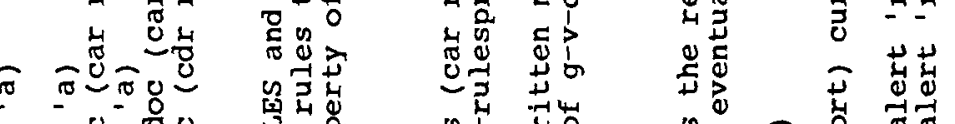

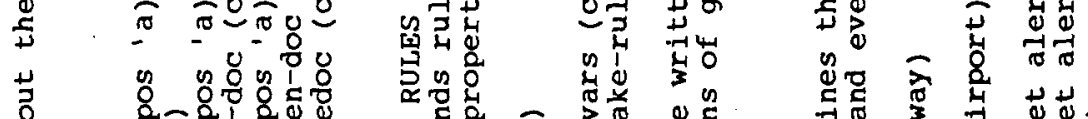

- 0

$\exists=0=0$

की

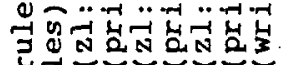

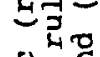

$\lim _{\substack{4 \\ 0}}$

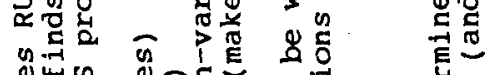

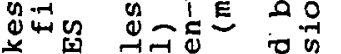

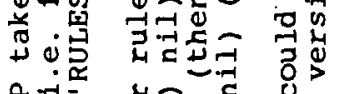

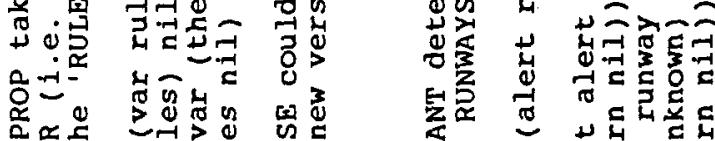

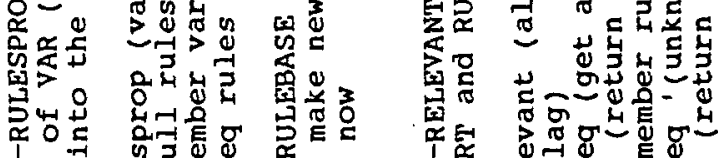

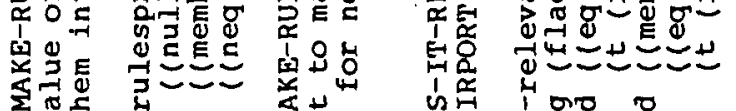

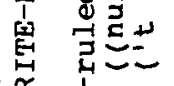

d

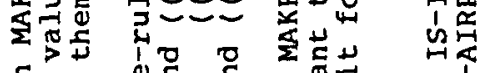

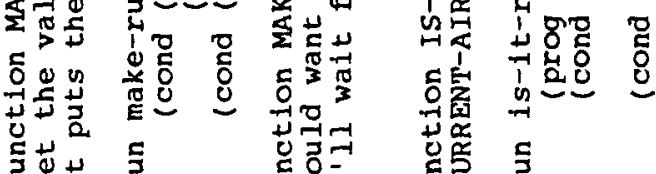

ᄃ

焉热出

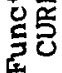

咅 


\section{Appendix G \\ Typical Screen Displays
from Wind Shear Expert System}

G-1 


\section{ORIGINAL PAGE IS \\ OF POOR QUALITY}

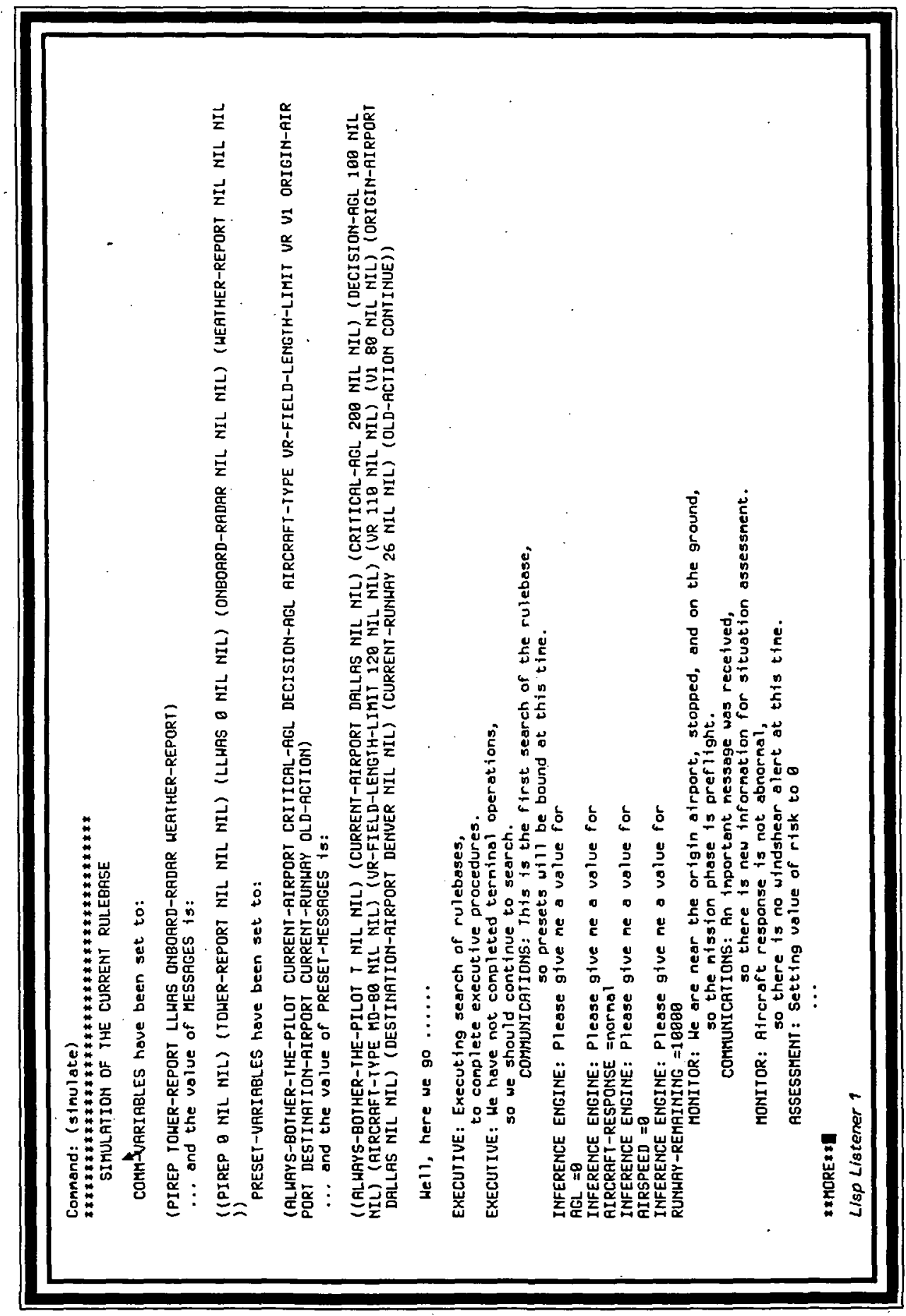


ORIGINAL PAGE IS

OF. POOR QUALITY

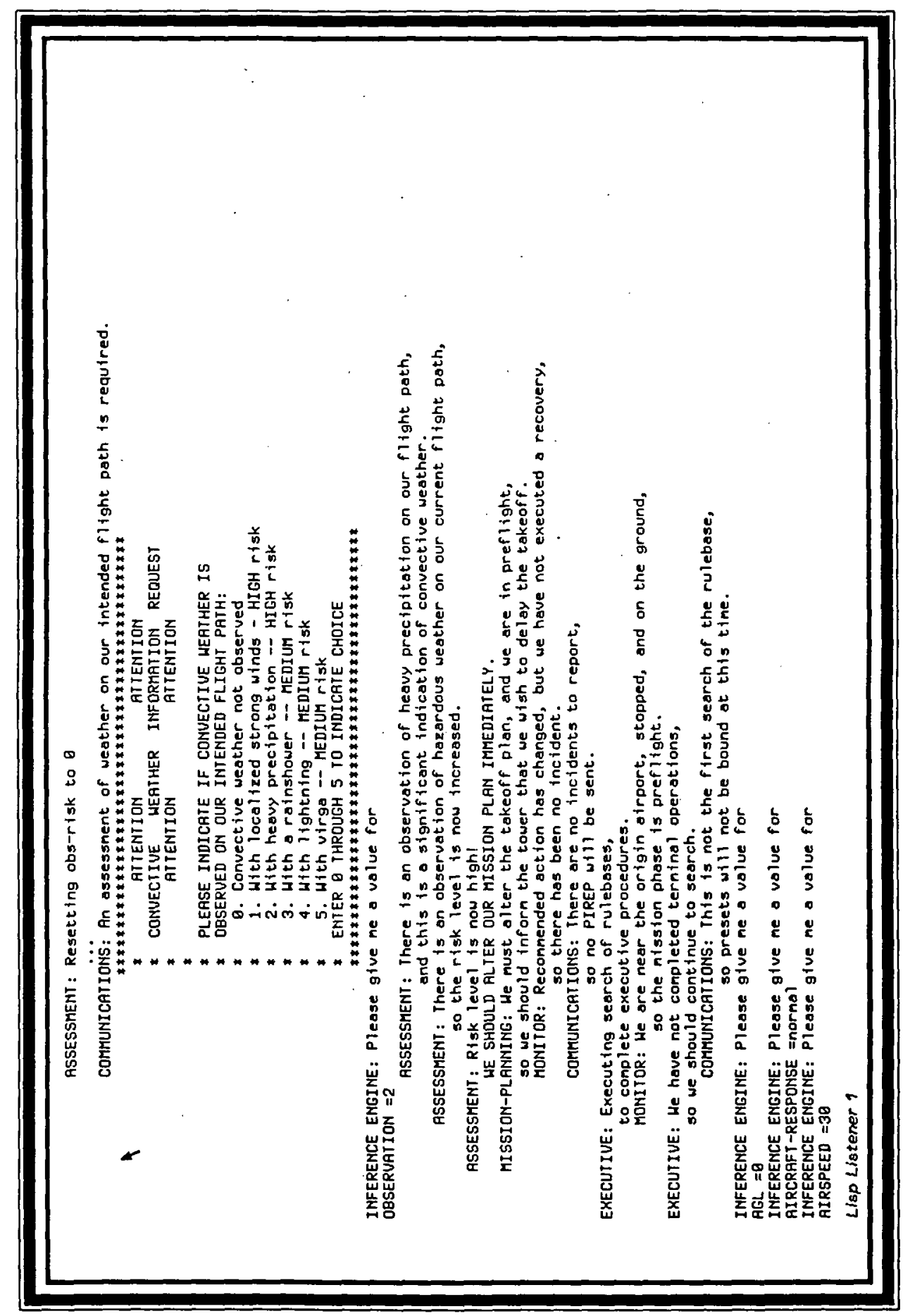




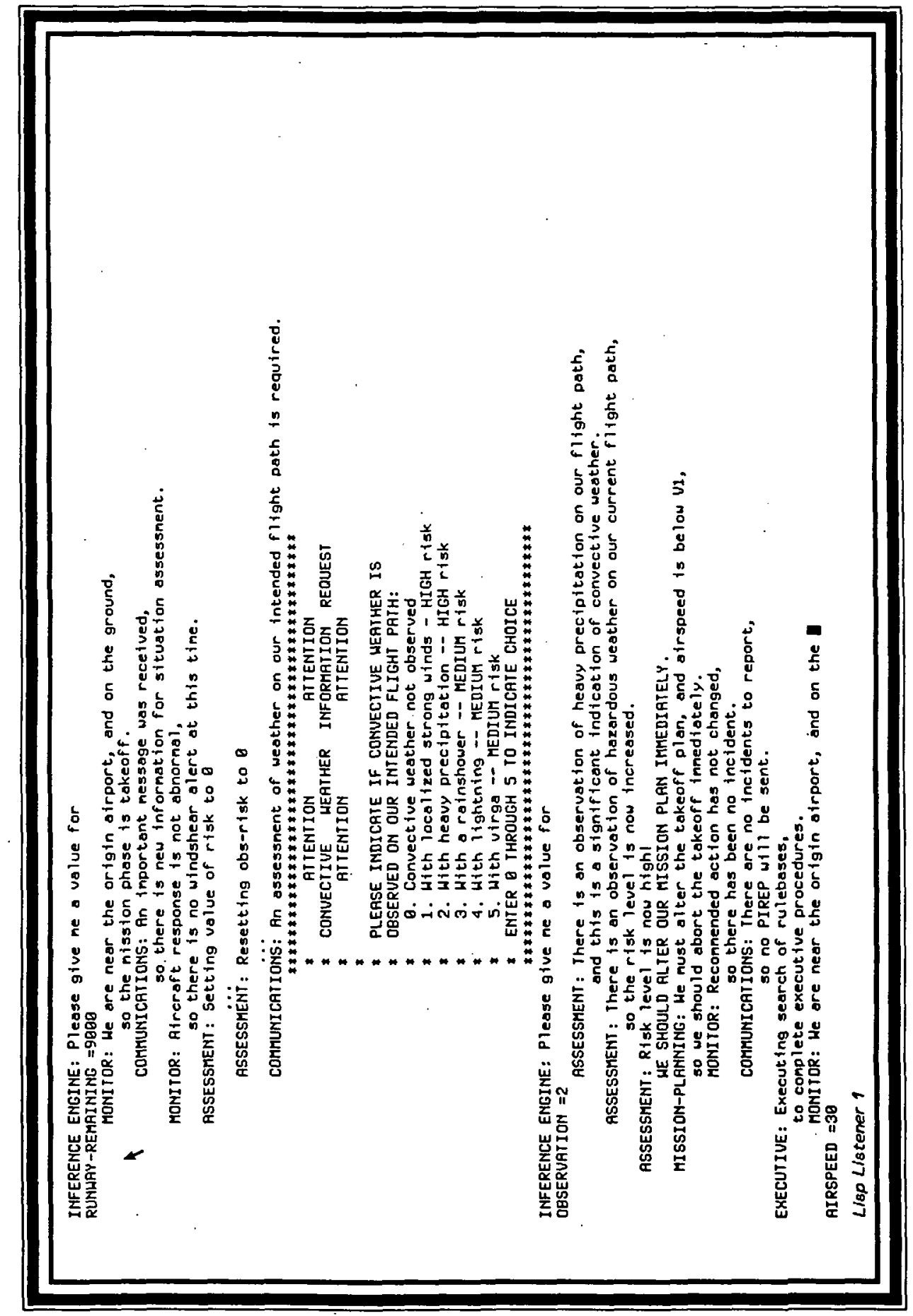




\section{Appendix $H$
Charts for Program Status Report, June, 1988 Charts for Program Status Report, June, 1988}




\title{
INTELLIGENT GUIDANCE AND CONTROL FOR WINDSHEAR ENCOUNTERS
}

\author{
INTRODUCTION \\ AND \\ PROGRESS REPORT
}

\author{
Alex Stratton \\ Princeton University \\ June 23-24, 1988
}


Overview

- Introduction

- Expert System Development

- Plans for Future Work

$\mathrm{H}-3$ 


\section{Motivation for the Study}

- Strong windshears, especially microbursts, can cause fatal accidents

- Pilot proficiency in coping with microburst windshear is difficult given rarity of phenomenon

- Proper decision making and control strategy can enhance the possibility of avoidance and survival

- F.A.A. Pilot Windshear Guide establishes such a strategy 


\section{Intelligent G\&C Research Goals}

- Implement the Pilot Windshear Guide pilot and crew model as a rule-based (intelligent) controller

- Investigate guidance and control concepts applied to windshear encounters

- Develop a rule-based pilot aid for windshear survival 


\section{Rule-based Pilot Aid - Schematic}

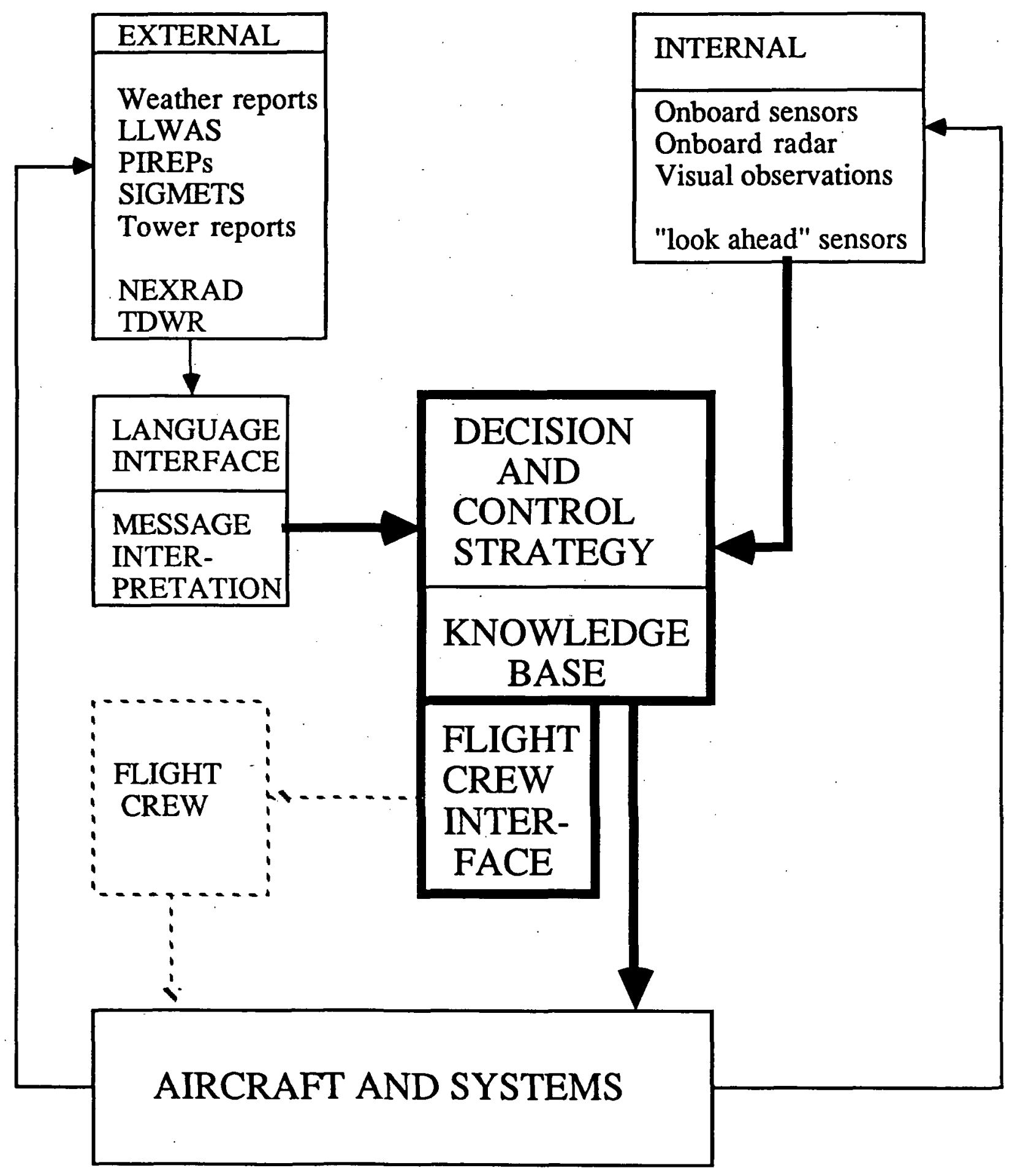




\section{Sources of Decision and Control Knowledge}

- Prior knowledge of windfield - Optimal control laws

\section{No knowledge of windfield - Strategy based on current knowledge in cockpit - Pilot Windshear Guide}




\section{Pilot Windshear Guide}

- Completed as part of the FAA's Windshear Training Aid

- Reference guide for flight crews detailing windshear avoidance, recognition, and recovery techniques

- Provides a model of pilot and crew decision-making that increases flight safety 


\section{Rule-based Systems for Control}

- Federated systems - Symbolic supervisory control Separate numeric processing

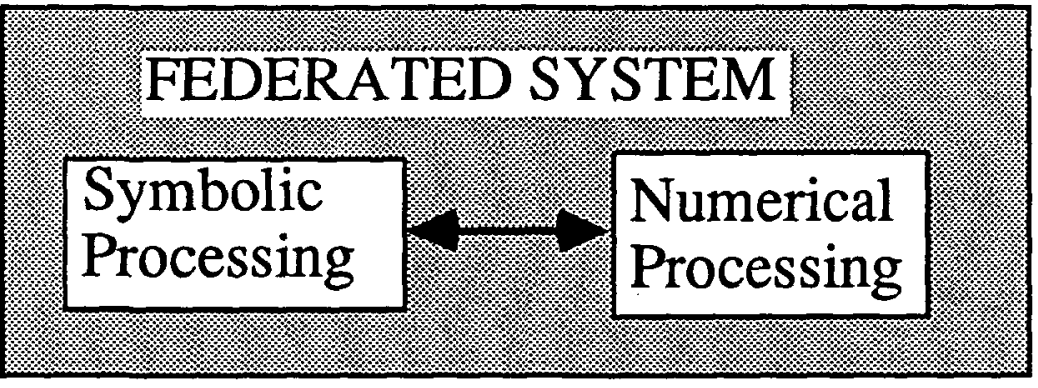

- Integrated systems - Combined symbolic and numeric control

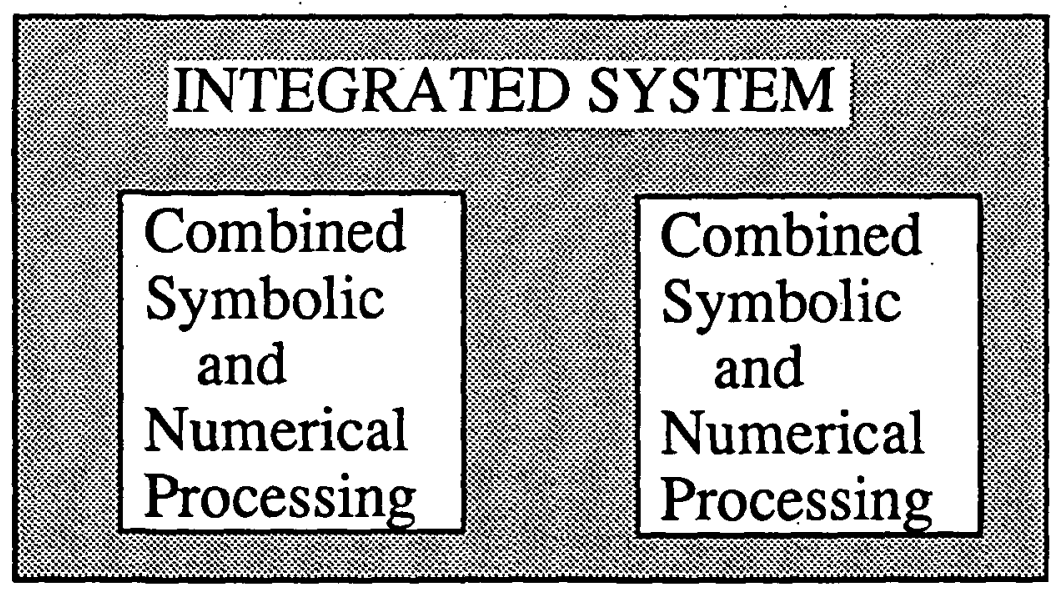




\section{Expert System Control Functions}

- MONITORING

- ASSESSMENT -- INTERPRETATION -- DIAGNOSIS

- PREDICTION -- PLANNING

- ACTION -- CONTROL 


\section{Expert System General Structure}

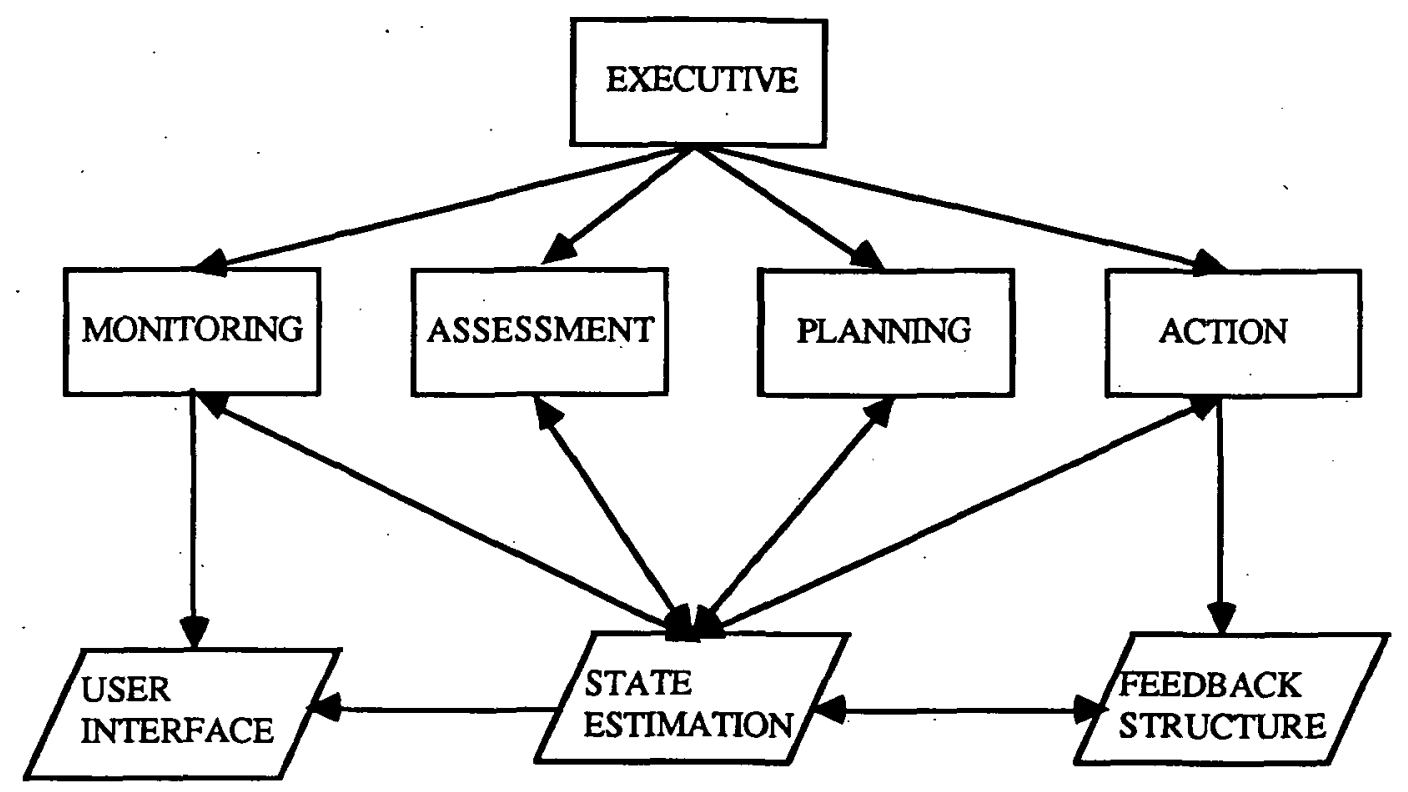




\section{Monitoring and Assessment}

- MONITORING -

Observe onboard sensors

Receive reports, alerts, warnings

Send reports, communicate with flight crew

- ASSESSMENT -

Determine the risk of continuing

Decide if the flight plan should be altered

Decide if windshear has been encountered 


\section{Planning and Action}

- PLANNING -

Delay or abort terminal operations if necessary Take additional safety precautions

- ACTION -

Select and implement guidance and control laws Send commands to control surfaces and throttle 


\section{Expert System Implementation}

- LISP -- Knowledge representation, symbolic processing

- FORTRAN -- Numerical procedures, algorithms

- Symbolics 3670 LISP Machine 


\section{Flow of Control Driven By Goal-Directed Search Procedure}

- Basic search procedure evaluates rules to set the value of a goal parameter

- Search procedure is often involked in the premise of a rule, resulting in backward-chaining

- More complicated search procedures are built with the basic procedure 


\section{Graphical Representation of Search}

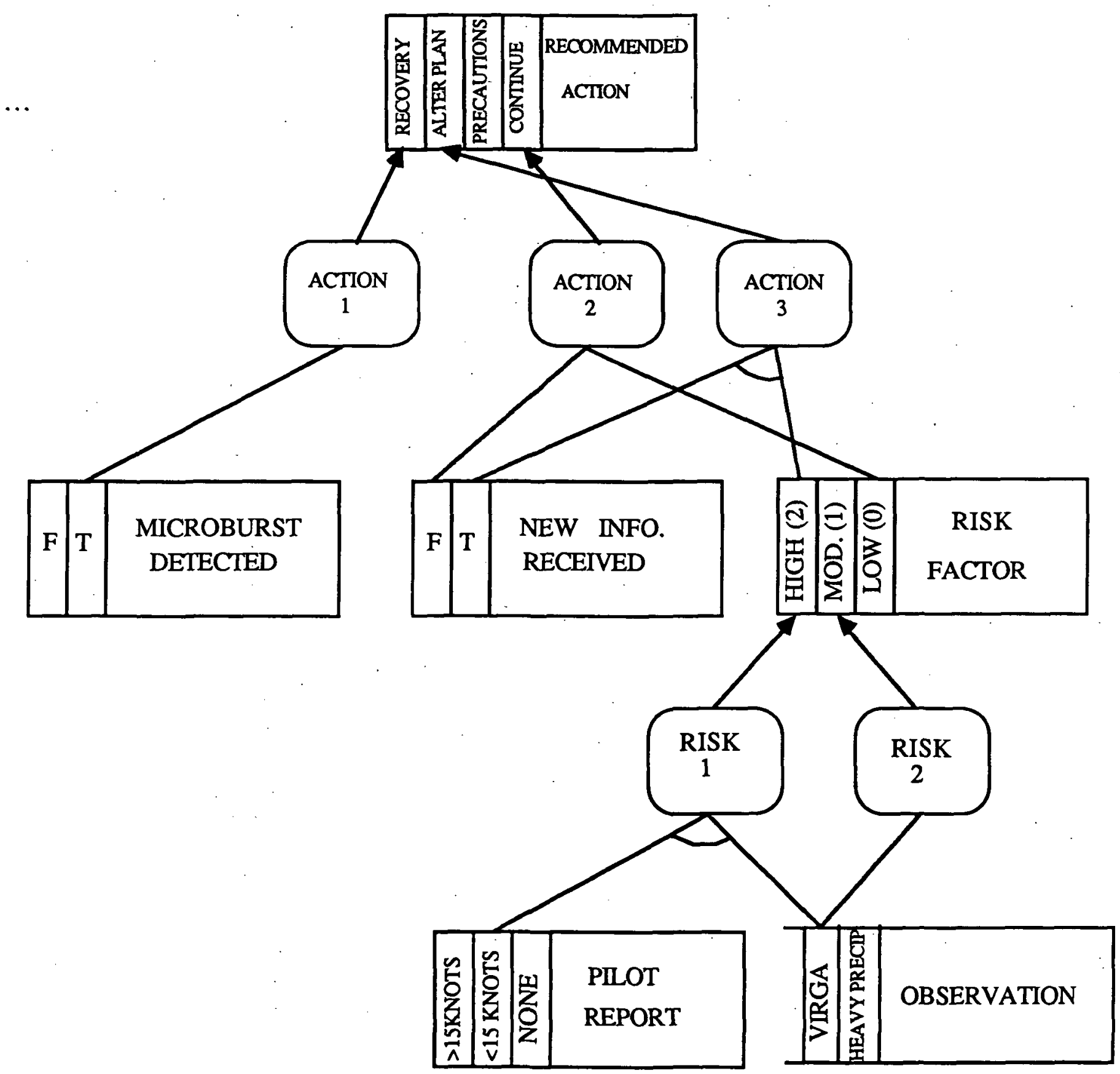




\section{Example of Knowledge Acquisition}

\section{PILOT WINDSHEAR GUIDE STATEMENT:}

"The choice of takeofff flap selection is dependant on the airplane type. The following should be considered unless limited by obstacle clearance and/or climb gradient:

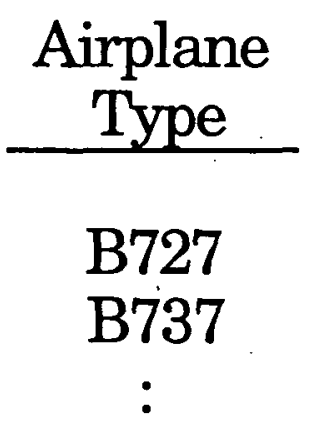

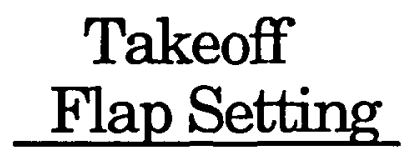

15

5 to 15

\section{PSEUDO-LISP TRANSLATION:}

IF recommended-action equals "take-precautions" AND aircraft-type equals "B727"

THEN set recommended-flap-setting to "15" 


\section{Expert System Detailed Structure}

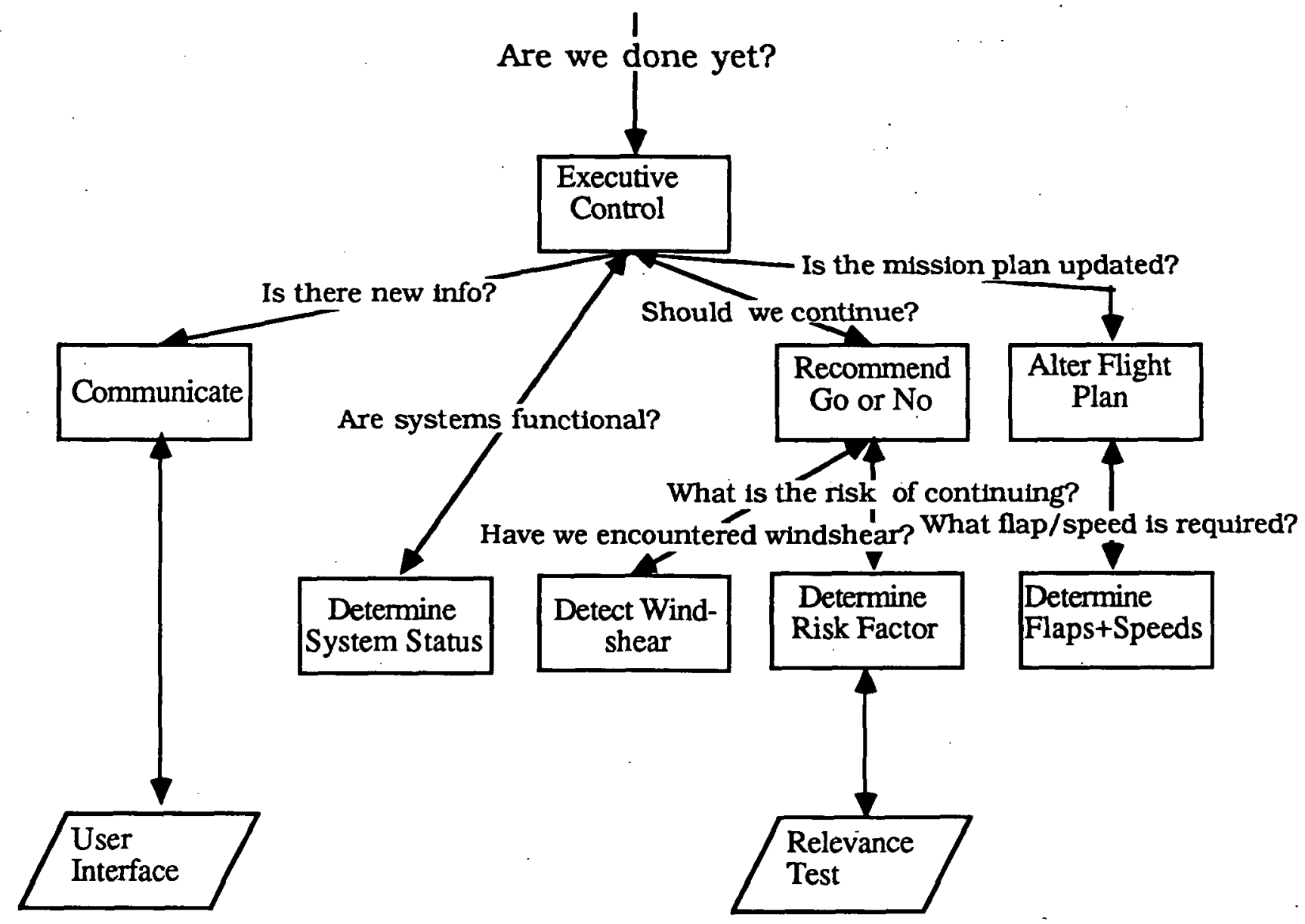




\section{Next Additions to Expert System}

FORTRAN procedures and simulation environment will be completed next

Low-level rules to interface FORTRAN procedures will complete knowledge base for Pilot Windshear Guide

Improved user interface will aid the understanding and further development of expert system pilot aid 


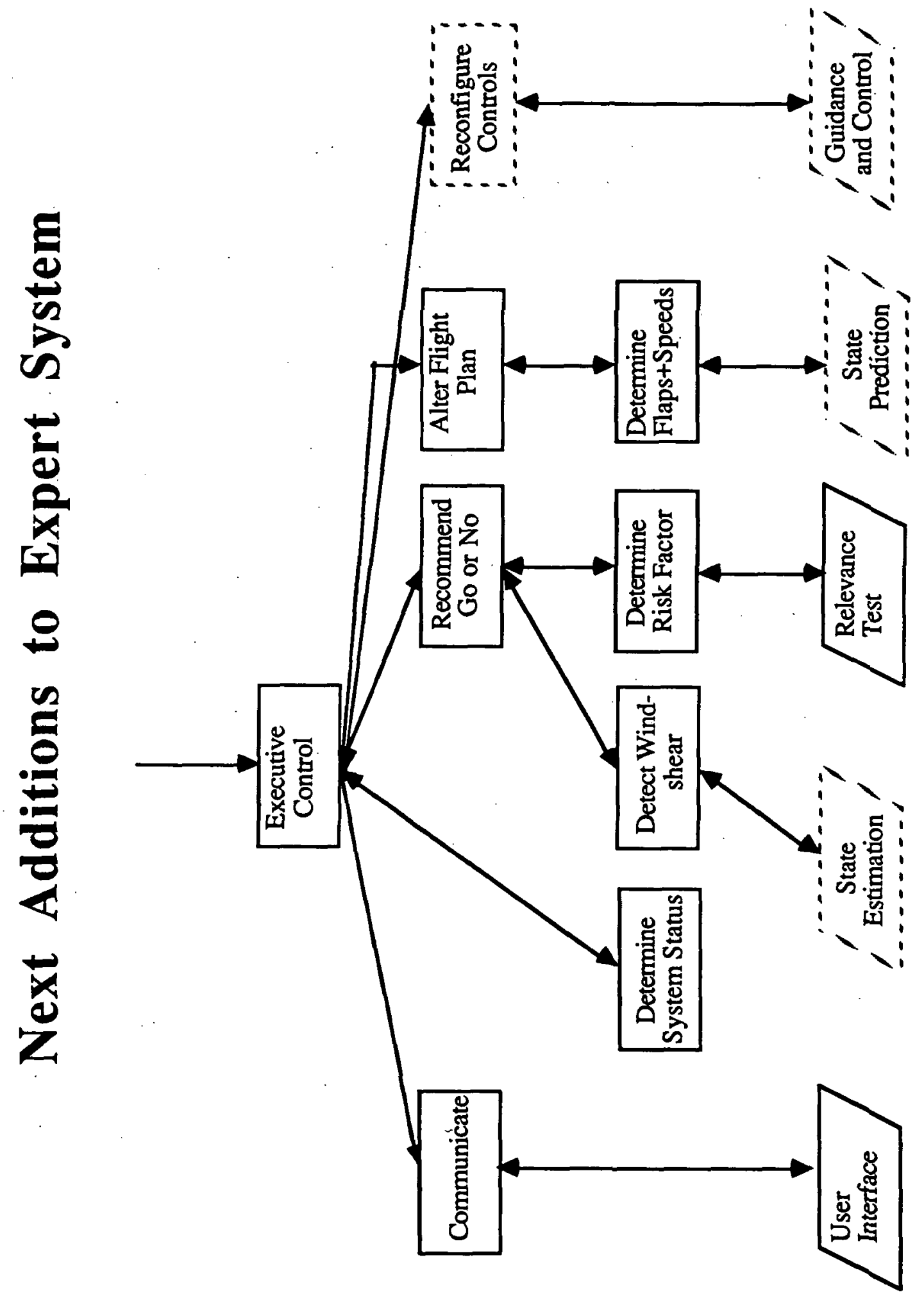




\section{Plans for Future Work}

- Consider impact of next-generation sensors and ATC

- Real-time implementation of RBC

- Consider pilot/RBC interface 